\title{
PRIMAL-DUAL BLOCK-PROXIMAL SPLITTING FOR A CLASS OF NON-CONVEX PROBLEMS*
}

\author{
STANISLAV MAZURENKO ${ }^{\dagger}$, JYRKI JAUHIAINEN ${ }^{\ddagger}$, AND TUOMO VALKONEN ${ }^{\S}$
}

\begin{abstract}
We develop block structure-adapted primal-dual algorithms for non-convex non-smooth optimisation problems, whose objectives can be written as compositions $G(x)+F(K(x))$ of non-smooth block-separable convex functions $G$ and $F$ with a nonlinear Lipschitz-differentiable operator $K$. Our methods are refinements of the nonlinear primal-dual proximal splitting method for such problems without the block structure, which itself is based on the primal-dual proximal splitting method of Chambolle and Pock for convex problems. We propose individual step length parameters and acceleration rules for each of the primal and dual blocks of the problem. This allows them to convergence faster by adapting to the structure of the problem. For the squared distance of the iterates to a critical point, we show local $O(1 / N), O\left(1 / N^{2}\right)$, and linear rates under varying conditions and choices of the step length parameters. Finally, we demonstrate the performance of the methods for the practical inverse problems of diffusion tensor imaging and electrical impedance tomography.
\end{abstract}

Key words. primal-dual algorithms, convex optimization, non-smooth optimization, step length

AMS subject classifications. 49M29, 65K10, 90C30

1. Introduction. We want to solve in Hilbert spaces $X$ and $Y$ the problem

$$
\min _{x \in X} G(x)+F(K(x))
$$

where $G: X \rightarrow \overline{\mathbb{R}}$ and $F: Y \rightarrow \overline{\mathbb{R}}$ are convex, proper, and lower semicontinuous functions but $K \in C^{1}(X ; Y)$ is a possibly nonlinear operator. The linear case has been considered frequently in the literature, while in our earlier work $[9,11,33]$ we have developed first-order primal-dual methods for the generally non-convex problem with a nonlinear $K$. We refer to [36] for a simplified overview of such methods. In the present work, still with a nonlinear $K$, we consider problems of the more specific form

$$
\min _{x \in X} \sum_{j=1}^{m} G_{j}\left(P_{j} x\right)+\sum_{\ell=1}^{n} F_{\ell}\left(Q_{\ell} K(x)\right)
$$

where, for all $j=1, \ldots, m$ and $\ell=1, \ldots, n$, the functions $G_{j}: X \rightarrow \overline{\mathbb{R}}$ and $F_{\ell}: Y \rightarrow \overline{\mathbb{R}}$ are convex, proper, and lower semicontinuous, and $P_{1}, \ldots, P_{m} \in \mathbb{L}(X ; X)$ as well as $Q_{1}, \ldots, Q_{n} \in \mathbb{L}(Y ; Y)$ are mutually orthogonal families of linear projection operators. In other words, $G$ and $F$ are block-separable. More specifically, we develop spatially adaptive and block-stochastic optimisation methods for the solution of (P).

As observed in [35] for linear $K$, the adaptation of step lengths to individual blocks $j$ and $\ell$ can speed up the convergence of optimisation methods due to blockwise Lipschitz or strong convexity factors being better suited than a global factor. Moreover, as now extensively studied, randomly sampling the blocks to be updated in each step can also improve convergence for very large-scale problems, in part due to the spatial adaptation and in part due to being

* Received November 14, 2019. Accepted July 17, 2020. Published online on September 24, 2020. Recommended by Fiorella Sgallari.

${ }^{\dagger}$ Loschmidt Laboratories, Masaryk University, Brno, Czechia (stan . mazurenko@gmail . com), ORCID: 0000-0003-3659-4819.

$\ddagger$ University of Eastern Finland, Kuopio, Finland (jyrki . jauhiainen@uef . fi), ORCID: 0000-0001-6711-6997.

$\S$ ModeMat, Escuela Politécnica Nacional, Quito, Ecuador and Department of Mathematics and Statistics, University of Helsinki, Finland (tuomo . valkonen@iki.fi), orCID: 0000-0001-6683-3572. 
able to avoid communication in a cluster implementation of the algorithm. For more on stochastic block coordinate descent-type methods, we refer to the review [42] and, among others, the original articles $[2,13,16,23,25,28,29,31,46]$ on forward-backward type methods, $[4,6,8,12,15,32,35,45]$ on primal-dual methods, and [26, 27] on second-order methods, all in the convex case. For the non-convex case we point to [43, 44]. Compared to the latter, we work in the primal-dual setting and aim for spatial adaptation also in the deterministic setting. We also aim to prove convergence rates.

Several works consider, instead of a random selection of blocks, a random selection of terms of a sum of functions. In the non-convex case, recent mathematical works in this area include [14, 22], aside from more applied works in the area of neural networks. In our block-stochastic approach, for non-convex $C^{1}$-functions $J_{\ell}(\ell=1, \ldots, n)$, we can write with $K(x):=\left(J_{1}(x), \ldots, J_{n}(x)\right)$ and $F(z):=\sum_{\ell=1}^{n} z_{\ell}$,

$$
\min _{x} G(x)+\sum_{\ell=1}^{n} J_{\ell}(x)=\min _{x} G(x)+F(K(x)) .
$$

To start describing our approach, using the conjugates $F_{\ell}^{*}$ of the convex, proper, lower semicontinuous functions $F_{\ell}$, we reformulate $(\mathrm{P})$ as the minmax problem

$$
\min _{x \in X} \max _{y \in Y} \sum_{j=1}^{m} G_{j}\left(P_{j} x\right)+\langle K(x), y\rangle-\sum_{\ell=1}^{n} F_{\ell}^{*}\left(Q_{\ell} y\right) .
$$

If $K$ is linear and the number of blocks is $n=m=1$, then a popular algorithm for solving this formulation is the primal-dual proximal splitting (PDPS) of Chambolle and Pock [7]. It consists of alternating proximal steps with respect to the dual and primal variables with the other variable fixed and an over-relaxation step that ensures convergence. Its extension to nonlinear $K$ (but still without blockwise structure) is the iteration $[9,33]$

$$
\left\{\begin{array}{l}
x^{i+1}:=\operatorname{prox}_{\tau_{i} G}\left(x^{i}-\tau_{i} \nabla K\left(x^{i}\right)^{*} y^{i}\right) \\
\bar{x}^{i+1}:=x^{i+1}+\omega_{i}\left(x^{i+1}-x^{i}\right) \\
y^{i+1}:=\operatorname{prox}_{\sigma_{i+1} F^{*}}\left(y^{i}+\sigma_{i+1} K\left(\bar{x}^{i+1}\right)\right)
\end{array}\right.
$$

for some step length and over-relaxation parameters $\tau_{i}, \sigma_{i+1}, \omega_{i}$ and the proximal operator $\operatorname{prox}_{\tau_{i} G}(x):=\left(I+\tau_{i} \partial G\right)^{-1}(x)$. Our purpose in this work is to randomise and adapt the method to the multi-block structure of (S): firstly, in each step, we will only update random subsets of either or both primal and dual blocks, and, secondly, even when we deterministically update every block in each step, we adapt the step lengths to the local structure of the problem in each block.

We organise our work as follows: first, in Section 2, we introduce the general notation, concepts, and the rough structure of the algorithm. In Section 3 we start the convergence proof by deriving several technical estimates. In Section 4 we then use these estimates to derive convergence rates of more specific algorithms when only the primal updates are randomised. Likewise, in Section 5 we study the case when only the dual updates are randomised. We finish our work in Section 6 with numerical experience in diffusion tensor imaging (DTI) and electrical impedance tomography (EIT).

2. Notation, rough algorithm, and its testing. Throughout this paper, we denote by $\mathbb{L}(X ; Y)$ the space of bounded linear operators between Hilbert spaces $X$ and $Y, I$ is the identity operator, and $\left\langle x, x^{\prime}\right\rangle$ is the inner product in the corresponding space. We write $\mathcal{P} A$ for the power set of a set $A$ and $\chi_{A}(a)$ for the indicator function that equals 1 if $a \in A$ and 0 


\section{ETNA}

Kent State University and

Johann Radon Institute (RICAM)

otherwise. We set $\left\langle x, x^{\prime}\right\rangle_{T}:=\left\langle T x, x^{\prime}\right\rangle$ and $\|x\|_{T}:=\sqrt{\langle x, x\rangle_{T}}$, where in the latter we require $T \geq 0$. For $T, S \in \mathbb{L}(X ; Y)$, the inequality $T \geq S$ means that $T-S$ is positive semidefinite. If $H$ is a set-valued operator $X \rightrightarrows X$, then inequalities such as $\left\langle H(x), x^{\prime}\right\rangle \geq 0$ mean that $\left\langle w, x^{\prime}\right\rangle \geq 0$ for every $w \in H(x)$.

We write $(\Omega, \mathcal{O}, \mathbb{P})$ for the probability space consisting of a sample set $\Omega$, a $\sigma$-algebra $\mathcal{O}$ on $\Omega$, and a probability measure $\mathbb{P}$. We write $\mathcal{R}(\mathcal{O} ; V)$ for the space of $V$-valued $\mathcal{O}$-measurable random variables. $\mathcal{R}(\mathcal{O} ; U \rightrightarrows U)$ is therefore the space of $\mathcal{O}$-measurable random variables, whose values are set-valued operators $U \rightrightarrows U$. Due to the iterative nature of optimisation algorithms, we introduce a sequence of $\sigma$-algebras $\left\{\mathcal{O}_{i}\right\}_{i \in \mathbb{N}}$ such that $\mathcal{O}_{i} \subseteq \mathcal{O}_{i+1}$ and $\mathcal{O}_{i} \subseteq \mathcal{O}$ for any $i \in \mathbb{N}$. We use $\mathcal{O}_{i}$ to collect all the information available before the $(i+1)$ st iteration. We write $\mathbb{E}_{i}[\cdot]:=\mathbb{E}\left[\cdot \mid \mathcal{O}_{i}\right]$ for the corresponding conditional expectation.

Many conditions that we impose in the following sections only apply to the subspace on which the operator $K$ from the introduction acts nonlinearly. Correspondingly, we introduce

$$
Y_{\mathrm{L}}:=\{y \in Y \mid \text { the map } x \mapsto\langle y, K(x)\rangle \text { is linear }\} \quad \text { and } \quad Y_{\mathrm{NL}}:=Y_{\mathrm{L}}^{\perp} \text {, }
$$

as well as the orthogonal projection $P_{\mathrm{NL}}$ to $Y_{\mathrm{NL}}$. See Section 6 for how such subspaces practically come about in applications. We also use the short-hand notation

$$
x_{j}:=P_{j} x \quad \text { and } \quad y_{\ell}:=Q_{\ell} y .
$$

2.1. Abstract structure of the algorithm. We generally use the symbol $x$ for primal variables (elements of $X$ ) and the symbol $y$ for dual variables (elements of $Y$ ). We group these variables together into $u=(x, y) \in X \times Y$. This applies to indexed variables, $u^{i}:=\left(x^{i}, y^{i}\right)$, critical points $\widehat{u}=(\widehat{x}, \widehat{y})$, etc., without an explicit introduction of the primal and dual components in each case. We define the set-valued operator $H: X \times Y \rightrightarrows X \times Y$ for $u=(x, y)$ as

$$
\begin{aligned}
& H(u):=\left[\begin{array}{c}
\partial G(x)+\nabla K(x)^{*} y \\
\partial F^{*}(y)-K(x)
\end{array}\right] \\
& \text { with } G(x):=\sum_{j=1}^{m} G_{j}\left(P_{j} x\right) \text { and } F^{*}(y):=\sum_{\ell=1}^{n} F_{\ell}^{*}\left(Q_{\ell} y\right) .
\end{aligned}
$$

Then $0 \in H(\widehat{u})$ encodes the critical point conditions for $(\mathrm{S})$. These will also become the first-order necessary optimality conditions under a constraint qualification, e.g., when $G$ is $C^{1}$ and either the null space of $\nabla K(x)^{*}$ is trivial or $\operatorname{dom} F=X$ [30, Example 10.8].

Following the "testing" approach to the convergence analysis from [34], we introduce the primal-dual step length, testing, and preconditioning operators

$$
\begin{aligned}
W_{i+1} & :=\left[\begin{array}{cc}
T_{i} & 0 \\
0 & \Sigma_{i+1}
\end{array}\right], \quad Z_{i+1}:=\left[\begin{array}{cc}
\Phi_{i} & 0 \\
0 & \Psi_{i+1}
\end{array}\right], \quad \text { and } \\
M_{i+1} & :=\left[\begin{array}{cc}
I & -\Phi_{i}^{-1} \Lambda_{i}^{*} \\
-\Psi_{i+1}^{-1} \Lambda_{i} & I
\end{array}\right] .
\end{aligned}
$$

Here, $T_{i}, \Phi_{i}$ and $\Sigma_{i+1}, \Psi_{i+1}$ are the respective primal and dual step length and testing operators, and $\Lambda_{i}$ is a term that we will develop to suitably decouple the updates of the primal and dual variables. In the deterministic case, $T_{i}, \Phi_{i} \in \mathbb{L}(X ; X)$, and $\Sigma_{i+1}, \Psi_{i+1} \in \mathbb{L}(Y ; Y)$, as well as $\Lambda_{i} \in \mathbb{L}(X ; Y)$. We assume $\Phi_{i}$ and $\Psi_{i+1}$ to be self-adjoint. This implies that also $Z_{i+1} M_{i+1}$ is self-adjoint. For the stochastic setting we will impose our formal assumptions later in (3.14). We will in particular require the tests $\Phi_{i}$ and $\Psi_{i+1}$ to be already known before 


\section{ETNA}

Kent State University and

Johann Radon Institute (RICAM)

the start of the $i$ th iteration (calculating $u^{i}$ ), whereas the step lengths themselves will have to be known before the $(i+1)$ st iteration (calculating $u^{i+1}$ ).

Finally, we write our proposed algorithm in the implicit form

$$
0 \in W_{i+1} \widetilde{H}_{i+1}\left(u^{i+1}\right)+M_{i+1}\left(u^{i+1}-u^{i}\right)
$$

for

$$
\begin{aligned}
\widetilde{H}_{i+1}\left(u^{i+1}\right):= & H\left(u^{i+1}\right) \\
& +\left[K\left(x^{i+1}\right)-K\left(x^{i+1}+\Omega^{i}\left(x^{i+1}-x^{i}\right)\right)+\nabla K\left(x^{i}\right) \Omega^{i}\left(x^{i+1}-x^{i}\right)\right]
\end{aligned}
$$

and some over-relaxation operator $\Omega_{i}$, which in the deterministic setting is in $\mathbb{L}(X ; X)$. Here $\widetilde{H}_{i+1}(u)$ is a partial linearization of $H(u)$ similar to [33]. It simplifies to $H(u)$ for a linear $K$. In the following, by specifying the testing, step length, preconditioning, and over-relaxation operator, we develop more explicit methods from this implicit formulation, which itself is more amenable to a convergence analysis.

2.2. Testing for convergence. The proximal point method iteratively solves $u^{i+1}$ from

$$
0 \in H\left(u^{i+1}\right)+\tau^{-1}\left(u^{i+1}-u^{i}\right)
$$

given a step length parameter $\tau>0$. If $H$ is a $\gamma$-strongly monotone operator and $\widehat{u} \in H^{-1}(0)$, then $\left\langle H\left(u^{i+1}\right), u^{i+1}-\widehat{u}\right\rangle \geq \gamma\left\|u^{i+1}-\widehat{u}\right\|^{2}$. This suggest "testing" (2.4) by the application of $\left\langle\cdot, u^{i+1}-\widehat{u}\right\rangle$. Subsequently to this testing, the strong monotonicity and Pythagoras's identity,

$$
\left\langle u^{i+1}-u^{i}, u^{i+1}-\widehat{u}\right\rangle=\frac{1}{2}\left\|u^{i+1}-u^{i}\right\|^{2}-\frac{1}{2}\left\|u^{i}-\widehat{u}\right\|^{2}+\frac{1}{2}\left\|u^{i+1}-\widehat{u}\right\|^{2},
$$

applied to $0 \in\left\langle H\left(u^{i+1}\right)+\tau^{-1}\left(u^{i+1}-u^{i}\right), u^{i+1}-\widehat{u}\right\rangle$ yield

$$
\frac{1+2 \gamma \tau}{2}\left\|u^{i+1}-\widehat{u}\right\|^{2}+\frac{1}{2}\left\|u^{i+1}-u^{i}\right\|^{2} \leq \frac{1}{2}\left\|u^{i}-\widehat{u}\right\|^{2} .
$$

By telescoping this inequality, it is clear that $u^{N} \rightarrow \widehat{u}$ at the linear rate $O\left(1 /(1+2 \gamma \tau)^{N}\right)$. The next theorem from [34] generalises these simple arguments to the more general algorithm (PP) in the stochastic setting.

THEOREM 2.1 ([34, Corollary 3.1]). On a Hilbert space $U$ and a probability space $(\Omega, \mathcal{O})$, let $\widetilde{H}_{i+1}: \mathcal{R}(\mathcal{O} ; U \rightrightarrows U)$ and $M_{i+1}, Z_{i+1} \in \mathcal{R}(\mathcal{O} ; \mathbb{L}(U ; U))$ for $i \in \mathbb{N}$. Suppose that (PP) is solvable for $\left\{u^{i+1}\right\}_{i \in \mathbb{N}} \subset \mathcal{R}(\mathcal{O} ; U)$. If for all $i \in \mathbb{N}$ and almost all random events $\omega \in \Omega,\left(Z_{i+1} M_{i+1}\right)(\omega)$ is self-adjoint and the expected fundamental condition

$$
\begin{aligned}
& \mathbb{E}\left[\left\langle W_{i+1} \widetilde{H}_{i+1}\left(u^{i+1}\right), u^{i+1}-\widehat{u}\right\rangle_{Z_{i+1}}\right] \\
& \quad \geq \mathbb{E}\left[\frac{1}{2}\left\|u^{i+1}-\widehat{u}\right\|_{Z_{i+2} M_{i+2}-Z_{i+1} M_{i+1}}^{2}-\frac{1}{2}\left\|u^{i+1}-u^{i}\right\|_{Z_{i+1} M_{i+1}}^{2}\right]
\end{aligned}
$$

holds, then so does the expected descent inequality

$$
\mathbb{E}\left[\frac{1}{2}\left\|u^{N}-\widehat{u}\right\|_{Z_{N+1} M_{N+1}}^{2}\right] \leq \mathbb{E}\left[\frac{1}{2}\left\|u^{0}-\widehat{u}\right\|_{Z_{1} M_{1}}^{2}\right] \quad(N \geq 1) .
$$

Condition (2.5) is simply a relaxation of the strong monotonicity we assumed above. It also includes the term $\frac{1}{2}\left\|u^{i+1}-u^{i}\right\|_{Z_{i+1} M_{i+1}}$ intended to be used with forward steps. In application to (2.4), we have $M_{i+1}=I$, and we can take as the testing operator $Z_{i+1}=\phi_{i} I$ with $\phi_{i+1}=(1+2 \gamma \tau) \phi_{i}$ and $\phi_{0}=1$. Thus, $Z_{N+1} M_{N+1}$ in (2.6) forms a local metric that measures rates of convergence. If we can ensure $Z_{i+1} M_{i+1} \geq \mu_{i} I$ for some deterministic $\mu_{i} \nearrow \infty$, then (2.6) reveals that $\mathbb{E}\left[\left\|u^{N}-\widehat{u}\right\|^{2}\right]$ converges to zero at the rate $O\left(1 / \mu_{N}\right)$. We will develop lower bounds of this kind in Section 3. 


\section{ETNA}

Kent State University and

Johann Radon Institute (RICAM)

2.3. Blockwise algorithm structure. We now develop a more blockwise-refined structure of our proposed algorithm. Inserting (2.2), we can expand (PP) as the pair of implicit updates (compare $[35, \S 2.3]$ )

$$
\left\{\begin{array}{c}
x^{i+1}=\left(I+T_{i} \partial G\right)^{-1}\left(x^{i}+\Phi_{i}^{-1}\left[\Lambda_{i}^{*}-\Phi_{i} T_{i} \nabla K\left(x^{i}\right)^{*}\right]\left(y^{i+1}-y^{i}\right)\right. \\
\left.-T_{i} \nabla K\left(x^{i}\right)^{*} y^{i}\right) \\
y^{i+1}=\left(I+\Sigma_{i+1} \partial F^{*}\right)^{-1}\left(y^{i}+\Psi_{i+1}^{-1}\left[\Lambda_{i}-\Psi_{i+1} \Sigma_{i+1} \nabla K\left(x^{i}\right) \Omega^{i}\right]\left(x^{i+1}-x^{i}\right)\right. \\
\left.+\Sigma_{i+1} K\left(x^{i+1}+\Omega^{i}\left(x^{i+1}-x^{i}\right)\right)\right) .
\end{array}\right.
$$

Due to the block-separable structure of $G$ and $F^{*}$ in (2.1), we take for all $i \in \mathbb{N}$,

$$
\begin{aligned}
\text { (2.8a) } T_{i}:=\sum_{j \in S(i)} \tau_{j}^{i} P_{j}, & \Sigma_{i+1}:=\sum_{\ell \in V(i+1)} \sigma_{\ell}^{i+1} Q_{\ell}, & \Omega_{i}:=\sum_{j \in S(i)} \omega_{j}^{i} P_{j}, \\
\text { (2.8b) } \Phi_{i}:=\sum_{j=1}^{m} \phi_{j}^{i} P_{j}, & \Psi_{i+1}:=\sum_{\ell=1}^{n} \psi_{\ell}^{i+1} Q_{\ell}, & \Lambda_{i}:=\sum_{j=1}^{m} \sum_{\ell=1}^{n} \lambda_{\ell, j}^{i} Q_{\ell} \nabla K\left(x^{i}\right) P_{j},
\end{aligned}
$$

for some (random) subsets of indices $S(i) \subseteq\{1, \ldots, m\}$ and $V(i+1) \subseteq\{1, \ldots, n\}$ and (random) parameters $\tau_{j}^{i}, \phi_{j}^{i}, \sigma_{\ell}^{i+1}, \psi_{\ell}^{i+1}>0$, and $\omega_{j}^{i}, \lambda_{j, \ell}^{i} \in \mathbb{R}$. We wait until (3.14) to specify the exact probabilistic setup, which we do not need before that. Due to the blockseparable structures of $G$ and $F^{*}$, the operators $\left(I+T_{i} \partial G\right)^{-1}$ and $\left(I+\Sigma_{i+1} \partial F^{*}\right)^{-1}$ are also block-separable.

We also pick further subsets of indices $\stackrel{\circ}{S}(i) \subseteq S(i)$ and ${ }^{\circ}(i+1) \subset V(i+1)$; the rough idea is that $x_{j}^{i+1}$ for $j \in \stackrel{S}{S}(i)$ is updated within each step of the algorithm independently of $y^{i+1}$. In the linear- $K$ case of [35], also $y_{\ell}^{i+1}$ for $\ell \in \stackrel{\circ}{V}(i+1)$ would be updated independently of $x^{i+1}$, but presently we are not able to ensure that. However, we show at the end of this section that the primal blocks $x_{j}^{i+1}$ for $j \in S(i) \backslash \stackrel{S}{S}(i)$ still depend on $y_{\ell}^{i+1}$ only for $\ell \in \stackrel{\circ}{V}(i+1)$, as is the case for a linear $K$ in [35]. Moreover, we require the "nesting conditions"

$$
\begin{aligned}
\chi_{\dot{S}(i)}(j)\left(1-\chi_{V(i+1)}(\ell)\right) & =0, & \left(1-\chi_{S(i)}(j)\right) \chi_{\dot{V}(i+1)}(\ell) & =0, \\
\chi_{\dot{S}(i)}(j) \chi_{\dot{V}(i+1)}(\ell) & =0, \quad \text { and } & \chi_{S(i) \backslash \dot{S}(i)}(j) \chi_{V(i+1) \backslash \dot{V}(i+1)}(\ell) & =0,
\end{aligned}
$$

when

$$
\ell \in \mathcal{V}_{j}^{i}:=\left\{\ell \in\{1, \ldots, n\} \mid Q_{\ell} \nabla K\left(x^{i}\right) P_{j} \neq 0\right\} .
$$

These conditions force those dual blocks that are "connected" by $K$ to the "independently updated" primal blocks $\stackrel{S}{S}(i)$ to also be ("dependently") updated and vice versa. They also disallow connections between independently updated blocks and dependently updated blocks. Note that the last three equations in (2.9) are tantamount to the single equality $\chi_{V(i+1)}(\ell) \chi_{S(i) / S(i)}(j)=\chi_{\dot{V}(i+1)}(\ell)$ : they follow by multiplying the latter by $1-\chi_{S(i)}$, $\chi_{\dot{S}(i)}(j)$, and $\chi_{S(i) / S(i)}(j)$, respectively, and vice versa,

$$
\chi_{\dot{V}(i+1)}(\ell)=\chi_{\dot{V}(i+1)}(\ell) \chi_{S(i)}(j)=\chi_{\dot{V}(i+1)}(\ell) \chi_{S(i) / \stackrel{\circ}{(i)}}(j)=\chi_{V(i+1)}(\ell) \chi_{S(i) / \stackrel{\circ}{S}(i)}(j) .
$$


EXAMPLE 2.2. We can trivially satisfy (2.9) by taking either $V(i+1)=\{1, \ldots, n\}$, $\stackrel{\circ}{V}(i+1)=\emptyset$, and $\stackrel{\circ}{S}(i)=S(i)$ or $S(i)=\{1, \ldots, m\}, \stackrel{\circ}{S}(i)=\emptyset$, and $\stackrel{\circ}{V}(i+1)=V(i+1)$. We will consider these two cases in the respective Section 4 (full dual update methods) and Section 5 (full primal update methods). We may also alternate iterations between these two choices.

Following the notations for the subsets and their complements, we also write

$$
\begin{aligned}
\stackrel{\circ}{P}_{i} & :=\sum_{j \in \stackrel{\circ}{S}(i)} P_{j}, & \breve{P}_{i} & :=\sum_{j \in S(i) \backslash \grave{S}(i)} P_{j}, \\
\stackrel{\circ}{Q}_{i+1} & :=\sum_{\ell \in \stackrel{\circ}{ }(i+1)} Q_{\ell}, & \breve{Q}_{i+1} & :=\sum_{\ell \in V(i+1) \backslash \dot{V}(i+1)} Q_{\ell} .
\end{aligned}
$$

In (2.7), for the subsets $S(i)$ and $V(i+1)$ to have the intended meaning that only the corresponding blocks are updated, we need to ensure that $P_{j} x^{i+1}=P_{j} x^{i}$ for $j \notin S(i)$ and $Q_{\ell} y^{i+1}=Q_{\ell} y^{i}$ for $\ell \notin V(i+1)$. This holds if $P_{j} \Lambda_{i}^{*} Q_{\ell}=0$ whenever $j \notin S(i), \ell \in V(i+1)$ or $j \in S(i), \ell \notin V(i+1)$ or $j \notin S(i), \ell \notin V(i+1)$. Similarly, for $\stackrel{S}{S}(i)$ to have the intended meaning that $x_{j}^{i+1}$ for $j \in \dot{S}(i)$ does not depend on $y^{i+1}$, studying (2.7), we are also led to require

$$
\stackrel{\circ}{P}_{i}\left[\Lambda_{i}^{*}-\Phi_{i} T_{i} \nabla K\left(x^{i}\right)^{*}\right] Q_{\ell}=0 \text { for any } \ell \in V(i+1) .
$$

Finally, since $\breve{P}_{i} x^{i+1}$ in (2.7) may depend on $y^{i+1}$, we require $y^{i+1}$ to not depend on $\breve{P}_{i} x^{i+1}$ :

$$
\left[\Lambda_{i}-\Psi_{i+1} \Sigma_{i+1} \nabla K\left(x^{i}\right) \Omega^{i}\right] \breve{P}_{i}=0 \quad \text { and } \quad\left[I+\Omega^{i}\right] \breve{P}_{i}=0 .
$$

Combining the above conditions for $\Lambda_{i}$ and $\Omega_{i}$, we arrive at

$$
\begin{cases}P_{j} \Lambda_{i}^{*} Q_{\ell}=0 & \text { whenever either } j \notin S(i) \text { or } \\ \stackrel{\circ}{P}_{i}\left[\Lambda_{i}^{*}-\Phi_{i} T_{i} \nabla K\left(x^{i}\right)^{*}\right] Q_{\ell}=0 & \ell \notin V(i+1) \text { or both, } \\ {\left[\Lambda_{i}+\Psi_{i+1} \Sigma_{i+1} \nabla K\left(x^{i}\right)\right] \breve{P}_{i}=0,} & \text { and }\left(\Omega^{i}+I\right) \breve{P}_{i}=0 .\end{cases}
$$

Substituting (2.10) into the identity

$$
\Lambda_{i}=\sum_{\ell \in V(i+1)} Q_{\ell} \Lambda_{i} \stackrel{\circ}{P}_{i}+\sum_{\ell \notin V(i+1)} Q_{\ell} \Lambda_{i} \stackrel{\circ}{P}_{i}+\Lambda_{i} \breve{P}_{i}+\sum_{j \notin S(i)} \sum_{\ell=1}^{n} Q_{\ell} \Lambda_{i} P_{j}
$$

we are led to take

$$
\Lambda_{i}:=\sum_{\ell \in V(i+1)} Q_{\ell} \nabla K\left(x^{i}\right) T_{i}^{*} \Phi_{i}^{*} \stackrel{\circ}{P}_{i}-\Psi_{i+1} \Sigma_{i+1} \nabla K\left(x^{i}\right) \breve{P}_{i},
$$

which in terms of the components $\lambda_{\ell, j}^{i}$ reads

$$
\lambda_{\ell, j}^{i}:= \begin{cases}\tau_{j}^{i} \phi_{j}^{i} & \ell \in V(i+1), j \in \stackrel{\circ}{S}(i), \\ -\sigma_{\ell}^{i+1} \psi_{\ell}^{i+1} & \ell \in V(i+1), j \in S(i) \backslash \stackrel{\circ}{S}(i), \\ 0 & \text { otherwise }\end{cases}
$$

Using the coupling conditions (2.9) between $\stackrel{\circ}{S}(i)$ and $\stackrel{\circ}{V}^{\prime}(i+1)$ in (2.11), we deduce

$$
\Lambda_{i}=\nabla K\left(x^{i}\right) T_{i}^{*} \Phi_{i}^{*} \stackrel{\circ}{P}_{i}-\stackrel{\circ}{Q}_{i+1} \Psi_{i+1} \Sigma_{i+1} \nabla K\left(x^{i}\right) .
$$


Plugging $\Lambda_{i}$ into (2.7), we get two cases for the primal variable. If $j \in \stackrel{\circ}{S}(i)$, then we have

$$
\stackrel{\circ}{P}_{i} x^{i+1}=\left(I+\stackrel{\circ}{T}_{i} \partial G\right)^{-1}\left(\stackrel{\circ}{P}_{i} x^{i}-\stackrel{\circ}{T}_{i} \nabla K\left(x^{i}\right)^{*} y^{i}\right), \quad \text { where } \stackrel{\circ}{T}_{i}:=\stackrel{\circ}{P}_{i} T_{i} .
$$

If $j \in S(i) \backslash \stackrel{S}{S}(i)$, given that $\Omega^{i} \breve{P}_{i}=-\breve{P}_{i}$ due to the last equality of (2.10) and taking $\breve{T}_{i}:=\breve{P}_{i} T_{i}$, we have

$$
\begin{aligned}
\breve{P}_{i} x^{i+1}=\left(I+\breve{T}_{i} \partial G\right)^{-1} & \left(\breve{P}_{i} x^{i}-\breve{T}_{i} \nabla K\left(x^{i}\right)^{*} \stackrel{\circ}{Q}_{i+1} y^{i+1}\right. \\
& \left.\quad-\breve{P}_{i} \Phi_{i}^{-1} \nabla K\left(x^{i}\right)^{*} \Sigma_{i+1}^{*} \Psi_{i+1}^{*} \stackrel{\circ}{Q}_{i+1}\left(y^{i+1}-y^{i}\right)\right) .
\end{aligned}
$$

Also $x^{i+1}=\stackrel{\circ}{P}_{i} x^{i+1}+\breve{P}_{i} x^{i+1}+\left(I-\stackrel{\circ}{P}_{i}-\breve{P}_{i}\right) x^{i+1}$, therefore, for $\bar{x}^{i+1}=x^{i+1}+\Omega^{i}\left(x^{i+1}-x^{i}\right)$, we can expand

$x^{i+1}=\stackrel{\circ}{P}_{i} x^{i+1}-\Omega^{i} \breve{P}_{i} x^{i+1}+\left(I-\stackrel{\circ}{P}_{i}-\breve{P}_{i}\right) x^{i}=\stackrel{\circ}{P}_{i} x^{i+1}+\left(I-\stackrel{\circ}{P}_{i}\right) x^{i}-\Omega^{i} \breve{P}_{i}\left(x^{i+1}-x^{i}\right)$.

Consequently, the implicitly defined algorithm in (2.7) expands to the following explicit successive updates for each of the involved projections:

$$
\left\{\begin{aligned}
\stackrel{\circ}{P}_{i} x^{i+1}:= & \left(I+\stackrel{\circ}{T}_{i} \partial G\right)^{-1}\left(\stackrel{\circ}{P}_{i} x^{i}-\stackrel{\circ}{T}_{i} \nabla K\left(x^{i}\right)^{*} y^{i}\right) \\
\bar{x}^{i+1}:= & \left(I-\stackrel{\circ}{P}_{i}\right) x^{i}+\stackrel{\circ}{P}_{i} x^{i+1}+\stackrel{\circ}{P}_{i} \Omega_{i} \stackrel{\circ}{P}_{i}\left(x^{i+1}-x^{i}\right) \\
y^{i+1}:= & \left(I+\Sigma_{i+1} \partial F^{*}\right)^{-1}\left(y^{i}+\Sigma_{i+1} K\left(\bar{x}^{i+1}\right)\right. \\
& \left.\quad+\breve{Q}_{i+1} \Psi_{i+1}^{-1}\left[\nabla K\left(x^{i}\right) T_{i}^{*} \Phi_{i}^{*}-\Psi_{i+1} \Sigma_{i+1} \nabla K\left(x^{i}\right) \Omega_{i}\right] \stackrel{\circ}{P}_{i}\left(x^{i+1}-x^{i}\right)\right) \\
\breve{P}_{i} x^{i+1}:= & \left(I+\breve{T}_{i} \partial G\right)^{-1}\left(\breve{P}_{i} x^{i}-\breve{T}_{i} \nabla K\left(x^{i}\right)^{*} \stackrel{\circ}{Q}_{i+1} y^{i+1}\right. \\
\left.\quad-\breve{P}_{i} \Phi_{i}^{-1} \nabla K\left(x^{i}\right)^{*} \Sigma_{i+1}^{*} \Psi_{i+1}^{*} \stackrel{\circ}{Q}_{i+1}\left(y^{i+1}-y^{i}\right)\right) & \quad \text { for } j \notin S(i) .
\end{aligned}\right.
$$

In the following sections we will further develop and simplify this algorithm by imposing additional conditions for the step length and testing parameters through a convergence analysis.

3. General estimates. With the estimate (2.6) in mind, our main task in this section is to prove (2.5). After introducing the assumptions that we need for this work in Section 3.1, and bounding $Z_{i+1} M_{i+1}$ from below in Section 3.2, we carry out the first stage of this estimation in Section 3.3 still deterministically. Then in Section 3.4 we refine these estimates by taking the expectation. Finally, in Section 3.5 we combine the various estimates and state a self-contained result for the validity of (2.6).

3.1. Assumptions. We will need $K$ to be sufficiently smooth and to satisfy a somewhat technical "three-point" version of standard second-order growth conditions:

ASSUMPTION 3.1 (Lipschitz $\nabla K(x)$ ). For some $L \geq 0$ and a neighbourhood $\mathcal{X}_{K} \ni \widehat{x}$,

$$
\left\|\nabla K(x)-\nabla K\left(x^{\prime}\right)\right\| \leq L\left\|x-x^{\prime}\right\| \quad\left(x, x^{\prime} \in \mathcal{X}_{K}\right) .
$$

Using the equality

$$
K\left(x^{\prime}\right)=K(x)+\nabla K(x)\left(x^{\prime}-x\right)+\int_{0}^{1}\left(\nabla K\left(x+s\left(x^{\prime}-x\right)\right)-\nabla K(x)\right)\left(x^{\prime}-x\right) d s,
$$




\section{ETNA}

Kent State University and

Johann Radon Institute (RICAM)

we obtain for any $x, x^{\prime} \in \mathcal{X}_{K}$ and $y \in \operatorname{dom} F^{*}$ as a direct consequence of Assumption 3.1 that

$$
\left\langle K\left(x^{\prime}\right)-K(x)-\nabla K(x)\left(x^{\prime}-x\right), y\right\rangle \leq \frac{L}{2}\left\|x-x^{\prime}\right\|^{2}\|y\|_{P_{\mathrm{NL}}} .
$$

The norm of $y$ only needs to be evaluated within $Y_{\mathrm{NL}}$ because $x \mapsto\left(I-P_{\mathrm{NL}}\right) K(x)$ is linear, so the corresponding inner product with the integral term is zero.

ASSUMPTION 3.2 (three-point condition on $K$ ). For a neighbourhood $\mathcal{X}_{K}$ of $\widehat{x}$, some $\Gamma_{K}=\sum_{j=1}^{m} \gamma_{K, j} P_{j} \in \mathbb{L}(X ; X)$ with $\gamma_{K, j} \in \mathbb{R}, L_{3} \geq 0$, and $p \in[1,2]$, for any $A=\sum_{j=1}^{m} a_{j} P_{j} \geq 0$ and some $\theta_{A} \geq 0$, the following holds

$$
\begin{aligned}
\langle[\nabla K(x)- & \left.\nabla K(\widehat{x})]^{*} \widehat{y}, x^{\prime}-\widehat{x}\right\rangle_{A} \\
\geq\left\|x^{\prime}-\widehat{x}\right\|_{A \Gamma_{K}}^{2} & +\theta_{A}\|K(\widehat{x})-K(x)-\nabla K(x)(\widehat{x}-x)\|^{p} \\
& -\frac{L_{3}}{2}\left\|x^{\prime}-x\right\|_{A}^{2}, \quad\left(x, x^{\prime} \in \mathcal{X}_{K}\right) .
\end{aligned}
$$

This assumption is trivially satisfied for $\gamma_{K, j}=L_{3}=0$ and any $\theta_{A}>0$ whenever $x \mapsto\langle K(x), \widehat{y}\rangle$ is linear. In Appendix A we also provide the constants ensuring this assumption, e.g., whenever the latter is block-separable and strongly-convex. For a less straightforward example in the single-block case, we refer to [9]. There we verified the assumption for the reconstruction of the phase and amplitude of a complex number from noisy measurements. That example evidently applies to the present setting in the single-block case or as a separable block of $x \mapsto\langle K(x), \widehat{y}\rangle$.

We also need pointwise monotonicity of $\partial G$ and $\partial F^{*}$ at a root $\widehat{u} \in H^{-1}(0)$ :

DEFINITION 3.3. Let $U$ be a Hilbert space, and $\Gamma \in \mathbb{L}(U ; U), \Gamma \geq 0$. We say that the set-valued map $H: U \rightrightarrows U$ is $\Gamma$-strongly monotone at $\widehat{u}$ for $\widehat{w} \in H(\widehat{u})$ if there exists a neighbourhood $\mathcal{U} \ni \widehat{u}$ such that for any $u \in \mathcal{U}$ and $w \in H(u)$,

$$
\langle w-\widehat{w}, u-\widehat{u}\rangle \geq\|u-\widehat{u}\|_{\Gamma}^{2} .
$$

If $\Gamma=0$, then we say that $H$ is monotone at $\widehat{u}$ for $\widehat{w}$.

ASSUMPTION 3.4. For any $\widehat{w}=(\widehat{\nu}, \widehat{\xi}) \in H(\widehat{u})$, the set-valued map $\partial G$ is $\sum_{j=1}^{m} \gamma_{G, j} P_{j}$ strongly monotone at $\widehat{x}$ for $\widehat{\nu}-\nabla K(\widehat{x})^{*} \widehat{y}$ in the neighbourhood $\mathcal{X}_{G}$, and the set-valued map $\partial F^{*}$ is $\sum_{\ell=1}^{n} \gamma_{F^{*}, \ell} Q_{\ell^{-}}$strongly monotone at $\widehat{y}$ for $\widehat{\xi}+K(\widehat{x})$ in the neighbourhood $\mathcal{Y}_{F^{*}}$, where the constants satisfy $\gamma_{G, j}, \gamma_{F^{*}, \ell} \geq 0$, for all $j=1, \ldots, m$ and $\ell=1, \ldots, n$.

3.2. A lower bound on the local metric. To estimate $Z_{i+1} M_{i+1}$ from below, we formulate a block-adapted version of the basic step length condition $\tau \sigma\|K\|^{2}<1$ from [7]. The assumptions of the following lemma replace the more abstract constructions of [35, Definition 2.2 and Examples 2.3 and 2.4]. We recall from (2.9c) the "set of connections" $\mathcal{V}_{j}^{i}$ and also introduce the set of "simultaneous connections", filtered by $\lambda_{k, j}^{i}$, as

$$
\overline{\mathcal{V}}_{j}^{i}(\ell):=\left\{k \in\{1, \ldots, n\} \mid Q_{\ell} \nabla K\left(x^{i}\right) P_{j} \nabla K\left(x^{i}\right)^{*} Q_{k} \neq 0, \lambda_{k, j}^{i} \neq 0\right\} .
$$

Lemma 3.5. Let $i \in \mathbb{N}$ and $0 \leq \delta \leq \kappa<1$. For some weights $w_{j, \ell, k}^{i}=1 / w_{j, k, \ell}^{i}>0$ $(\ell, k=1, \ldots, n, j=1, \ldots, m)$, define

$$
w_{j, \ell}^{i}:=\chi_{\mathcal{V}_{j}^{i}}(\ell) \sum_{k \in \overline{\mathcal{V}}_{j}^{i}(\ell)} w_{j, \ell, k}^{i},
$$


and suppose that

$$
(1-\kappa) \psi_{\ell}^{i+1} \geq\left\|\sum_{j=1}^{m}\left|\lambda_{\ell, j}^{i}\right| \sqrt{w_{j, \ell}^{i} / \phi_{j}^{i}} Q_{\ell} \nabla K\left(x^{i}\right) P_{j}\right\|^{2} \quad(\ell=1, \ldots, n) .
$$

Then

$$
Z_{i+1} M_{i+1} \geq\left[\begin{array}{cc}
\delta \Phi_{i} & 0 \\
0 & \frac{\kappa-\delta}{1-\delta} \Psi_{i+1}
\end{array}\right]
$$

Proof. Setting $\zeta_{\ell, j}:=\left(\phi_{j}^{i}\right)^{-1}\left(\lambda_{\ell, j}^{i}\right)^{2} /(1-\kappa)$, we use (3.5) and the orthogonality of the projections $\left\{P_{j}\right\}_{j=1}^{m}$ to obtain for any $y \in Y$ that

$$
\begin{aligned}
\sum_{\ell=1}^{n} \psi_{\ell}^{i+1}\left\|Q_{\ell} y\right\|^{2} & \geq \sum_{\ell=1}^{n}\left\|\sum_{j=1}^{m} \sqrt{\zeta_{\ell, j} w_{j, \ell}^{i}} Q_{\ell} \nabla K\left(x^{i}\right) P_{j}\right\|^{2}\left\|Q_{\ell} y\right\|^{2} \\
& \geq \sum_{\ell=1}^{n}\left\|\sum_{j=1}^{m} \sqrt{\zeta_{\ell, j} w_{j, \ell}^{i}} P_{j} \nabla K\left(x^{i}\right)^{*} Q_{\ell} y\right\|^{2} \\
& =\sum_{\ell=1}^{n} \sum_{j=1}^{m} \zeta_{\ell, j} w_{j, \ell}^{i}\left\|P_{j} \nabla K\left(x^{i}\right)^{*} Q_{\ell} y\right\|^{2} \\
& \geq \sum_{j=1}^{m} \sum_{\ell \in \mathcal{V}_{j}^{i}}\left(\sum_{k \in \overline{\mathcal{V}}_{j}^{i}(\ell)} w_{j, \ell, k}^{i}\right) \zeta_{\ell, j}\left\|P_{j} \nabla K\left(x^{i}\right)^{*} Q_{\ell} y\right\|^{2} .
\end{aligned}
$$

Since $w_{j, k, \ell}^{i}=1 / w_{j, \ell, k}^{i}$, we continue to estimate by Young's inequality

$$
\sum_{\ell=1}^{n} \psi_{\ell}^{i+1}\left\|Q_{\ell} y\right\|^{2} \geq \sum_{j=1}^{m} \sum_{k, \ell=1}^{n} \zeta_{\ell, j}^{1 / 2} \zeta_{k, j}^{1 / 2}\left\langle P_{j} \nabla K\left(x^{i}\right)^{*} Q_{\ell} y, \nabla K\left(x^{i}\right)^{*} Q_{k} y\right\rangle .
$$

Here we also used (3.3) to convert the second sum to run over all $k, \ell=1, \ldots, n$. As $y \in Y$ was arbitrary, inserting $\zeta_{k, j}$ and the structure (2.8) of $\Psi_{i+1}, \Phi_{i}$, and $\Lambda_{i}$, we deduce that $(1-\kappa) \Psi_{i+1} \geq \Lambda_{i} \Phi_{i}^{-1} \Lambda_{i}^{*}$.

On the other hand, applying Young's inequality with the factor $(1-\delta)$, we deduce that

$$
Z_{i+1} M_{i+1}=\left[\begin{array}{cc}
\Phi_{i} & -\Lambda_{i}^{*} \\
-\Lambda_{i} & \Psi_{i+1}
\end{array}\right] \geq\left[\begin{array}{cc}
\delta \Phi_{i} & 0 \\
0 & \Psi_{i+1}-\frac{1}{1-\delta} \Lambda_{i} \Phi_{i}^{-1} \Lambda_{i}^{*}
\end{array}\right]
$$

Thus, (3.6) holds.

The next example demonstrates a simple choice of the weights $w_{j, k, \ell}$ that is likely to work if all the dual blocks $\ell$ have similar roles in the problem. In Section 6 we will also consider other options when some dual blocks have different roles.

EXAMPLE 3.6 (Equal weighting). Suppose $\mathcal{V}_{j}^{i} \subset \mathcal{V}_{j}$ and $\overline{\mathcal{V}}_{j}^{i}(\ell) \subset \overline{\mathcal{V}}_{j}(\ell)$, where $\mathcal{V}_{j}$ and $\overline{\mathcal{V}}_{j}(\ell)$ do not depend on the iteration. If we take $w_{j, \ell, k}^{i} \equiv 1$, then $w_{j, \ell}=\chi_{\mathcal{V}_{j}}(\ell) \# \overline{\mathcal{V}}_{j}(\ell)$ counts the dual blocks "simultaneously connected" with $\ell$ via the primal block $j$ as defined by (3.3).

To provide further intuition of the result, let $w_{j, \ell}$ be as in Theorem 3.6. With only one primal block $(j, m=1)$ and assuming full connectedness $\left(w_{1, \ell}=n\right.$ for all $\left.\ell=1, \ldots, n\right)$, Theorem 3.5 requires $\psi_{\ell} \geq \zeta_{1, \ell} n\left\|Q_{\ell} \nabla K\left(x^{i}\right)\right\|^{2}$. Let $a:=\frac{1}{n} \sum_{\ell=1}^{n}\left\|Q_{\ell} \nabla K\left(x^{i}\right)\right\|^{2}=$ 
$\frac{1}{n}\left\|\nabla K\left(x^{i}\right)\right\|^{2}$. After plugging in $\lambda_{\ell, j}^{i}$ from (2.12) into (3.5), the lemma then says that the step length parameters can be proportionally larger compared to the single dual block case $(n=1)$ when $\left\|Q_{\ell} \nabla K\left(x^{i}\right)\right\|^{2}<a$ and have to be proportionally smaller when $\left\|Q_{\ell} \nabla K\left(x^{i}\right)\right\|^{2}>a$. In Section 4 and Section 5, we further transform (3.5) to obtain explicit step-length conditions. But now, for the remainder of Section 3, we assume that (3.6) holds, and we derive sufficient conditions for being able to apply Theorem 2.1.

3.3. Initial non-stochastic estimates. The next lemma starts the verification of (2.5).

LEMMA 3.7. Suppose that Assumptions 3.1 and 3.4 hold together with (3.6) for some $L \geq 0, \gamma_{G, j}, \gamma_{F^{*}, \ell} \geq 0(j=1, \ldots, m, \ell=1, \ldots, n)$, and $0 \leq \delta \leq \kappa<1$. Then, with $\widetilde{H}_{i+1}$ given by (2.3) and $M_{i+1}$ given by (2.2), we have

$$
\begin{aligned}
\frac{1}{2}\left\|u^{i+1}-u^{i}\right\|_{Z_{i+1} M_{i+1}}^{2} & +\frac{1}{2}\left\|u^{i+1}-\widehat{u}\right\|_{Z_{i+1} M_{i+1}-Z_{i+2} M_{i+2}}^{2} \\
& +\left\langle\widetilde{H}_{i+1}\left(u^{i+1}\right), u^{i+1}-\widehat{u}\right\rangle_{W_{i+1} Z_{i+1}} \\
\geq & \frac{1}{2}\left\|x^{i+1}-x^{i}\right\|_{R_{x}}^{2}+\frac{1}{2} \frac{\kappa-\delta}{1-\delta}\left\|y^{i+1}-y^{i}\right\|_{\Psi_{i+1}}^{2} \\
& +\frac{1}{2}\left\|u^{i+1}-\widehat{u}\right\|_{R^{\prime}}^{2}+D_{i}^{K}+D_{i}^{\Lambda},
\end{aligned}
$$

where for an arbitrary $\Gamma_{K}:=\sum_{j=1}^{m} \gamma_{K, j} P_{j} \in \mathbb{L}(X ; X)$ for $\gamma_{K, j} \in \mathbb{R}$ we set

$$
\begin{aligned}
& R_{x}:=\delta \Phi_{i}-L\left\|\Omega^{i}+I\right\|^{2}\left\|\Psi_{i+1}^{*} \Sigma_{i+1}^{*}\left(y^{i+1}-\widehat{y}\right)\right\|_{P_{\mathrm{NL}}} I, \\
& R^{\prime}:= \\
& {\left[\begin{array}{cc}
\Phi_{i}-\Phi_{i+1}+2 \sum_{j \in S(i)} \phi_{j}^{i} \tau_{j}^{i}\left(\gamma_{G, j}+\gamma_{K, j}\right) P_{j} & 0 \\
0 & \Psi_{i+1}-\Psi_{i+2}+2 \sum_{\ell \in V(i+1)} \psi_{\ell}^{i+1} \sigma_{\ell}^{i+1} \gamma_{F^{*}, \ell} Q_{\ell}
\end{array}\right],} \\
& \text { (3.8c) } D_{i}^{\Lambda}:=\left\langle\left[\Lambda_{i+1}-\Lambda_{i}\right]\left(x^{i+1}-\widehat{x}\right), y^{i+1}-\widehat{y}\right\rangle \\
& +\left\langle\nabla K\left(x^{i}\right)^{*}\left(y^{i+1}-\widehat{y}\right), x^{i+1}-\widehat{x}\right\rangle_{\Phi_{i} T_{i}-\Sigma_{i+1}^{*} \Psi_{i+1}^{*}}, \\
& \text { (3.8d) } D_{i}^{K}:=\left\langle\left[\nabla K\left(x^{i}\right)-\nabla K(\widehat{x})\right]^{*} \widehat{y}, x^{i+1}-\widehat{x}\right\rangle_{\Phi_{i} T_{i}}-\left\|x^{i+1}-\widehat{x}\right\|_{\Phi_{i} T_{i} \Gamma_{K}}^{2} \\
& +\left\langle K(\widehat{x})-K\left(x^{i}\right)-\nabla K\left(x^{i}\right)\left(\widehat{x}-x^{i}\right), y^{i+1}-\widehat{y}\right\rangle_{\Psi_{i+1} \Sigma_{i+1}} .
\end{aligned}
$$

Proof. We bound from below all the terms on the left-hand side of (3.7). For the first term, we have from (3.6) that

$$
Z_{i+1} M_{i+1} \geq\left[\begin{array}{cc}
\delta \Phi_{i} & 0 \\
0 & \frac{\kappa-\delta}{1-\delta} \Psi_{i+1}
\end{array}\right]
$$

For the second term we use the expansion

$$
Z_{i+1} M_{i+1}-Z_{i+2} M_{i+2}=\left[\begin{array}{cc}
\Phi_{i}-\Phi_{i+1} & \Lambda_{i+1}^{*}-\Lambda_{i}^{*} \\
\Lambda_{i+1}-\Lambda_{i} & \Psi_{i+1}-\Psi_{i+2}
\end{array}\right] .
$$

We need to work more to estimate the third term on the left-hand side of (3.7). Since $0 \in H(\widehat{u})$, we have $\partial G(\widehat{x}) \ni z_{G}:=-\nabla K(\widehat{x})^{*} \widehat{y}$, and $\partial F^{*}(\widehat{y}) \ni z_{F^{*}}:=K(\widehat{x})$. We can therefore recall the definition of $H(u)$ from (2.1) and rewrite

$$
\begin{aligned}
\langle H(u), u-\widehat{u}\rangle_{W_{i+1} Z_{i+1}} & \\
=\langle & \left.\partial G(x)-z_{G}, x-\widehat{x}\right\rangle_{\Phi_{i} T_{i}}+\left\langle\partial F^{*}(y)-z_{F^{*}}, y-\widehat{y}\right\rangle_{\Psi_{i+1} \Sigma_{i+1}} \\
\quad & \quad\left\langle\left\langle\nabla K(x)^{*} y-\nabla K(\widehat{x})^{*} \widehat{y}, x-\widehat{x}\right\rangle_{\Phi_{i} T_{i}}+\langle K(\widehat{x})-K(x), y-\widehat{y}\rangle_{\Psi_{i+1} \Sigma_{i+1}} .\right.
\end{aligned}
$$


Recalling the definition of $\widetilde{H}_{i+1}\left(u^{i+1}\right)$ in (2.3), we therefore expand the third term of (3.7) as

$$
\begin{aligned}
\left\langle\widetilde{H}_{i+1}\left(u^{i+1}\right), u^{i+1}-\widehat{u}\right\rangle_{W_{i+1} Z_{i+1}} & \\
=\langle & \left.\partial G\left(x^{i+1}\right)-z_{G}, x^{i+1}-\widehat{x}\right\rangle_{\Phi_{i} T_{i}}+\left\langle\partial F^{*}\left(y^{i+1}\right)-z_{F^{*}}, y^{i+1}-\widehat{y}\right\rangle_{\Psi_{i+1} \Sigma_{i+1}} \\
& +\left\langle\nabla K\left(x^{i+1}\right)^{*} y^{i+1}-\nabla K(\widehat{x})^{*} \widehat{y}, x^{i+1}-\widehat{x}\right\rangle_{\Phi_{i} T_{i}} \\
& +\left\langle K(\widehat{x})-K\left(x^{i+1}\right), y^{i+1}-\widehat{y}\right\rangle_{\Psi_{i+1} \Sigma_{i+1}} \\
& +\left\langle\left[\nabla K\left(x^{i}\right)-\nabla K\left(x^{i+1}\right)\right]^{*} y^{i+1}, x^{i+1}-\widehat{x}\right\rangle_{\Phi_{i} T_{i}} \\
& +\left\langle K\left(x^{i+1}\right)-K\left(x^{i+1}+\Omega^{i}\left(x^{i+1}-x^{i}\right)\right)\right. \\
& \left.+\nabla K\left(x^{i}\right) \Omega^{i}\left(x^{i+1}-x^{i}\right), y^{i+1}-\widehat{y}\right\rangle_{\Psi_{i+1} \Sigma_{i+1}} .
\end{aligned}
$$

Due to Assumption 3.4 and (3.1), we have

$$
\begin{gathered}
D_{i}^{\Gamma}:=\left\langle\partial G\left(x^{i+1}\right)-z_{G}, x^{i+1}-\widehat{x}\right\rangle_{\Phi_{i} T_{i}}+\left\|x^{i+1}-\widehat{x}\right\|_{\Phi_{i} T_{i} \Gamma_{K}}^{2} \\
+\left\langle\partial F^{*}\left(y^{i+1}\right)-z_{F^{*}}, y^{i+1}-\widehat{y}\right\rangle_{\Psi_{i+1} \Sigma_{i+1}} \\
\geq \sum_{j \in S(i)} \phi_{j}^{i} \tau_{j}^{i}\left\|x^{i+1}-\widehat{x}\right\|_{P_{j} \Gamma_{G} P_{j}}^{2}+\left\|x^{i+1}-\widehat{x}\right\|_{\Phi_{i} T_{i} \Gamma_{K}}^{2} \\
\quad+\sum_{\ell \in V(i+1)} \psi_{\ell}^{i+1} \sigma_{\ell}^{i+1}\left\|y^{i+1}-\widehat{y}\right\|_{Q_{\ell} \Gamma_{F^{*}} Q_{\ell}}^{2},
\end{gathered}
$$

and

$$
\begin{aligned}
D_{i}^{\Omega}:=\left\langle K\left(x^{i}\right)-K\left(x^{i+1}+\Omega^{i}\left(x^{i+1}-x^{i}\right)\right)\right. \\
\left.+\nabla K\left(x^{i}\right)\left(\Omega^{i}+I\right)\left(x^{i+1}-x^{i}\right), y^{i+1}-\widehat{y}\right\rangle_{\Psi_{i+1} \Sigma_{i+1}} \\
\geq-\frac{L}{2}\left\|\Omega^{i}+I\right\|^{2}\left\|\Psi_{i+1}^{*} \Sigma_{i+1}^{*}\left(y^{i+1}-\widehat{y}\right)\right\|_{P_{\mathrm{NL}}}\left\|x^{i+1}-x^{i}\right\|^{2} .
\end{aligned}
$$

Hence, recalling $D_{i}^{K}$ from (3.8d), we deduce

$$
\begin{aligned}
\left\langle\widetilde{H}_{i+1}\left(u^{i+1}\right), u^{i+1}-\widehat{u}\right\rangle_{W_{i+1} Z_{i+1}} & \\
=\langle[ & \left.\left.\nabla K\left(x^{i}\right)-\nabla K(\widehat{x})\right]^{*} \widehat{y}, x^{i+1}-\widehat{x}\right\rangle_{\Phi_{i} T_{i}}-\left\|x^{i+1}-\widehat{x}\right\|_{\Phi_{i} T_{i} \Gamma_{K}}^{2} \\
& +\left\langle K(\widehat{x})-K\left(x^{i}\right)-\nabla K\left(x^{i}\right)\left(\widehat{x}-x^{i}\right), y^{i+1}-\widehat{y}\right\rangle_{\Psi_{i+1} \Sigma_{i+1}} \\
& +\left\langle\partial G\left(x^{i+1}\right)-z_{G}, x^{i+1}-\widehat{x}\right\rangle_{\Phi_{i} T_{i}}+\left\|x^{i+1}-\widehat{x}\right\|_{\Phi_{i} T_{i} \Gamma_{K}}^{2} \\
& +\left\langle\partial F^{*}\left(y^{i+1}\right)-z_{F^{*}}, y^{i+1}-\widehat{y}\right\rangle_{\Psi_{i+1} \Sigma_{i+1}} \\
& +\left\langle K\left(x^{i}\right)-K\left(x^{i+1}+\Omega^{i}\left(x^{i+1}-x^{i}\right)\right)\right. \\
& \left.+\nabla K\left(x^{i}\right)\left(\Omega^{i}+I\right)\left(x^{i+1}-x^{i}\right), y^{i+1}-\widehat{y}\right\rangle_{\Psi_{i+1} \Sigma_{i+1}} \\
& +\left\langle\nabla K\left(x^{i}\right)^{*}\left(y^{i+1}-\widehat{y}\right), x^{i+1}-\widehat{x}\right\rangle_{\Phi_{i} T_{i}} \\
& -\left\langle\nabla K\left(x^{i}\right)\left(x^{i+1}-\widehat{x}\right), y^{i+1}-\widehat{y}\right\rangle_{\Psi_{i+1} \Sigma_{i+1}} \\
= & D_{i}^{K}+D_{i}^{\Gamma}+D_{i}^{\Omega}+\left\langle\nabla K\left(x^{i}\right)^{*}\left(y^{i+1}-\widehat{y}\right), x^{i+1}-\widehat{x}\right\rangle_{\Phi_{i} T_{i}-\Sigma_{i+1}^{*} \Psi_{i+1}^{*}} .
\end{aligned}
$$


Inserting the lower bounds from (3.9), (3.11), and (3.12) into (3.13) and using (3.8d) and (3.10), we obtain

$$
\begin{aligned}
\frac{1}{2}\left\|u^{i+1}-u^{i}\right\|_{Z_{i+1} M_{i+1}}^{2}+ & \frac{1}{2}\left\|u^{i+1}-\widehat{u}\right\|_{Z_{i+1} M_{i+1}-Z_{i+2} M_{i+2}}^{2} \\
+ & \left\langle\widetilde{H}_{i+1}\left(u^{i+1}\right), u^{i+1}-\widehat{u}\right\rangle_{W_{i+1} Z_{i+1}} \\
\geq \frac{1}{2}\left\|x^{i+1}-x^{i}\right\|_{\delta \Phi_{i}}^{2} & +\frac{1}{2} \frac{\kappa-\delta}{1-\delta}\left\|y^{i+1}-y^{i}\right\|_{\Psi_{i+1}}^{2}+\frac{1}{2}\left\|u^{i+1}-\widehat{u}\right\|_{R^{\prime}}^{2}+D_{i}^{\Lambda}+D_{i}^{K} \\
& -\frac{L}{2}\left\|\Omega^{i}+I\right\|^{2}\left\|\Psi_{i+1}^{*} \Sigma_{i+1}^{*}\left(y^{i+1}-\widehat{y}\right)\right\|_{P_{\mathrm{NL}}}\left\|x^{i+1}-x^{i}\right\|^{2}
\end{aligned}
$$

for $D_{i}^{\Lambda}$ as in (3.8c). Finally, using the definitions of $R_{x}$ in (3.8), we observe

$$
\begin{aligned}
& \frac{1}{2}\left\|x^{i+1}-x^{i}\right\|_{\delta \Phi_{i}}^{2}-L\left\|\Omega^{i}+I\right\|^{2}\left\|\Psi_{i+1}^{*} \Sigma_{i+1}^{*}\left(y^{i+1}-\widehat{y}\right)\right\|_{P_{\mathrm{NL}}}\left\|x^{i+1}-x^{i}\right\|^{2} \\
& =\left\|x^{i+1}-x^{i}\right\|_{R_{x}}^{2} .
\end{aligned}
$$

This yields the claim.

3.4. Expectation estimates. To further estimate $D_{i}^{K}$ and $D_{i}^{\Lambda}$, we have to take the expectation with respect to $\mathcal{O}_{i-1}$. We will use a split definition of the step lengths, writing

$$
\tau_{j}^{i}=\left\{\begin{array}{ll}
\stackrel{\circ}{\tau}_{j}^{i}, & j \in \stackrel{\circ}{S}(i), \\
\breve{\tau}_{j}^{i}, & j \in S(i) \backslash \stackrel{\circ}{S}(i),
\end{array} \quad \text { and } \quad \sigma_{\ell}^{i+1}= \begin{cases}\stackrel{\circ}{\sigma}_{\ell}^{i+1}, & \ell \in \stackrel{\circ}{ }(i+1), \\
\breve{\sigma}_{\ell}^{i+1}, & \ell \in V(i+1) \backslash \stackrel{\circ}{V}(i+1),\end{cases}\right.
$$

where we make for all $i \in \mathbb{N}$ the conditionality assumptions

$$
\begin{array}{rlrl}
\phi_{j}^{i}, \psi_{\ell}^{i+1} & \in \mathcal{R}\left(\mathcal{O}_{i-1} ;(0, \infty)\right), & \dot{\circ}_{j}^{i}, \breve{\tau}_{j}^{i}, \stackrel{\circ}{\sigma}_{\ell}^{i+1}, \breve{\sigma}_{\ell}^{i+1} & \in \mathcal{R}\left(\mathcal{O}_{i-1} ;(0, \infty)\right), \\
S(i), \stackrel{\stackrel{S}{S}(i)}{ } & \in \mathcal{R}\left(\mathcal{O}_{i} ; \mathcal{P}\{1, \ldots, m\}\right), \quad V(i+1), \stackrel{\circ}{V}(i+1) & \in \mathcal{R}\left(\mathcal{O}_{i} ; \mathcal{P}\{1, \ldots, n\}\right) .
\end{array}
$$

Thus, $\stackrel{\circ}{j}_{j}^{i}$ always refers to what $\tau_{j}^{i}$ would be if $j \in \stackrel{\circ}{S}(i)$, and similarly for the other variables. Moreover, these step lengths are already known at iteration $i-1$, prior to their use. The only part that is not known about $T_{i}$ and $\Sigma_{i+1}$ before commencing iteration $i$ are the subsets of blocks to be updated. Observe that (3.14) and (2.13) imply

$$
x^{i+1} \in \mathcal{R}\left(\mathcal{O}_{i} ; X\right) \quad \text { and } \quad y^{i+1} \in \mathcal{R}\left(\mathcal{O}_{i} ; Y\right) \quad(i \in \mathbb{N}) .
$$

Also, for brevity, we write

$$
\begin{aligned}
\pi_{j}^{i} & :=\mathbb{P}\left[j \in S(i) \mid \mathcal{O}_{i-1}\right], & \stackrel{\circ}{\pi}_{j}^{i} & :=\mathbb{P}\left[j \in \stackrel{\circ}{S}(i) \mid \mathcal{O}_{i-1}\right], \\
\nu_{\ell}^{i+1} & :=\mathbb{P}\left[\ell \in V(i+1) \mid \mathcal{O}_{i-1}\right], & \stackrel{\circ}{\ell}_{\ell}^{i+1} & :=\mathbb{P}\left[\ell \in \stackrel{\circ}{V}(i+1) \mid \mathcal{O}_{i-1}\right] .
\end{aligned}
$$

Lemma 3.8. Suppose that Assumption 3.2 and (3.14) hold for some $L_{3} \geq 0, p \in[1,2]$, and $\theta_{A} \geq 0$. For some $\rho_{\ell}>0$, assume that

$$
1=\mathbb{P}\left[\left\|y_{\ell}^{i+1}-\widehat{y}_{\ell}\right\|_{P_{\mathrm{NL}}} \leq \rho_{\ell} \mid \mathcal{O}_{i-1}\right] \quad(\ell=1, \ldots, m) .
$$

Then $D_{i}^{K}$ defined in (3.8c) satisfies for any $\zeta_{\ell}>0$ with

$$
\sum_{\ell=1}^{n} \nu_{\ell}^{i+1} \psi_{\ell}^{i+1} \sigma_{\ell}^{i+1} \zeta_{\ell}^{1-p} \rho_{\ell}^{2-p} \leq p^{p} \mathbb{E}_{i-1}\left[\theta_{\Phi_{i} T_{i}}\right]
$$


the lower bound

$$
\begin{aligned}
\mathbb{E}_{i-1}\left[D_{i}^{K}\right] \geq & -\frac{L_{3}}{2} \mathbb{E}_{i-1}\left[\left\|x^{i+1}-x^{i}\right\|_{\Phi_{i} T_{i}}^{2}\right] \\
& -\sum_{\ell=1}^{n} \mathbb{E}_{i-1}\left[\psi_{\ell}^{i+1} \sigma_{\ell}^{i+1}(p-1) \zeta_{\ell}\left\|y_{\ell}^{i+1}-\widehat{y}_{\ell}\right\|_{P_{\mathrm{NL}}}^{2}\right] .
\end{aligned}
$$

Proof. Setting $A=\Phi_{i} T_{i}$ in Assumption 3.2, we obtain

$$
\begin{gathered}
\left\langle\left[\nabla K\left(x^{i}\right)-\nabla K(\widehat{x})\right]^{*} \widehat{y}, x^{i+1}-\widehat{x}\right\rangle_{\Phi_{i} T_{i}} \\
\geq\left\|x^{i+1}-\widehat{x}\right\|_{\Phi_{i} T_{i} \Gamma_{K}}^{2}+\theta_{\Phi_{i} T_{i}}\left\|K(\widehat{x})-K\left(x^{i}\right)-\nabla K\left(x^{i}\right)\left(\widehat{x}-x^{i}\right)\right\|^{p} \\
-\frac{L_{3}}{2}\left\|x^{i+1}-x^{i}\right\|_{\Phi_{i} T_{i}}^{2} .
\end{gathered}
$$

Therefore, recalling the definition of $D_{k}^{K}$ in (3.8d) and using (3.15),

(3.18) $\mathbb{E}_{i-1}\left[D_{i}^{K}\right] \geq \mathbb{E}_{i-1}\left[\theta_{\Phi_{i} T_{i}}\right]\left\|K(\widehat{x})-K\left(x^{i}\right)-\nabla K\left(x^{i}\right)\left(\widehat{x}-x^{i}\right)\right\|^{p}$

$$
\begin{aligned}
& -\frac{L_{3}}{2} \mathbb{E}_{i-1}\left[\left\|x^{i+1}-x^{i}\right\|_{\Phi_{i} T_{i}}^{2}\right] \\
& +\left\langle K(\widehat{x})-K\left(x^{i}\right)-\nabla K\left(x^{i}\right)\left(\widehat{x}-x^{i}\right), \mathbb{E}_{i-1}\left[\sum_{i+1}^{*} \Psi_{i+1}^{*}\left(y^{i+1}-\widehat{y}\right)\right]\right\rangle .
\end{aligned}
$$

By Young's inequality and (3.16) as in [9, (3.16) and (3.17)], for any $\zeta_{\ell}>0$,

$$
\begin{aligned}
& \left\langle K(\widehat{x})-K\left(x^{i}\right)-\nabla K\left(x^{i}\right)\left(\widehat{x}-x^{i}\right), \Sigma_{i+1}^{*} \Psi_{i+1}^{*}\left(y^{i+1}-\widehat{y}\right)\right\rangle \\
& \geq-\sum_{\ell \in V(i+1)} \psi_{\ell}^{i+1} \sigma_{\ell}^{i+1}\left\|y_{\ell}^{i+1}-\widehat{y}_{\ell}\right\|_{P_{\mathrm{NL}}} \cdot\left\|K(\widehat{x})-K\left(x^{i}\right)-\nabla K\left(x^{i}\right)\left(\widehat{x}-x^{i}\right)\right\| \\
& \geq-\sum_{\ell \in V(i+1)} \psi_{\ell}^{i+1} \sigma_{\ell}^{i+1}(p-1) \zeta_{\ell}\left\|y_{\ell}^{i+1}-\widehat{y}_{\ell}\right\|_{P_{\mathrm{NL}}}^{2} \\
& \quad-\sum_{i=1}^{n} \frac{\chi_{V(i+1)}(\ell) \psi_{\ell}^{i+1} \sigma_{\ell}^{i+1}\left\|y_{\ell}^{i+1}-\widehat{y}_{\ell}\right\|_{P_{\mathrm{NL}}}^{2-p}}{p^{p} \zeta_{\ell}^{p-1}} \cdot\left\|K(\widehat{x})-K\left(x^{i}\right)-\nabla K\left(x^{i}\right)\left(\widehat{x}-x^{i}\right)\right\|^{p} .
\end{aligned}
$$

Taking the expectation $\mathbb{E}_{i-1}$, applying the assumed bound

$$
\sum_{\ell=1}^{n} \nu_{\ell}^{i+1} \psi_{\ell}^{i+1} \sigma_{\ell}^{i+1} \zeta_{\ell}^{1-p} \rho_{\ell}^{2-p} \leq p^{p} \mathbb{E}_{i-1}\left[\theta_{\Phi_{i} T_{i}}\right],
$$

and inserting the result in (3.18), we obtain the claim (3.17).

Lemma 3.9. Suppose that Assumption 3.1 and (3.14) are satisfied for some $L \geq 0$, and the nesting conditions (2.9) hold for any $j$ and $\ell$ in both iterations $i$ and $i+1$. For some $\eta^{i+1}>0$ assume that

$$
\begin{aligned}
& \stackrel{\circ}{j}_{j}^{i+1} \phi_{j}^{i+1} \stackrel{\circ}{j}_{j}^{i+1}=\eta^{i+1}-\chi_{S(i) \backslash \stackrel{\leftrightarrow}{S}(i)}(j) \phi_{j}^{i} \breve{\tau}_{j}^{i}, \\
& \stackrel{\circ}{\nu}_{\ell}^{i+2} \psi_{\ell}^{i+2} \stackrel{\circ}{\ell}_{\ell}^{i+2}=\eta^{i+1}-\chi_{V(i+1) \backslash \dot{V}(i+1)}(\ell) \psi_{\ell}^{i+1} \breve{\sigma}_{\ell}^{i+1} .
\end{aligned}
$$

Then $D_{i}^{\Lambda}$ defined in (3.8c) satisfies for any given $\alpha_{x}, \alpha_{y}>0$ the lower bound

$$
\begin{aligned}
\mathbb{E}_{i}\left[D_{i}^{\Lambda}\right]+ & \frac{d^{i+1}}{2}\left\|x^{i+1}-x^{i}\right\|^{2} \\
\geq & -\alpha_{x} \sum_{j=1}^{m} \chi_{S(i) \backslash \grave{S}(i)}(j) \phi_{j}^{i} \breve{\tau}_{j}^{i}\left\|x_{j}^{i+1}-\widehat{x}_{j}\right\|^{2} \\
& \quad-\alpha_{y} \sum_{\ell=1}^{n} \chi_{V(i+1) \backslash \grave{V}(i+1)}(\ell) \psi_{\ell}^{i+1} \breve{\sigma}_{\ell}^{i+1}\left\|y_{\ell}^{i+1}-\widehat{y}_{\ell}\right\|_{P_{\mathrm{NL}}}^{2},
\end{aligned}
$$


where

$$
\begin{aligned}
d^{i+1}:=\frac{L^{2}}{2 \alpha_{x}}\left(\sum_{j \in S(i) \backslash \grave{S}(i)} \phi_{j}^{i} \breve{\tau}_{j}^{i}\right)\left\|y^{i+1}-\widehat{y}\right\|_{P_{\mathrm{NL}}}^{2} \\
\quad+\frac{L^{2}}{2 \alpha_{y}}\left(\sum_{\ell \in V(i+1) \backslash \grave{V}(i+1)} \psi_{\ell}^{i+1} \breve{\sigma}_{\ell}^{i+1}\right)\left\|x^{i+1}-\widehat{x}\right\|^{2} .
\end{aligned}
$$

Moreover, if

$$
\mathbb{P}\left[\left\|x^{i+1}-\widehat{x}\right\| \leq \rho_{x},\left\|Q_{\ell}\left(y^{i+1}-\widehat{y}\right)\right\|_{P_{\mathrm{NL}}} \leq \rho_{\ell}, \quad(\ell=1, \ldots, n) \mid \mathcal{O}_{i-1}\right]=1,
$$

then

$$
\mathbb{E}_{i-1}\left[d^{i+1}\left\|x^{i+1}-x^{i}\right\|^{2}\right] \leq \mathbb{E}_{i-1}\left[c_{*}^{i}\left\|x^{i+1}-x^{i}\right\|^{2}\right]
$$

for

$$
\begin{aligned}
c_{*}^{i}:=\frac{L^{2}}{2 \alpha_{x} \alpha_{y}}( & \alpha_{y} \sum_{\ell=1}^{n} \rho_{\ell}^{2} \#(S(i) \backslash \stackrel{\circ}{S}(i)) \max _{j=1, \ldots, m} \phi_{j}^{i} \breve{\tau}_{j}^{i} \\
& \left.+\alpha_{x} \rho_{x}^{2} \#(V(i+1) \backslash \stackrel{\circ}{V}(i+1)) \max _{\ell=1, \ldots, n} \psi_{\ell}^{i+1} \breve{\sigma}_{\ell}^{i+1}\right) .
\end{aligned}
$$

Proof. We recall from (3.8c) that

$$
\begin{aligned}
D_{i}^{\Lambda}:= & \left\langle\nabla K\left(x^{i}\right)^{*}\left(y^{i+1}-\widehat{y}\right), x^{i+1}-\widehat{x}\right\rangle_{\Phi_{i} T_{i}-\Sigma_{i+1}^{*} \Psi_{i+1}^{*}} \\
& +\left\langle\left[\sum_{\ell \in V(i+2)} Q_{\ell} \nabla K\left(x^{i+1}\right) T_{i+1}^{*} \Phi_{i+1}^{*} \stackrel{\circ}{P}_{i+1}\right.\right. \\
& \left.\left.\quad-\Psi_{i+2} \Sigma_{i+2} \nabla K\left(x^{i+1}\right) \breve{P}_{i+1}\right]\left(x^{i+1}-\widehat{x}\right), y^{i+1}-\widehat{y}\right\rangle \\
& -\left\langle\left[\sum_{\ell \in V(i+1)} Q_{\ell} \nabla K\left(x^{i}\right) T_{i}^{*} \Phi_{i}^{*} \stackrel{\circ}{P}_{i}-\Psi_{i+1} \Sigma_{i+1} \nabla K\left(x^{i}\right) \breve{P}_{i}\right]\left(x^{i+1}-\widehat{x}\right), y^{i+1}-\widehat{y}\right\rangle .
\end{aligned}
$$

Defining for brevity

$$
\begin{aligned}
& k_{\ell, j}:=\left\langle\nabla K\left(x^{i}\right)^{*}\left(y_{\ell}^{i+1}-\widehat{y}_{\ell}\right), x_{j}^{i+1}-\widehat{x}_{j}\right\rangle \text { and } \\
& k_{\ell, j}^{+}:=\left\langle\nabla K\left(x^{i+1}\right)^{*}\left(y_{\ell}^{i+1}-\widehat{y}_{\ell}\right), x_{j}^{i+1}-\widehat{x}_{j}\right\rangle
\end{aligned}
$$

and using (3.14), which implies $\phi_{j}^{i} \tau_{j}^{i}, \psi_{\ell}^{i+1} \sigma_{\ell}^{i+1} \in \mathcal{R}\left(\mathcal{O}_{i} ;(0, \infty)\right)$, we expand

$$
\begin{aligned}
\mathbb{E}_{i}\left[D_{i}^{\Lambda}\right]=\sum_{\ell=1}^{n} \sum_{j=1}^{m} & {\left[\left(\chi_{S(i)}(j) \phi_{j}^{i} \tau_{j}^{i}-\chi_{V(i+1)}(\ell) \psi_{\ell}^{i+1} \sigma_{\ell}^{i+1}\right) k_{\ell, j}\right.} \\
+ & \mathbb{E}_{i}\left[\chi_{V(i+2)}(\ell)\left(\chi_{\dot{S}(i+1)}(j) \phi_{j}^{i+1} \stackrel{\tau}{j}^{i+1}-\chi_{S(i+1) \backslash \grave{S}(i+1)}(j) \psi_{\ell}^{i+2} \sigma_{\ell}^{i+2}\right) k_{\ell, j}^{+}\right] \\
& \left.-\chi_{V(i+1)}(\ell)\left(\chi_{\dot{S}(i)}(j) \phi_{j}^{i} \dot{\tau}_{j}^{i}-\chi_{S(i) \backslash \dot{S}(i)}(j) \psi_{\ell}^{i+1} \sigma_{\ell}^{i+1}\right) k_{\ell, j}\right] .
\end{aligned}
$$


Writing in the first term $\chi_{S(i)}(j) \phi_{j}^{i} \tau_{j}^{i}=\chi_{\dot{S}(i)}(j) \phi_{j}^{i} \stackrel{\circ}{i}_{j}^{i}+\chi_{S(i) \backslash \dot{S}(i)}(j) \phi_{j}^{i} \breve{\tau}_{j}^{i}$, this rearranges as

$$
\begin{aligned}
\mathbb{E}_{i}\left[D_{i}^{\Lambda}\right]=\sum_{\ell=1}^{n} \sum_{j=1}^{m}\left(\left[\chi_{S(i) \backslash \grave{S}(i)}(j) \phi_{j}^{i} \breve{\tau}_{j}^{i}+\left(1-\chi_{V(i+1)}(\ell)\right) \chi_{\dot{S}(i)}(j) \phi_{j}^{i} \dot{\tau}_{j}^{i}\right.\right. \\
\left.+\chi_{V(i+1)}(\ell)\left(\chi_{S(i) \backslash \dot{S}(i)}(j)-1\right) \psi_{\ell}^{i+1} \sigma_{\ell}^{i+1}\right] k_{\ell, j} \\
+\mathbb{E}_{i}\left[\chi_{V(i+2)}(\ell) \chi_{\dot{S}(i+1)}(j) \phi_{j}^{i+1} \dot{\tau}_{j}^{i+1}\right. \\
\left.\left.-\chi_{V(i+2)}(\ell) \chi_{S(i+1) \backslash \stackrel{S}{S}(i+1)}(j) \psi_{\ell}^{i+2} \sigma_{\ell}^{i+2}\right] k_{\ell, j}^{+}\right) .
\end{aligned}
$$

Using (2.9), we continue

$$
\begin{aligned}
\mathbb{E}_{i}\left[D_{i}^{\Lambda}\right]=\sum_{\ell=1}^{n} \sum_{j=1}^{m}( & {\left[\chi_{S(i) \backslash \grave{S}(i)}(j) \phi_{j}^{i} \breve{\tau}_{j}^{i}-\chi_{V(i+1) \backslash \dot{V}(i+1)}(\ell) \psi_{\ell}^{i+1} \breve{\sigma}_{\ell}^{i+1}\right] k_{\ell, j} } \\
& \left.+\mathbb{E}_{i}\left[\chi_{\dot{S}(i+1)}(j) \phi_{j}^{i+1} \tilde{\tau}_{j}^{i+1}-\chi_{\dot{V}(i+2)}(\ell) \psi_{\ell}^{i+2} \stackrel{\circ}{\sigma}_{\ell}^{i+2}\right] k_{\ell, j}^{+}\right)
\end{aligned}
$$

after which a use of (3.19) rearranges this as

$$
\begin{aligned}
\mathbb{E}_{i}\left[D_{i}^{\Lambda}\right] & =\sum_{\ell=1}^{n} \sum_{j=1}^{m}\left(\stackrel{\circ}{\pi}_{j}^{i+1} \phi_{j}^{i+1} \stackrel{\circ}{\tau}_{j}^{i+1}-\stackrel{\circ}{\nu}_{\ell}^{i+2} \psi_{\ell}^{i+2} \stackrel{\circ}{\sigma}_{\ell}^{i+2}\right)\left(k_{\ell, j}^{+}-k_{\ell, j}\right) \\
& =\sum_{\ell=1}^{n} \sum_{j=1}^{m}\left(\chi_{S(i) \backslash \grave{S}(i)}(j) \phi_{j}^{i} \breve{\tau}_{j}^{i}-\chi_{V(i+1) \backslash \grave{V}(i+1)}(\ell) \psi_{\ell}^{i+1} \breve{\sigma}_{\ell}^{i+1}\right)\left(k_{\ell, j}-k_{\ell, j}^{+}\right) .
\end{aligned}
$$

Expanding $k_{\ell, j}-k_{\ell, j}^{+}$, using Assumption 3.1, and continuing with Young's inequality yields for any $\alpha_{x}, \alpha_{y}>0$,

$$
\begin{aligned}
\mathbb{E}_{i}\left[D_{i}^{\Lambda}\right]= & \sum_{\ell=1}^{n} \sum_{j=1}^{m}\left[\left(\chi_{S(i) \backslash \dot{S}(i)}(j) \phi_{j}^{i} \breve{\tau}_{j}^{i}-\chi_{V(i+1) \backslash \dot{V}(i+1)}(\ell) \psi_{\ell}^{i+1} \breve{\sigma}_{\ell}^{i+1}\right)\right. \\
& \left.\cdot\left\langle y_{\ell}^{i+1}-\widehat{y}_{\ell},\left[\nabla K\left(x^{i}\right)-\nabla K\left(x^{i+1}\right)\right]\left(x_{j}^{i+1}-\widehat{x}_{j}\right)\right\rangle\right] \\
\geq & -\sum_{j=1}^{m} \chi_{S(i) \backslash \grave{S}(i)}(j) \phi_{j}^{i} \breve{\tau}_{j}^{i} \cdot\left\|y^{i+1}-\widehat{y}\right\|_{P_{\mathrm{NL}}} L\left\|x^{i+1}-x^{i}\right\|\left\|x_{j}^{i+1}-\widehat{x}_{j}\right\| \\
& -\sum_{\ell=1}^{n} \chi_{V(i+1) \backslash \grave{V}(i+1)}(\ell) \psi_{\ell}^{i+1} \breve{\sigma}_{\ell}^{i+1} \cdot\left\|y_{\ell}^{i+1}-\widehat{y}_{\ell}\right\|_{P_{\mathrm{NL}}} L\left\|x^{i+1}-x^{i}\right\|\left\|x^{i+1}-\widehat{x}\right\| \\
\geq & -\sum_{j=1}^{m} \chi_{S(i) \backslash \dot{S}(i)}(j) \phi_{j}^{i} \breve{\tau}_{j}^{i}\left(\alpha_{x}\left\|x_{j}^{i+1}-\widehat{x}_{j}\right\|^{2}+\frac{L^{2}}{4 \alpha_{x}}\left\|y^{i+1}-\widehat{y}\right\|_{P_{\mathrm{NL}}}^{2}\left\|x^{i+1}-x^{i}\right\|^{2}\right) \\
& -\sum_{\ell=1}^{n} \chi_{V(i+1) \backslash \dot{V}(i+1)}(\ell) \psi_{\ell}^{i+1} \breve{\sigma}_{\ell}^{i+1}\left(\alpha_{y}\left\|y_{\ell}^{i+1}-\widehat{y}_{\ell}\right\|_{P_{\mathrm{NL}}}^{2}\right. \\
& \left.+\frac{L^{2}}{4 \alpha_{y}}\left\|x^{i+1}-x^{i}\right\|^{2}\left\|x^{i+1}-\widehat{x}\right\|^{2}\right) .
\end{aligned}
$$

This rearranges as (3.20). By (3.21), $\mathbb{P}\left[d^{i+1} \leq c_{*}^{i} \mid \mathcal{O}_{i-1}\right]=1$. Hence, (3.22) follows.

REMARK 3.10. For slightly stronger results, it would, in (3.21) and throughout the rest of the manuscript, be possible to take $\rho_{x}=\rho_{x}^{i+1}$ and $\rho_{\ell}=\rho_{\ell}^{i+1}$ dependent on the iteration. 
3.5. Putting it all together. We are now ready to state our main generic result providing the tool to estimate convergence rates based on growth rates of $\phi_{j}^{i}$ and $\psi_{\ell}^{i+1}$.

THEOREM 3.11. Suppose that Assumptions 3.1, 3.2, and 3.4 hold for some $0<\delta \leq \kappa<1$, $\gamma_{G, j}, \gamma_{F^{*}, \ell} \geq 0, \gamma_{K, j} \in \mathbb{R}(j=1, \ldots, m, \ell=1, \ldots, n), L, L_{3} \geq 0, p \in[1,2], \theta_{A} \geq 0$ together with the nesting conditions (2.9), the lower bound (3.6) for the local metric, and the conditionality assumptions (3.14), for all $i \leq N-1$. For some sequence of $\eta^{i+1}>0$ assume the coupling conditions

$$
\begin{aligned}
& \stackrel{\circ}{\pi}_{j}^{i+1} \phi_{j}^{i+1} \stackrel{\sim}{j}_{j}^{i+1}+\chi_{S(i) \backslash \stackrel{S}{S}(i)}(j) \phi_{j}^{i} \breve{\tau}_{j}^{i}=\eta^{i+1} \quad(j=1, \ldots, m) \quad \text { and } \\
& \stackrel{\circ}{\nu}_{\ell}^{i+2} \psi_{\ell}^{i+2} \stackrel{\circ}{\sigma}_{\ell}^{i+2}+\chi_{V(i+1) \backslash \dot{V}(i+1)}(\ell) \psi_{\ell}^{i+1} \breve{\sigma}_{\ell}^{i+1}=\eta^{i+1} \quad(\ell=1, \ldots, n) .
\end{aligned}
$$

Also assume for some $\rho_{x}, \rho_{\ell} \geq 0$ and $\zeta_{\ell} \geq 0$,

(3.25a) $\quad 1=\mathbb{P}\left[\left\|x^{i+1}-\widehat{x}\right\| \leq \rho_{x},\left\|Q_{\ell}\left(y^{i+1}-\widehat{y}\right)\right\|_{P_{\mathrm{NL}}} \leq \rho_{\ell},(\ell=1, \ldots, n) \mid \mathcal{O}_{i-1}\right]$ and

(3.25b) $\mathbb{E}_{i-1}\left[\theta_{\Phi_{i} T_{i}}\right] \geq p^{-p} \sum_{\ell=1}^{n} \nu_{\ell}^{i+1} \psi_{\ell}^{i+1} \sigma_{\ell}^{i+1} \zeta_{\ell}^{1-p} \rho_{\ell}^{2-p} \quad(\ell=1, \ldots, n)$.

Finally, for $c_{*}^{i}$ defined in (3.23) for some $\alpha_{x}, \alpha_{y}>0$, let

$$
\begin{aligned}
L_{j}^{i} & :=L_{3}+\left(L\left\|\Omega^{i}+I\right\|^{2} \sum_{\ell=1}^{m} \psi_{\ell}^{i+1} \sigma_{\ell}^{i+1} \rho_{\ell}+c_{*}^{i}\right) / \phi_{j}^{i} \tau_{j}^{i}, \\
\bar{\gamma}_{G K, j}^{i} & :=\gamma_{G, j}+\gamma_{K, j}-\chi_{S(i) \backslash S(i)}(j) \alpha_{x}, \\
\bar{\gamma}_{F^{*}, \ell}^{i+1} & := \begin{cases}\gamma_{F^{*}, \ell}, & Q_{\ell} P_{\mathrm{NL}}=0, \\
\gamma_{F^{*}, \ell}-(p-1) \zeta_{\ell}-\chi_{V(i+1) \backslash \dot{V}(i+1)}(\ell) \alpha_{y}, & Q_{\ell} P_{\mathrm{NL}} \neq 0 .\end{cases}
\end{aligned}
$$

Then

$$
\begin{aligned}
\delta \sum_{j=1}^{m} \mathbb{E}\left[\phi_{j}^{i}\left\|P_{j}\left(x^{N}-\widehat{x}\right)\right\|^{2}\right]+\frac{\kappa-\delta}{1-\delta} \sum_{\ell=1}^{n} \mathbb{E}\left[\psi_{\ell}^{i+1}\left\|Q_{\ell}\left(y^{N}-\widehat{y}\right)\right\|^{2}\right] \\
\leq \mathbb{E}\left[\left\|u^{N}-\widehat{u}\right\|_{Z_{N+1} M_{N+1}}^{2}\right] \leq \mathbb{E}\left[\left\|u^{0}-\widehat{u}\right\|_{Z_{1} M_{1}}^{2}\right]
\end{aligned}
$$

holds provided that for every $i \leq N-1$ both (i) and (ii) are true:

(i) Either of the primal test update conditions holds for every $j=1, \ldots, m$ :

(a) both $\phi_{j}^{i+1} \leq\left(1+2 \chi_{S(i)}(j) \tau_{j}^{i} \bar{\gamma}_{G K, j}^{i}\right) \phi_{j}^{i}$ and $\delta \geq \chi_{S(i)}(j) L_{j}^{i} \tau_{j}^{i}$; or

(b) for some $\widetilde{\gamma}_{G, j}^{i} \in \mathcal{R}\left(\mathcal{O}_{i-1}, \mathbb{R}\right), \widetilde{\tau}_{j}^{i}:=\left(\stackrel{\circ}{\pi}_{j}^{i} \tilde{\tau}_{j}^{i}+\left(\pi_{j}^{i}-\stackrel{\circ}{\pi}_{j}^{i}\right) \breve{\tau}_{j}^{i}\right) / \pi_{j}^{i}$,

$$
\begin{aligned}
\phi_{j}^{i+1}= & \left(1+2 \widetilde{\tau}_{j}^{i} \widetilde{\gamma}_{G, j}^{i}\right) \phi_{j}^{i}, \quad \widetilde{\tau}_{j}^{i} \widetilde{\gamma}_{G, j}^{i}<\mathbb{E}_{i-1}\left[\chi_{S(i)}(j) \tau_{j}^{i} \bar{\gamma}_{G K, j}^{i}\right], \text { and } \\
\delta \geq & \chi_{S(i)}(j) L_{j}^{i} \tau_{j}^{i} \\
& +\chi_{S(i)}(j) \frac{2\left(\tau_{j}^{i} \bar{\gamma}_{G K, j}^{i}-\mathbb{E}_{i-1}\left[\chi_{S(i)}(j) \tau_{j}^{i} \bar{\gamma}_{G K, j}^{i}\right]\right)\left(\tau_{j}^{i} \bar{\gamma}_{G K, j}^{i}-\widetilde{\tau}_{j}^{i} \widetilde{\gamma}_{G, j}^{i}\right)}{\mathbb{E}_{i-1}\left[\chi_{S(i)}(j) \tau_{j}^{i} \bar{\gamma}_{G K, j}^{i}\right]-\widetilde{\tau}_{j}^{i} \widetilde{\gamma}_{G, j}^{i}}
\end{aligned}
$$

(ii) Either of the dual test update conditions holds for every $\ell=1, \ldots, n$ :

(a) $\psi_{\ell}^{i+1} \leq\left(1+2 \chi_{V(i+1)}(\ell) \sigma_{\ell}^{i+1} \bar{\gamma}_{F^{*}, \ell}^{i+1}\right) \psi_{\ell}^{i+1}$; or

(b) for some $\widetilde{\gamma}_{F^{*}, \ell}^{i+1} \in \mathcal{R}\left(\mathcal{O}_{i-1}, \mathbb{R}\right), \widetilde{\sigma}_{\ell}^{i+1}:=\left({ }_{\nu}{ }_{\ell}^{i+1} \stackrel{\circ}{\sigma}_{\ell}^{i+1}+\left(\nu_{\ell}^{i+1}-\stackrel{\circ}{\nu}_{\ell}^{i+1}\right) \breve{\sigma}_{\ell}^{i+1}\right) / \nu_{\ell}^{i+1}$ : 


$$
\begin{aligned}
& \text { (3.31a) } \psi_{\ell}^{i+2}=\left(1+2 \widetilde{\sigma}_{\ell}^{i+1} \widetilde{\gamma}_{F^{*}, \ell}^{i+1}\right) \psi_{\ell}^{i+1}, \\
& \text { (3.31b) } \widetilde{\sigma}_{\ell}^{i+1} \widetilde{\gamma}_{F^{*}, \ell}^{i+1}< \mathbb{E}_{i-1}\left[\chi_{V(i+1)}(\ell) \sigma_{\ell}^{i+1} \bar{\gamma}_{F^{*}, \ell}^{i+1}\right], \\
& \text { (3.31c) } \frac{\kappa-\delta}{1-\delta} \geq 2\left(\sigma_{\ell}^{i+1} \bar{\gamma}_{F^{*}, \ell}^{i+1}-\mathbb{E}_{i-1}\left[\chi_{V(i+1)}(\ell) \sigma_{\ell}^{i+1} \bar{\gamma}_{F^{*}, \ell}^{i+1}\right]\right) \\
& \cdot \frac{\chi_{V(i+1)}(\ell)\left(\sigma_{\ell}^{i+1} \bar{\gamma}_{F^{*}, \ell}^{i+1}-\widetilde{\sigma}_{\ell}^{i+1} \widetilde{\gamma}_{F^{*}, \ell}^{i+1}\right)}{\mathbb{E}_{i-1}\left[\chi_{V(i+1)}(\ell) \sigma_{\ell}^{i+1} \bar{\gamma}_{F^{*}, \ell}^{i+1}\right]-\widetilde{\sigma}_{\ell}^{i+1} \widetilde{\gamma}_{F^{*}, \ell}^{i+1}} .
\end{aligned}
$$

Proof. We first apply Theorem 3.7. Recalling $R^{\prime}$ from (3.8b), let us set (3.32)

$R^{\prime \prime}:=R^{\prime}$

$$
\begin{aligned}
& -2\left[\begin{array}{cc}
\sum_{j=1}^{m} \tau_{j}^{i} \phi_{j}^{i} \chi_{S(i) \backslash \hat{S}(i)}(j) \alpha_{x} P_{j} & 0 \\
0 & \sum_{\ell=1}^{n} \sigma_{\ell}^{i+1} \psi_{\ell}^{i+1}\left(\chi_{V(i+1)}(\ell)(p-1) \zeta_{\ell}+\chi_{V(i+1) \backslash \dot{V}(i+1)}(\ell) \alpha_{y}\right) Q_{\ell} P_{\mathrm{NL}}
\end{array}\right] \\
= & {\left[\begin{array}{cc}
\Phi_{i}-\Phi_{i+1}+2 \sum_{j \in S(i)} \phi_{j}^{i} \tau_{j}^{i} \bar{\gamma}_{G K, j} P_{j} & 0 \\
0 & \Psi_{i+1}-\Psi_{i+2}+2 \sum_{\ell \in V(i+1)} \psi_{\ell}^{i+1} \sigma_{\ell}^{i+1} \bar{\gamma}_{F^{*}, \ell} Q_{\ell}
\end{array}\right] } \\
= & {\left[\begin{array}{cc}
\sum_{j=1}^{m} q_{j}^{i} P_{j} & 0 \\
0 & \sum_{\ell=1}^{n} h_{\ell}^{i+1} Q_{\ell}
\end{array}\right] }
\end{aligned}
$$

for

$$
\begin{aligned}
q_{j}^{i} & :=\left(1+2 \chi_{S(i)}(j) \tau_{j}^{i} \bar{\gamma}_{G K, j}^{i}\right) \phi_{j}^{i}-\phi_{j}^{i+1} \quad \text { and } \\
h_{\ell}^{i+1} & :=\left(1+2 \chi_{V(i+1)}(\ell) \sigma_{\ell}^{i+1} \bar{\gamma}_{F^{*}, \ell}^{i+1}\right) \psi_{\ell}^{i+1}-\psi_{\ell}^{i+2} .
\end{aligned}
$$

Thus,

$$
\begin{aligned}
\mathbb{E}_{i-1}\left[\| u^{i+1}\right. & \left.-\widehat{u} \|_{R^{\prime \prime}}^{2}\right] \\
& =\sum_{j=1}^{m} \mathbb{E}_{i-1}\left[q_{j}^{i}\left\|P_{j}\left(x^{i+1}-\widehat{x}\right)\right\|^{2}\right]+\sum_{\ell=1}^{n} \mathbb{E}_{i-1}\left[h_{\ell}^{i+1}\left\|Q_{\ell}\left(y^{i+1}-\widehat{y}\right)\right\|^{2}\right] .
\end{aligned}
$$

Estimation of $q_{j}^{i}$. Suppose that $j \in\{1, \ldots, m\}$ satisfies (i)(a). Then $q_{j}^{i} \geq 0$ and $\delta \geq \chi_{S(i)}(j) L_{j}^{i} \tau_{j}^{i}$, so we immediately estimate

$$
\mathbb{E}_{i-1}\left[q_{j}^{i}\left\|P_{j}\left(x^{i+1}-\widehat{x}\right)\right\|^{2}\right] \geq-\mathbb{E}_{i-1}\left[\chi_{S(i)}(j)\left(\delta \phi_{j}^{i}-L_{j}^{i} \phi_{j}^{i} \tau_{j}^{i}\right)\left\|P_{j}\left(x^{i+1}-x^{i}\right)\right\|^{2}\right] .
$$

Otherwise, if $j \in\{1, \ldots, m\}$ satisfies (i)(b), using (3.15) and that $q_{j}^{i}=\mathbb{E}_{i}\left[q_{j}^{i}\right]$ due to (3.14) and (3.27), we decompose

$$
\begin{aligned}
\mathbb{E}_{i-1}\left[q_{j}^{i}\left\|P_{j}\left(x^{i+1}-\widehat{x}\right)\right\|^{2}\right]=\mathbb{E}_{i-1}[ & q_{j}^{i}\left\|P_{j}\left(x^{i+1}-x^{i}\right)\right\|^{2}+\mathbb{E}_{i-1}\left[q_{j}^{i}\right]\left\|P_{j}\left(x^{i}-\widehat{x}\right)\right\|^{2} \\
& \left.+2 q_{j}^{i}\left\langle P_{j}\left(x^{i+1}-x^{i}\right), x^{i}-\widehat{x}\right\rangle\right]
\end{aligned}
$$

Using $\left(1-\chi_{S(i)}(j)\right) P_{j}\left(x^{i+1}-x^{i}\right)=0$ and Young's inequality with the factor $\alpha>0$, we obtain

$$
\begin{aligned}
& \mathbb{E}_{i-1}\left[q_{j}^{i}\left\|P_{j}\left(x^{i+1}-\widehat{x}\right)\right\|^{2}\right] \\
& \geq \mathbb{E}_{i-1}\left[\chi_{S(i)}(j)\left(q_{j}^{i}-\alpha\left|q_{j}^{i}\right|\right)\left\|P_{j}\left(x^{i+1}-x^{i}\right)\right\|^{2}\right. \\
& \left.\quad+\left(\mathbb{E}_{i-1}\left[q_{j}^{i}\right]-\chi_{S(i)}(j) \alpha^{-1}\left|q_{j}^{i}\right|\right)\left\|P_{j}\left(x^{i}-\widehat{x}\right)\right\|^{2}\right]
\end{aligned}
$$


Since $\phi_{j}^{i+1}=\left(1+2 \widetilde{\tau}_{j}^{i} \widetilde{\gamma}_{G, j}^{i}\right) \phi_{j}^{i}$ with $\widetilde{\gamma}_{G, j}^{i} \in \mathcal{R}\left(\mathcal{O}_{i-1} ; \mathbb{R}\right)$, we have from (3.30a)

$$
\begin{aligned}
\mathbb{E}_{i-1}\left[q_{j}^{i}\right] & =\left(1+2 \mathbb{E}_{i-1}\left[\chi_{S(i)}(j) \tau_{j}^{i} \bar{\gamma}_{G K, j}^{i}\right]\right) \phi_{j}^{i}-\mathbb{E}_{i-1}\left[\phi_{j}^{i+1}\right] \\
& =2 \phi_{j}^{i}\left(\mathbb{E}\left[\chi_{S(i)}(j) \tau_{j}^{i} \bar{\gamma}_{G K, j}^{i}\right]-\widetilde{\tau}_{j}^{i} \widetilde{\gamma}_{G, j}^{i}\right)>0,
\end{aligned}
$$

and rearranging (3.30b) for $j \in S(i)$ yields

$$
q_{j}^{i}=2 \phi_{j}^{i}\left(\chi_{S(i)}(j) \tau_{j}^{i} \bar{\gamma}_{G K, j}^{i}-\widetilde{\tau}_{j}^{i} \widetilde{\gamma}_{G, j}^{i}\right) \geq\left(\mathbb{E}_{i-1}\left[q_{j}^{i}\right]\right)^{-1}\left|q_{j}^{i}\right|^{2}-\delta \phi_{j}^{i}+L_{j}^{i} \phi_{j}^{i} \tau_{j}^{i}
$$

Therefore, taking $\alpha:=\left(\mathbb{E}_{i-1}\left[q_{j}^{i}\right]\right)^{-1}\left|q_{j}^{i}\right|$ for $j \in S(i)$ in (3.35), we verify (3.34) for the case (i)(b) as well.

Estimation of $h_{\ell}^{i+1}$. Similarly, if $\ell \in\{1, \ldots, n\}$ satisfies (ii)(a), then we have $h_{\ell}^{i+1} \geq 0$, hence

$$
\mathbb{E}_{i-1}\left[h_{\ell}^{i+1}\left\|Q_{\ell}\left(y^{i+1}-\widehat{y}\right)\right\|^{2}\right] \geq-\mathbb{E}_{i-1}\left[\chi_{V(i+1)}(\ell) \frac{\kappa-\delta}{1-\delta} \psi_{\ell}^{i+1}\left\|Q_{\ell}\left(y^{i+1}-y^{i}\right)\right\|^{2}\right] .
$$

Otherwise, when $\ell \in\{1, \ldots, n\}$ satisfies (ii)(b), using (3.15) and that $h_{\ell}^{i+1}=\mathbb{E}_{i}\left[h_{\ell}^{i+1}\right]$ due to (3.14) and (3.28), we estimate for arbitrary $\alpha>0$ that

$$
\begin{aligned}
& \mathbb{E}_{i-1}\left[h_{\ell}^{i+1}\left\|Q_{\ell}\left(y^{i+1}-\widehat{y}\right)\right\|^{2}\right] \\
& \geq \mathbb{E}_{i-1}\left[\chi_{V(i+1)}(\ell)\left(h_{\ell}^{i+1}-\alpha\left|h_{\ell}^{i+1}\right|\right)\left\|Q_{\ell}\left(y^{i+1}-y^{i}\right)\right\|^{2}\right. \\
& \left.\quad+\left(\mathbb{E}_{i-1}\left[h_{\ell}^{i+1}\right]-\chi_{V(i+1)}(\ell) \alpha^{-1}\left|h_{\ell}^{i+1}\right|\right)\left\|Q_{\ell}\left(y^{i}-\widehat{y}\right)\right\|^{2}\right] .
\end{aligned}
$$

Since $\psi_{\ell}^{i+2}=\left(1+2 \widetilde{\sigma}_{\ell}^{i+1} \widetilde{\gamma}_{F^{*}, \ell}^{i+1}\right) \psi_{\ell}^{i+1}$ with $\widetilde{\gamma}_{F^{*}, \ell}^{i+1} \in \mathcal{R}\left(\mathcal{O}_{i-1} ; \mathbb{R}\right)$, from (3.31b) we have

$$
\mathbb{E}_{i-1}\left[h_{\ell}^{i+1}\right]=\left(1+2 \mathbb{E}_{i-1}\left[\chi_{V(i+1)}(\ell) \sigma_{\ell}^{i+1} \bar{\gamma}_{F^{*}, \ell}^{i+1}\right]\right) \psi_{\ell}^{i+1}-\mathbb{E}_{i-1}\left[\psi_{\ell}^{i+2}\right]>0,
$$

and rearranging (3.31c) for $\ell \in V(i+1)$ yields

$$
h_{\ell}^{i+1} \geq\left(\mathbb{E}_{i-1}\left[h_{\ell}^{i+1}\right]\right)^{-1}\left|h_{\ell}^{i+1}\right|^{2}-\frac{\kappa-\delta}{1-\delta} \psi_{\ell}^{i+1}
$$

Consequently, taking $\alpha:=\left(\mathbb{E}_{i-1}\left[h_{\ell}^{i+1}\right]\right)^{-1}\left|h_{\ell}^{i+1}\right|$ for $\ell \in V(i+1)$ in (3.37), we obtain (3.36) for the case (ii)(b) as well.

Combining the estimates. Since (3.34) and (3.36) hold for all $j=1, \ldots, m$ and $\ell=1, \ldots, n$, respectively, continuing from (3.33), we get

$$
\begin{aligned}
\mathbb{E}_{i-1}\left[\left\|u^{i+1}-\widehat{u}\right\|_{R^{\prime \prime}}^{2}\right] \geq-\mathbb{E}_{i-1}\left[\sum_{j=1}^{m}\right. & \left(\chi_{S(i)}(j)\left(\delta \phi_{j}^{i}-L_{j}^{i} \phi_{j}^{i} \tau_{j}^{i}\right)\left\|P_{j}\left(x^{i+1}-x^{i}\right)\right\|^{2}\right. \\
& \left.+\sum_{\ell=1}^{n}\left(\chi_{V(i+1)}(\ell) \frac{\kappa-\delta}{1-\delta} \psi_{\ell}^{i+1}\left\|Q_{\ell}\left(y^{i+1}-y^{i}\right)\right\|^{2}\right)\right] .
\end{aligned}
$$


Plugging in $L_{j}^{i}$ from (3.26) gives

$$
\begin{aligned}
& \mathbb{E}_{i-1}\left[\left\|u^{i+1}-\widehat{u}\right\|_{R^{\prime \prime}}^{2}\right] \\
& \geq-\mathbb{E}_{i-1}\left[\sum_{\ell=1}^{n}\left(\chi_{V(i+1)}(\ell) \frac{\kappa-\delta}{1-\delta} \psi_{\ell}^{i+1}\left\|Q_{\ell}\left(y^{i+1}-y^{i}\right)\right\|^{2}\right)\right. \\
& \quad+\sum_{j=1}^{m} \chi_{S(i)}(j)\left(\delta \phi_{j}^{i}-L\left\|\Omega^{i}+I\right\|^{2} \sum_{\ell=1}^{m} \psi_{\ell}^{i+1} \sigma_{\ell}^{i+1} \rho_{\ell}\right)\left\|P_{j}\left(x^{i+1}-x^{i}\right)\right\|^{2} \\
& \quad-\sum_{j=1}^{m}\left(\chi_{S(i)}(j)\left(L_{3} \phi_{j}^{i} \tau_{j}^{i}+c_{*}^{i}\right)\left\|P_{j}\left(x^{i+1}-x^{i}\right)\right\|^{2}\right]
\end{aligned}
$$

By the definitions of $R_{x}$ in (3.8) and $\rho_{\ell}$ in (3.25a), we continue with

$$
\begin{aligned}
\mathbb{E}_{i-1}\left[\left\|u^{i+1}-\widehat{u}\right\|_{R^{\prime \prime}}^{2}\right] \geq-\mathbb{E}_{i-1}[ & {\left[\left\|x^{i+1}-x^{i}\right\|_{R_{x}}^{2}+\frac{\kappa-\delta}{1-\delta}\left\|y^{i+1}-y^{i}\right\|_{\Psi_{i+1}}^{2}\right.} \\
& \left.-\sum_{j=1}^{m} \chi_{S(i)}(j)\left(L_{3} \phi_{j}^{i} \tau_{j}^{i}+c_{*}^{i}\right)\left\|P_{j}\left(x^{i+1}-x^{i}\right)\right\|^{2}\right]
\end{aligned}
$$

On the other hand, by the definition of $R^{\prime \prime}$ in (3.32),

$$
\begin{aligned}
\mathbb{E}_{i-1} & {\left[\left\|u^{i+1}-\widehat{u}\right\|_{R^{\prime \prime}}^{2}\right] } \\
= & \mathbb{E}_{i-1}\left[\left\|u^{i+1}-\widehat{u}\right\|_{R^{\prime}}^{2}-2 \alpha_{x} \sum_{j=1}^{m} \tau_{j}^{i} \phi_{j}^{i} \chi_{S(i) \backslash S(i)}(j)\left\|P_{j}\left(x^{i+1}-\widehat{x}\right)\right\|^{2}\right. \\
& \left.-2 \sum_{\ell=1}^{n}\left(\chi_{V(i+1)}(\ell)(p-1) \zeta_{\ell}+\chi_{V(i+1) \backslash \dot{V}(i+1)}(\ell) \alpha_{y}\right) \sigma_{\ell}^{i+1} \psi_{\ell}^{i+1}\left\|Q_{\ell}\left(y^{i+1}-\widehat{y}\right)\right\|_{P_{\mathrm{NL}}}^{2}\right] .
\end{aligned}
$$

Combining with (3.38) and rearranging the terms, we therefore have

$$
\mathbb{E}_{i-1}\left[\left\|u^{i+1}-\widehat{u}\right\|_{R^{\prime}}^{2}+\left\|x^{i+1}-x^{i}\right\|_{R_{x}}^{2}+\frac{\kappa-\delta}{1-\delta}\left\|y^{i+1}-y^{i}\right\|_{\Psi_{i+1}}^{2}\right] \geq \mathbb{E}_{i-1}\left[b_{1}+b_{2}\right]
$$

for

$$
\begin{aligned}
b_{1}:=\sum_{j=1}^{n} \chi_{S(i)}(j) L_{3} \phi_{j}^{i} \tau_{j}^{i}\left\|P_{j}\left(x^{i+1}-x^{i}\right)\right\|^{2} \\
\quad+2 \sum_{\ell=1}^{n} \sigma_{\ell}^{i+1} \psi_{\ell}^{i+1} \chi_{V(i+1)}(\ell)(p-1) \zeta_{\ell}\left\|Q_{\ell}\left(y^{i+1}-\widehat{y}\right)\right\|_{P_{\mathrm{NL}}}^{2}
\end{aligned}
$$

and

$$
\begin{aligned}
b_{2}:=2 \alpha_{x} & \sum_{j=1}^{m} \tau_{j}^{i} \phi_{j}^{i} \chi_{S(i) \backslash \stackrel{\leftrightarrow}{S}(i)}(j)\left\|P_{j}\left(x^{i+1}-\widehat{x}\right)\right\|^{2} \\
& +2 \alpha_{y} \sum_{\ell=1}^{n} \sigma_{\ell}^{i+1} \psi_{\ell}^{i+1} \chi_{V(i+1) \backslash \dot{V}(i+1)}(\ell)\left\|Q_{\ell}\left(y^{i+1}-\widehat{y}\right)\right\|_{P_{\mathrm{NL}}}^{2} \\
& +\sum_{j=1}^{n} \chi_{S(i)}(j) c_{*}^{i}\left\|P_{j}\left(x^{i+1}-x^{i}\right)\right\|^{2} .
\end{aligned}
$$


Our conditions (3.25) and $\delta \geq \chi_{S(i)}(j) L_{j}^{i} \tau_{j}^{i}$ ensure the conditions of Theorems 3.8 and 3.9. By Theorem 3.8, $\mathbb{E}_{i-1}\left[b_{1}+2 D_{i}^{K}\right] \geq 0$, while using both (3.20) and (3.22) of Theorem 3.9 establishes $\mathbb{E}_{i-1}\left[b_{2}+2 D_{i}^{\Lambda}\right]=\mathbb{E}_{i-1}\left[b_{2}+2 \mathbb{E}_{i}\left[D_{i}^{\Lambda}\right]\right] \geq 0$. Consequently, (3.39) yields

$$
\mathbb{E}_{i-1}\left[\left\|u^{i+1}-\widehat{u}\right\|_{R^{\prime}}^{2}+\left\|x^{i+1}-x^{i}\right\|_{R_{x}}^{2}+\frac{\kappa-\delta}{1-\delta}\left\|y^{i+1}-y^{i}\right\|_{\Psi_{i+1}}^{2}+2 D_{i}^{\Lambda}+2 D_{i}^{K}\right] \geq 0 .
$$

We now use Theorem 3.7 to verify (2.5). Minding that each $Z_{i+1} M_{i+1}$ is self-adjoint by Theorem 3.5, a referral to Theorem 2.1 establishes (2.6). Using (3.6) as well as the properties $\phi_{j}^{N}, \psi_{\ell}^{N+1} \in \mathcal{R}\left(\mathcal{O}_{N-1} ;(0, \infty)\right)$ and $u^{N} \in \mathcal{R}\left(\mathcal{O}_{N-1} ; X \times Y\right)$ that follow from (3.14), we estimate

$$
\begin{aligned}
& \mathbb{E}\left[\left\|u^{N}-\widehat{u}\right\|_{Z_{N+1} M_{N+1}}^{2}\right.\left.\mid \mathcal{O}_{N-1}\right]=\left\|u^{N}-\widehat{u}\right\|_{\mathbb{E}\left[Z_{N+1} M_{N+1} \mid \mathcal{O}_{N-1}\right]}^{2} \\
& \geq \delta \sum_{j=1}^{m} \phi_{j}^{N}\left\|P_{j}\left(x^{N}-\widehat{x}\right)\right\|^{2}+\frac{\kappa-\delta}{1-\delta} \sum_{\ell=1}^{n} \psi_{\ell}^{N+1}\left\|Q_{\ell}\left(y^{N}-\widehat{y}\right)\right\|^{2} .
\end{aligned}
$$

Taking the full expectation and using (2.6) establishes the claim.

REMARK 3.12. The conditions (i)(a) and (ii)(a) differ from (i)(b) and (ii)(b) by the larger factors $\bar{\gamma}_{G K, j}^{i}$ and $\bar{\gamma}_{F^{*}, \ell}^{i}$ and by updating $\phi_{j}^{i+1}$ and $\psi_{\ell}^{i+2} \in \mathcal{R}\left(\mathcal{O}_{i} ; \mathbb{R}\right)$ potentially non-deterministically.

In Section 4 we have $\stackrel{\circ}{i}_{j}^{i}=\pi_{j}^{i}, \tau_{j}^{i}=\stackrel{\circ}{\tau}_{j}^{i}, \stackrel{\circ}{\nu}_{\ell}^{i+1}=0$, and $\sigma_{\ell}^{i+1}=\breve{\sigma}_{\ell}^{i+1}$. In Section 5 we take $\stackrel{\circ}{\pi}_{j}^{i}=0, \tau_{j}^{i}=\breve{\tau}_{j}^{i}, \stackrel{\circ}{\nu}_{\ell}^{i+1}=\nu_{\ell}^{i+1}$, and $\sigma_{\ell}^{i+1}=\stackrel{\circ}{\sigma}_{\ell}^{i+1}$. Also (i)(b) and (ii)(b) then simplify, for $\widetilde{\gamma}_{G, j}^{i}<\pi_{j}^{i} \bar{\gamma}_{G K, j}^{i}$, to

$$
\begin{aligned}
\phi_{j}^{i+1} & =\left(1+2 \tau_{j}^{i} \widetilde{\gamma}_{G, j}^{i}\right) \phi_{j}^{i}, \\
\delta & \geq \chi_{S(i)}(j) \tau_{j}^{i}\left(L_{j}^{i}+2\left(1-\pi_{j}^{i}\right) \bar{\gamma}_{G K, j}^{i} \frac{\bar{\gamma}_{G K, j}^{i}-\widetilde{\gamma}_{G, j}^{i}}{\pi_{j}^{i} \bar{\gamma}_{G K, j}^{i}-\widetilde{\gamma}_{G, j}^{i}}\right)
\end{aligned}
$$

and, respectively, for $\widetilde{\gamma}_{F^{*}, \ell}^{i+1}<\nu_{\ell}^{i+1} \bar{\gamma}_{F^{*}, \ell}^{i+1}$, to

$$
\begin{aligned}
& \psi_{\ell}^{i+2}=\left(1+2 \sigma_{\ell}^{i+1} \widetilde{\gamma}_{F^{*}, \ell}^{i+1}\right) \psi_{\ell}^{i+1} \text { and } \\
& \frac{\kappa-\delta}{1-\delta} \geq 2 \chi_{V(i+1)}(\ell)\left(1-\nu_{\ell}^{i+1}\right) \sigma_{\ell}^{i+1} \bar{\gamma}_{F^{*}, \ell}^{i+1} \frac{\bar{\gamma}_{F^{*}, \ell}^{i+1}-\widetilde{\gamma}_{F^{*}, \ell}^{i+1}}{\nu_{\ell}^{i+1} \bar{\gamma}_{F^{*}, \ell}^{i+1}-\widetilde{\gamma}_{F^{*}, \ell}^{i+1}}
\end{aligned}
$$

REMARK 3.13. Another quite restrictive requirement that we will need in the next sections is the almost sure boundedness of the iterates in (3.25a). We already had this requirement in the deterministic single-block algorithm in [9, Section 4.3] and [10, Section 5]. We have verified in [9, Proposition 4.8.] that this requirement can be restated in terms of a sufficiently close initialisation of the iterations to the critical point, which is often required in non-convex optimisation.

In this work, the rates for convergence are in expectation, hence, the required boundedness is stated in almost sure terms. Moreover, in order to be able to update only some primal blocks in each iteration, (3.25a) now also requires the primal variable to be bounded. Through the simplified algorithms of Sections 4 and 5, treating respective non-randomised dual updates and non-randomised primal updates, we will somewhat relax these restrictions:

- Algorithm 4.2 of Section 4 will not require the dual variable to be bounded if Assumption 3.2 holds with $p=2$; see Theorems 4.6 and 4.8.

- In Section 5, we will not require any bound for the primal variable. 
In some cases, boundedness can, moreover, be verified analytically based on the explicit formula for $F$. For example, for $F(x)=|\langle a, x\rangle|$ or $F(x)=\|a x\|$, the support of $F^{*}(y)$ is bounded by $\|a\|$. Hence the range of the corresponding proximal operator is also bounded. In particular, if $F$ is of such a form, then the boundedness assumptions of Section 5 are automatically satisfied.

4. Methods with full dual updates. We now develop more specific methods based on (2.13) and study their convergence based on Theorem 3.11. In this section we take $\stackrel{\circ}{V}(i+1)=\emptyset, V(i+1)=\{1, \ldots, n\}$, and $\stackrel{\circ}{S}(i)=S(i)$ for all iterations $i$. The nesting conditions (2.9) of Theorem 3.11 then hold, and the coupling conditions (3.24) become

$$
\stackrel{\pi}{\pi}_{j}^{i+1} \phi_{j}^{i+1} \stackrel{\tau}{j}_{j}^{i+1}=\eta^{i+1}=\psi_{\ell}^{i+1} \breve{\sigma}_{\ell}^{i+1} .
$$

The dual update of (2.13) involves $\Psi_{i+1}^{-1}\left[\nabla K\left(x^{i}\right) T_{i}^{*} \Phi_{i}^{*}-\Psi_{i+1} \Sigma_{i+1} \nabla K\left(x^{i}\right) \Omega_{i}\right]$ in scalar form,

$$
\frac{\phi_{j}^{i} \dot{\tau}_{j}^{i}-\omega_{j}^{i} \breve{\sigma}_{\ell}^{i+1} \psi_{\ell}^{i+1}}{\psi_{\ell}^{i+1}}=\breve{\sigma}_{\ell}^{i+1}\left(\frac{\eta^{i}}{\pi_{j}^{i} \eta^{i+1}}-\omega_{j}^{i}\right)=\breve{\sigma}_{\ell}^{i+1}\left(\frac{\bar{\omega}^{i}}{\bar{\pi}_{j}^{i}}-\omega_{j}^{i}\right) \quad \text { for } \quad \bar{\omega}^{i}:=\frac{\eta^{i}}{\eta^{i+1}} .
$$

Therefore, with $\omega_{j}^{i}=\frac{\bar{\omega}^{i}}{\bar{\pi}_{j}^{i}}$, the updates (2.13) simplify to those of Algorithm 4.1. Moreover, (2.12) reduces to $\lambda_{j, \ell}^{i}=\phi_{j}^{i} \tau_{j}^{i} \chi_{S(i)}(j)$. We thus verify (3.6) via the following lemma:

Lemma 4.1. Suppose that $\stackrel{\circ}{V}(i+1)=\emptyset, V(i+1)=\{1, \ldots, n\}, \stackrel{\circ}{S}(i)=S(i)$ for $i \in \mathbb{N}$, the coupling condition (4.1) holds, and $\bar{\omega}^{i} \leq 1$. Moroever, suppose that for all $\ell=1, \ldots, n$ and $j=1, \ldots, m$,

$$
\bar{\omega}^{i} \breve{\sigma}_{\ell}^{i+1} \tilde{\tau}_{j}^{i} \leq \breve{\sigma}_{\ell}^{0} \stackrel{\tau}{j}_{j}^{0} \quad \text { and } \quad 1-\kappa \geq\left\|\sum_{j \in \dot{S}(i)} \sqrt{\frac{w_{j, \ell}^{i} \breve{\sigma}_{\ell}^{0} \tilde{\tau}_{j}^{0}}{\dot{\pi}_{j}^{i}}} Q_{\ell} \nabla K\left(x^{i}\right) P_{j}\right\|^{2}
$$

for some $0 \leq \kappa \leq 1$ and $w_{j, \ell, k}=1 / w_{j, k, \ell}>0$ such that

$$
w_{j, \ell}^{i}:=\chi_{\mathcal{V}_{j}^{i}}(\ell) \sum_{k \in \overline{\mathcal{V}}_{j}^{i}(\ell)} w_{j, \ell, k}
$$

with

$$
\overline{\mathcal{V}}_{j}^{i}(\ell)=\left\{k \in\{1, \ldots, n\} \mid Q_{\ell} \nabla K\left(x^{i}\right) P_{j} \nabla K\left(x^{i}\right)^{*} Q_{k} \neq 0, j \in \stackrel{\circ}{S}(i)\right\} .
$$

Then the lower bound (3.6) holds.

Proof. By the first part of (4.2), (4.1), and $\lambda_{j, \ell}^{i}=\phi_{j}^{i} \tau_{j}^{i} \chi_{\dot{S}(i)}(j)$, we have

$$
\breve{\sigma}_{\ell}^{0} \stackrel{\circ}{\tau}_{j}^{0} \geq \frac{\eta^{i} \breve{\sigma}_{\ell}^{i+1} \stackrel{\circ}{j}_{j}^{i}}{\eta^{i+1}}=\frac{\stackrel{\circ}{\pi}_{j}^{i} \phi_{j}^{i}\left(\tilde{\tau}_{j}^{i}\right)^{2}}{\psi_{\ell}^{i+1}}=\frac{\stackrel{\circ}{\pi}_{j}^{i}\left(\lambda_{j, \ell}^{i}\right)^{2}}{\psi_{\ell}^{i+1} \phi_{j}^{i}} \quad(j \in \stackrel{\circ}{S}(i)) .
$$

By the orthogonality of the projections $P_{j}$, we may insert this estimation into the second part of (4.2), obtaining (3.5); compare the proof of Theorem 3.5. The definition of $\overline{\mathcal{V}}_{j}^{i}(\ell)$ in (3.3) also reduces to that in (4.3b), while the definition of $w_{j, \ell}^{i}$ in (4.3a) is exactly that in (3.4). We finish by applying Theorem 3.5 to verify (3.6).

REMARK 4.2. The first part of (4.2) relaxes the property $\tau^{i} \sigma^{i}=\tau^{0} \sigma^{0}$ of the basic PDPS [7].

REMARK 4.3. With deterministic updates $\left(\stackrel{\circ}{\pi}_{j}^{i} \equiv 1\right)$, (4.1) couples ${ }_{\tau_{j}^{i}}^{i} \phi_{j}^{i}=\breve{\sigma}_{\ell}^{i} \psi_{\ell}^{i}$. With $\psi_{\ell}^{i} \equiv \psi_{\ell}^{0},(4.2)$ therefore becomes a block-coupled variant of the condition $\tau_{i} \sigma_{i}\|K\|^{2}<1$ from [7]. 
Algorithm 4.1 Full dual updates \#1.

Assume the problem structure (P), equivalently (S). For each iteration $i \in \mathbb{N}$, choose a sampling pattern for generating the random set of updated primal blocks $S(i) \in \mathcal{R}\left(\mathcal{O}_{i} ; \mathcal{P}\{1, \ldots, m\}\right)$ with corresponding blockwise probabilities $\dot{\pi}_{j}^{i}:=\mathbb{P}\left[j \in S(i) \mid \mathcal{O}_{i-1}\right]>0$. Also choose a rule for the iteration and block-dependent step length parameters $\dot{\tau}_{j}^{i}, \breve{\sigma}_{\ell}^{i}, \bar{\omega}^{i}>0$ from one of the Theorems 4.5, 4.4, or 4.7. Pick an initial iterate $\left(x^{0}, y^{0}\right)$, and in each iteration $i \in \mathbb{N}$, update all blocks $x_{j}^{i+1}=P_{j} x^{i+1}(j=1, \ldots, m)$ and $y_{\ell}^{i+1}=Q_{\ell} y^{i+1}(\ell=1, \ldots, n)$ of $x^{i+1}$ and $y^{i+1}$ as:

$$
\begin{aligned}
x_{j}^{i+1}: & = \begin{cases}\left(I+\dot{\tau}_{j}^{i} P_{j} \partial G_{j} P_{j}\right)^{-1}\left(x_{j}^{i}-\dot{\tau}_{j}^{i} P_{j} \nabla K\left(x^{i}\right)^{*} y^{i}\right), & j \in S(i), \\
x_{j}^{i}, & j \notin S(i),\end{cases} \\
\bar{x}_{j}^{i+1}: & = \begin{cases}x_{j}^{i+1}+\bar{\omega}^{i}\left(x_{j}^{i+1}-x_{j}^{i}\right) / \dot{\pi}_{j}^{i}, & j \in S(i), \\
x_{j}^{i}, & j \notin S(i),\end{cases} \\
y_{\ell}^{i+1}: & =\left(I+\breve{\sigma}_{\ell}^{i+1} Q_{\ell} \partial F_{\ell}^{*} Q_{\ell}\right)^{-1}\left(y_{\ell}^{i}+\breve{\sigma}_{\ell}^{i+1} Q_{\ell} K\left(\bar{x}^{i+1}\right)\right) .
\end{aligned}
$$

Finally, we also remind that for this section, (3.27) and (3.28) simplify to

$$
\begin{array}{ll}
\bar{\gamma}_{G K, j}^{i} \equiv \bar{\gamma}_{G K, j}:=\gamma_{G, j}+\gamma_{K, j}, \quad \text { and } \\
\bar{\gamma}_{F^{*}, \ell}^{i+1} \equiv \bar{\gamma}_{F^{*}, \ell}:= \begin{cases}\gamma_{F^{*}, \ell}, & Q_{\ell} P_{\mathrm{NL}}=0, \\
\gamma_{F^{*}, \ell}-(p-1) \zeta_{\ell}-\alpha_{y}, & Q_{\ell} P_{\mathrm{NL}} \neq 0 .\end{cases}
\end{array}
$$

4.1. Accelerated rates. We start with simple step length rules for $O(1 / N)$-rates for the blocks admitting second-order growth $\left(\gamma_{G, j}+\gamma_{K, j}>0\right.$ for primal blocks $j$ or $\gamma_{F^{*}, \ell}>0$ for dual blocks $\ell$ ). Throughout, for simplicity, we assume iteration-independent probabilities, $\stackrel{\circ}{\pi}_{j}^{i}=\pi_{j}^{i} \equiv \stackrel{\circ}{\pi}_{j}$ for all $i \in \mathbb{N}$.

THEOREM 4.4. Suppose that Assumptions 3.1, 3.2, and 3.4 hold with $L, L_{3} \geq 0$, $p \in[1,2], \gamma_{G, j}+\gamma_{K, j} \geq 0(j=1, \ldots, m)$, and $\bar{\gamma}_{F^{*}, \ell} \geq 0(\ell=1, \ldots, n)$, for some $\alpha_{y}, \zeta_{\ell} \geq 0$ as defined in (4.4). Let the iterates $\left\{u^{i}=\left(x^{i}, y^{i}\right)\right\}_{i \in \mathbb{N}}$ be generated by Algorithm 4.1 with iteration-independent probabilities $\stackrel{\pi}{i}_{j}^{i} \equiv \stackrel{\pi}{j}_{j}$ and step length parameters

$$
\breve{\sigma}_{\ell}^{i+1}:=\frac{\breve{\sigma}_{\ell}^{i}}{1+2 \breve{\sigma}_{\ell}^{i} \bar{\gamma}_{F^{*}, \ell}}, \quad \bar{\omega}^{i} \equiv 1, \quad \text { and } \quad \stackrel{\circ}{j}^{i+1}:=\frac{\stackrel{\circ}{\tau}_{j}^{i}}{1+2 \dot{\tau}_{j}^{i} \widetilde{\gamma}_{G, j}},
$$

with either $0 \leq \widetilde{\gamma}_{G, j}<\stackrel{\circ}{\pi}_{j}\left(\gamma_{G, j}+\gamma_{K, j}\right)$ or $\widetilde{\gamma}_{G, j}=\gamma_{G, j}+\gamma_{K, j}=0$, for each $j=1, \ldots, m$. Moreover, let the initial $\tau_{j}^{0}, \breve{\sigma}_{\ell}^{0}>0$ satisfy, for some $0<\delta<\kappa<1, \rho_{x}, \rho_{\ell} \geq 0(\ell=1, \ldots, n)$ and with $w_{j, \ell}^{i}$ as in (4.3), the bounds

$$
1-\kappa \geq\left\|\sum_{j \in \dot{S}(i)} \sqrt{\frac{w_{j, \ell}^{i} \breve{\sigma}_{\ell}^{0} \dot{\tau}_{j}^{0}}{\stackrel{\sigma}{*}_{j}}} Q_{\ell} \nabla K\left(x^{i}\right) P_{j}\right\|^{2}
$$

and

$$
\delta \geq \dot{\tau}_{j}^{0} \bar{L}+\stackrel{\circ}{\tau}_{j}^{0} \cdot \begin{cases}2\left(1-\stackrel{\circ}{\pi}_{j}\right)\left(\gamma_{G, j}+\gamma_{K, j}\right) \frac{\gamma_{G, j}+\gamma_{K, j}-\widetilde{\gamma}_{G, j}}{\pi_{j}\left(\gamma_{G, j}+\gamma_{K, j}\right)-\tilde{\gamma}_{G, j}} & \text { if } \gamma_{G, j}+\gamma_{K, j}>0 \\ 0 & \text { if } \gamma_{G, j}+\gamma_{K, j}=0\end{cases}
$$


with

(4.6c) $\bar{L}:=L_{3}+L\left(\max _{j=1, \ldots, m}\left(\frac{1}{\pi_{j}}+1\right)^{2} \sum_{\ell=1}^{n} \rho_{\ell}+\frac{n L}{2 \alpha_{y}} \rho_{x}^{2}\right) \quad(i \in \mathbb{N} ; j=1, \ldots, m)$.

Assume for $A:=\sum_{j \in S(i)}\left(\stackrel{\circ}{\pi}_{j}\right)^{-1} P_{j}$ that

$$
\begin{aligned}
\mathbb{E}_{i-1}\left[\theta_{A}\right] & \geq p^{-p} \sum_{\ell=1}^{n} \zeta_{\ell}^{1-p} \rho_{\ell}^{2-p} \quad \text { and } \\
1 & =\mathbb{P}\left[\left\|x^{i+1}-\widehat{x}\right\| \leq \rho_{x},\left\|Q_{\ell}\left(y^{i+1}-\widehat{y}\right)\right\|_{P_{\mathrm{NL}}} \leq \rho_{\ell},(\ell=1, \ldots, n) \mid \mathcal{O}_{i-1}\right]
\end{aligned}
$$

Then $\mathbb{E}\left[\left\|P_{j}\left(x^{N}-\widehat{x}\right)\right\|^{2}\right] \rightarrow 0$ at the rate $O(1 / N)$ for all $j$ such that $\widetilde{\gamma}_{G, j}>0$ and $\mathbb{E}\left[\left\|Q_{\ell}\left(y^{N}-\widehat{y}\right)\right\|^{2}\right] \rightarrow 0$ at the rate $O(1 / N)$ for all $\ell$ such that $\bar{\gamma}_{F^{*}, \ell}>0$.

Proof. We use Theorem 3.11 whose conditions we need to verify. We have already verified the nesting condition (2.9) for $\stackrel{\circ}{V}(i+1)=\emptyset, V(i+1)=\{1, \ldots, n\}$, and $\stackrel{\circ}{S}(i)=S(i)$ in Algorithm 4.1. The coupling condition (3.24), we have reduced to (4.1), which we now verify. For some $\eta^{0}>0$ we set $\eta^{i} \equiv \eta^{0}, \phi_{j}^{0}:=\eta^{0}\left(\stackrel{\circ}{\pi}_{j} \stackrel{\circ}{j}_{j}^{0}\right)^{-1}$, and $\psi_{\ell}^{0}:=\eta^{0} / \breve{\sigma}_{\ell}^{0}$. Then we update

$$
\phi_{j}^{i+1}=\left(1+2 \stackrel{\circ}{\tau}_{j}^{i} \widetilde{\gamma}_{G, j}\right) \phi_{j}^{i}, \quad \psi_{\ell}^{i+2}=\left(1+2 \breve{\sigma}_{\ell}^{i+1} \bar{\gamma}_{F^{*}, \ell}\right) \psi_{\ell}^{i+1}
$$

By (4.5), consequently, $\breve{\sigma}_{\ell}^{i+1} \psi_{\ell}^{i+1}=\eta^{i+1}=\stackrel{\circ}{\pi}_{j} \phi_{j}^{i+1} \dot{\tau}_{j}^{i+1}$ for all $\ell$ and $j$. Consequently, (4.1) holds. Clearly, so does (3.14) due to the deterministic step length and testing parameter updates. The conditions (3.25) follow from (4.7) given that $\theta_{\Phi_{i} T_{i}}=\eta^{i} \theta_{A}=\eta^{i+1} \theta_{A}=\breve{\sigma}_{\ell}^{i+1} \psi_{\ell}^{i+1} \theta_{A}$.

The step length parameters $\stackrel{i}{\tau}_{j}^{i}$ and $\breve{\sigma}_{\ell}^{i+1}$ are non-increasing in $i$ by the defining identities (4.5). Also using (4.6a), we thus verify (4.2). Now Theorem 4.1 verifies (3.6).

We still need to verify Theorem 3.11 (i) and (ii). Regarding the latter, the inequality $\psi_{\ell}^{i+2} \leq\left(1+2 \breve{\sigma}_{\ell}^{i+1} \bar{\gamma}_{F^{*}, \ell}^{i+1}\right) \psi_{\ell}^{i+1}$ holds trivially as long as $\bar{\gamma}_{F^{*}, \ell}^{i+1} \geq 0$, which follows from the assumptions on $\gamma_{F^{*}, \ell}$. Therefore, Theorem 3.11 (ii) option (a) holds. Regarding Theorem 3.11 (i), we first of all observe that (3.23) reduces to $c_{*}^{i}=n L^{2} \eta^{i+1} \rho_{x}^{2} /\left(2 \alpha_{y}\right)$. Moreover, in Algorithm 4.1 we took $\omega_{j}^{i}:=\bar{\omega}^{i} / \stackrel{\circ}{\pi}_{j}=1 / \stackrel{\circ}{\pi}_{j}$ by (4.5). Consequently, (3.26) becomes

$$
\begin{aligned}
L_{j}^{i} & :=L_{3}+\left(L \max _{j \in S(i)}\left(\omega_{j}^{i}+1\right)^{2} \sum_{\ell=1}^{m} \psi_{\ell}^{i+1} \breve{\sigma}_{\ell}^{i+1} \rho_{\ell}+\frac{n L^{2} \eta^{i+1} \rho_{x}^{2}}{2 \alpha_{y}}\right) \frac{1}{\phi_{j}^{i} \tilde{\tau}_{j}^{i}} \\
& =L_{3}+L \stackrel{\circ}{\pi}_{j}\left(\max _{j \in S(i)}\left(1 / \stackrel{\circ}{\pi}_{j}+1\right)^{2} \sum_{\ell=1}^{n} \rho_{\ell}+\frac{n L}{2 \alpha_{y}} \rho_{x}^{2}\right) \frac{\eta^{i+1}}{\eta^{i}} \leq \bar{L}
\end{aligned}
$$

We now consider two cases for satisfying Theorem 3.11 (i) option (a) or (b):

(A) If $\gamma_{G, j}+\gamma_{K, j}=0$, then $\widetilde{\gamma}_{G, j}=0$ and $\phi_{j}^{i+1}=\phi_{j}^{i}$ by (4.8), so option (a) holds.

(B) If $\gamma_{G, j}+\gamma_{K, j}>0$, then (4.6b), (4.9), and $\stackrel{\circ}{j}_{j}^{i} \leq \stackrel{\circ}{\tau}_{j}^{0}$ show (3.40a), hence (b) holds.

We can now apply Theorem 3.11 to obtain (3.29). From (4.8) we have

$$
\begin{aligned}
& \phi_{j}^{i+1}=\phi_{j}^{i}+2 \widetilde{\gamma}_{G, j} \eta^{i} / \stackrel{\pi}{\pi}_{j}=\phi_{j}^{i}+2 \widetilde{\gamma}_{G, j} \eta^{1} / \stackrel{\circ}{\pi}_{j}=\ldots=\phi_{j}^{1}+2 i \widetilde{\gamma}_{G, j} \eta^{1} / \AA_{j} \quad \text { and } \\
& \psi_{\ell}^{i+2}=\psi_{\ell}^{i+1}+2 \bar{\gamma}_{F^{*}, \ell} \eta^{i+1}=\psi_{\ell}^{i+1}+2 \bar{\gamma}_{F^{*}, \ell} \eta^{1}=\ldots=\psi_{\ell}^{1}+2(i+1) \bar{\gamma}_{F^{*}, \ell} \eta^{1} .
\end{aligned}
$$

Therefore, for any $j$ such that $\widetilde{\gamma}_{G, j}>0$ and $\ell$ such that $\bar{\gamma}_{F^{*}, \ell}>0, \phi_{j}^{N}$ and $\psi_{\ell}^{N+1}$ grow as $\Omega(N)$. This together with (3.29) yields the claim.

We can improve the convergence to $O\left(1 / N^{2}\right)$ in the primal variable if all the primal blocks exhibit second-order growth. This is achieved by making the dual step lengths grow as in the basic single-block convex case of [7]. 
THEOREM 4.5. Suppose that Assumption 3.1, 3.2, and 3.4 hold with $L, L_{3} \geq 0$, $p \in[1,2], \gamma_{G, j}+\gamma_{K, j}>0(j=1, \ldots, m)$, and $\bar{\gamma}_{F^{*}, \ell} \geq 0(\ell=1, \ldots, n)$, for some $\alpha_{y}, \zeta_{\ell} \geq 0$ as defined in (4.4). Let the iterates $\left\{u^{i}=\left(x^{i}, y^{i}\right)\right\}_{i \in \mathbb{N}}$ be generated by Algorithm 4.1 with iteration-independent probabilities $\stackrel{\pi}{i}_{j}^{i} \equiv \stackrel{\pi}{j}_{j}$ and step length parameters

$$
\breve{\sigma}_{\ell}^{i+1}:=\frac{\breve{\sigma}_{\ell}^{i}}{\bar{\omega}^{i}}, \quad \stackrel{\tau}{\tau}_{j}^{i+1}:=\frac{1}{1+2 \tilde{\tau}_{j}^{i} \widetilde{\gamma}_{G, j}} \frac{\stackrel{\circ}{\tau}_{j}^{i}}{\bar{\omega}^{i}}, \quad \text { and } \quad \bar{\omega}^{i}:=\max _{j=1, \ldots, m} \frac{1}{\sqrt{1+2 \tau_{j}^{i} \widetilde{\gamma}_{G, j}}}
$$

with $0<\widetilde{\gamma}_{G, j}<\stackrel{\pi}{\pi}_{j}\left(\gamma_{G, j}+\gamma_{K, j}\right)$. Moreover, let the initial $\stackrel{\tau}{j}_{j}^{0}, \breve{\sigma}_{\ell}^{0}>0$ satisfy, for some $0<\delta \leq \kappa<1, \rho_{x}, \rho_{\ell} \geq 0(\ell=1, \ldots, n)$ and with $w_{j, \ell}^{i}$ as in (4.3), the bounds

$$
\begin{aligned}
& 1-\kappa \geq\left\|\sum_{j \in \grave{S}(i)} \sqrt{\frac{w_{j, \ell}^{i} \breve{\sigma}_{\ell}^{0} \dot{\tau}_{j}^{0}}{\tilde{\pi}_{j}}} Q_{\ell} \nabla K\left(x^{i}\right) P_{j}\right\|^{2} \quad(i \in \mathbb{N}) \text { and } \\
& \delta \geq \stackrel{\circ}{\tau}_{j}^{0}\left(\bar{L}+2\left(1-\stackrel{\circ}{\pi}_{j}\right)\left(\gamma_{G, j}+\gamma_{K, j}\right) \frac{\gamma_{G, j}+\gamma_{K, j}-\widetilde{\gamma}_{G, j}}{\pi_{j}\left(\gamma_{G, j}+\gamma_{K, j}\right)-\widetilde{\gamma}_{G, j}}\right) \text { with } \\
& \bar{L}:=L_{3}+\frac{L}{\bar{\omega}^{0}}\left(\max _{j=1, \ldots, m}\left(\frac{1}{\pi_{j}}+1\right)^{2} \sum_{\ell=1}^{n} \rho_{\ell}+\frac{n L}{2 \alpha_{y}} \rho_{x}^{2}\right) .
\end{aligned}
$$

Assume for $A:=\sum_{j \in S(i)}\left(\stackrel{\circ}{\pi}_{j}\right)^{-1} P_{j}$ that

$$
\begin{aligned}
& \mathbb{E}_{i-1}\left[\theta_{A}\right] \geq p^{-p} \sum_{\ell=1}^{n} \zeta_{\ell}^{1-p} \rho_{\ell}^{2-p} / \bar{\omega}^{0} \quad \text { and } \\
& 1=\mathbb{P}\left[\left\|x^{i+1}-\widehat{x}\right\| \leq \rho_{x},\left\|Q_{\ell}\left(y^{i+1}-\widehat{y}\right)\right\|_{P_{\mathrm{NL}}} \leq \rho_{\ell},(\ell=1, \ldots, n) \mid \mathcal{O}_{i-1}\right] .
\end{aligned}
$$

Then $\mathbb{E}\left[\left\|P_{j}\left(x^{N}-\widehat{x}\right)\right\|^{2}\right] \rightarrow 0$ at the rate $O\left(1 / N^{2}\right)$ for all $j$.

Proof. We use Theorem 3.11 whose conditions we need to verify. We have already verified the nesting conditions (2.9) for the choices ${ }^{\circ}(i+1)=\emptyset, V(i+1)=\{1, \ldots, n\}$, and $\dot{S}(i)=S(i)$ in Algorithm 4.1. The coupling condition (3.24) we have reduced to (4.1). To verify (4.1), we initialise $\phi_{j}^{0}:=\eta^{0}\left(\stackrel{\circ}{\pi}_{j}^{0} \stackrel{\tau}{\gamma}_{j}^{0}\right)^{-1}$ and $\psi_{\ell}^{0}:=\eta^{0} / \breve{\sigma}_{\ell}^{0}$ for some $\eta^{0}>0$ and update

$$
\phi_{j}^{i+1}:=\left(1+2 \dot{\tau}_{j}^{i} \widetilde{\gamma}_{G, j}\right) \phi_{j}^{i}, \quad \psi_{\ell}^{i+1}:=\psi_{\ell}^{i}, \quad \text { and } \quad \eta^{i+1}:=\eta^{i} / \bar{\omega}^{i} .
$$

Then from (4.10), $\psi_{\ell}^{i+1} \breve{\sigma}_{\ell}^{i+1}=\psi_{\ell}^{i} \breve{\sigma}_{\ell}^{i} / \bar{\omega}^{i}$ and $\phi_{j}^{i+1}{\tau_{j}^{i+1}}^{i+1}=\phi_{j}^{i} \stackrel{\tau}{j}_{j}^{i} / \bar{\omega}^{i}$. Therefore, (4.1) holds by induction. Clearly, also (3.14) holds due to the step length and testing parameters being updated deterministically. Conditions (3.25) follow from (4.12) and (4.1) given that $\tau_{j}^{i}$ decreases, so $\bar{\omega}^{i} \geq \bar{\omega}_{0}$ and $\theta_{\Phi_{i} T_{i}}=\eta^{i} \theta_{A}=\eta^{i+1} \bar{\omega}^{i} \theta_{A}$. yield

We now verify (3.6). By (4.10) and (4.13), we get $\phi_{j}^{i+1}\left(\dot{\tau}_{j}^{i+1}\right)^{2} \leq \phi_{j}^{i}\left(\left(_{j}^{i}\right)^{2}\right.$. This and (4.1)

$$
\bar{\omega}^{i} \breve{\sigma}_{\ell}^{i+1} \stackrel{\sim}{\tau}_{j}^{i}=\frac{\eta^{i} \tau_{j}^{i}}{\psi_{\ell}^{i+1}}=\frac{\phi_{j}^{i}\left(\dot{\tau}_{j}^{i}\right)^{2}}{\psi_{\ell}^{i+1} \stackrel{\pi}{\pi}_{j}} \leq \frac{\phi_{j}^{0}\left(\stackrel{\circ}{\tau}_{j}^{0}\right)^{2}}{\psi_{\ell}^{i+1} \stackrel{\circ}{\pi}_{j}}=\frac{\eta^{0} \stackrel{\tau}{j}_{j}^{0}}{\psi_{\ell}^{0}}=\breve{\sigma}_{\ell}^{0} \stackrel{\tau}{\tau}_{j}^{0} .
$$

Combining this estimate with (4.11a), we verify (4.2). Thus, Theorem 4.1 establishes (3.6).

We still need to verify Theorem 3.11 (i) and (ii). Regarding the dual test, the bound $\psi_{\ell}^{i+2}=\psi_{\ell}^{i+1} \leq\left(1+2 \breve{\sigma}_{\ell}^{i+1} \bar{\gamma}_{F^{*}, \ell}^{i+1}\right) \psi_{\ell}^{i+1}$ holds trivially as long as $\bar{\gamma}_{F^{*}, \ell}^{i+1} \geq 0$, which follows from the assumptions on $\gamma_{F^{*}, \ell}$. Therefore, Theorem 3.11 (ii) option (a) holds. As far as Theorem 3.11 (i) is concerned, we observe that (3.23) reduces to $c_{*}^{i}=n L^{2} \eta^{i+1} \rho_{x}^{2} /\left(2 \alpha_{y}\right)$. Consequently, (3.26) becomes

$$
L_{j}^{i}:=L_{3}+L \stackrel{\circ}{\pi}_{j}\left(\max _{j \in S(i)}\left(\omega_{j}^{i}+1\right)^{2} \sum_{\ell=1}^{n} \rho_{\ell}+\frac{n L}{2 \alpha_{y}} \rho_{x}^{2}\right) \eta^{i+1} / \eta^{i} \leq \bar{L}
$$




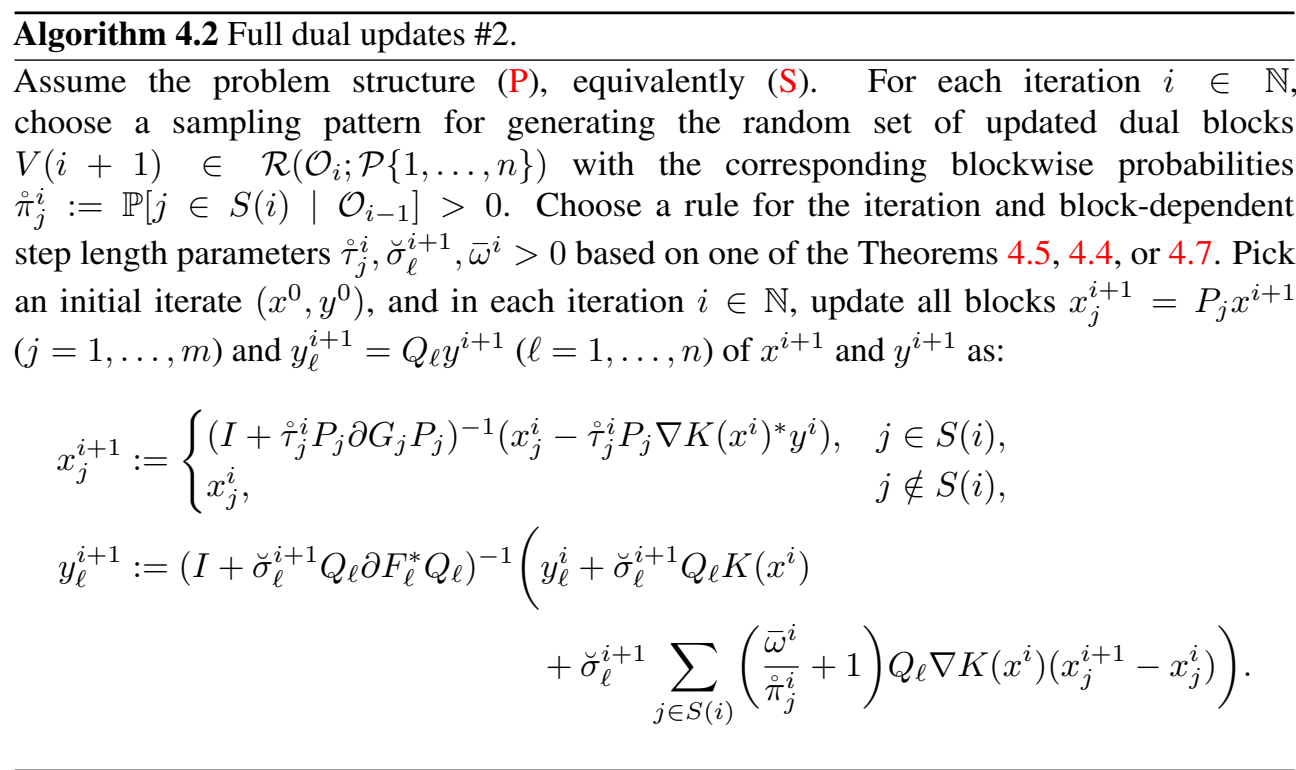

thanks to $\omega_{j}^{i}:=\bar{\omega}^{i} / \AA_{j} \leq 1 / \stackrel{\circ}{\pi}_{j}$ and $\bar{\omega}^{i} \geq \bar{\omega}^{0}$. Also, with $\widetilde{\gamma}_{G, j}^{i}<\left(\stackrel{\circ}{\pi}_{j} \gamma_{G, j}+\gamma_{K, j}\right),(4.11 \mathrm{~b})$, (4.14), and $\tau_{j}^{i} \leq \dot{\tau}_{j}^{0}$ show (3.40a), hence, (3.30). Therefore, Theorem 3.11(i) option (b) holds for every $j=1, \ldots, m$.

We can thus apply Theorem 3.11 to obtain (3.29). Multiplying the $\tau$-update of (4.10) by $2 \widetilde{\gamma}_{G, j}$, plugging in $\bar{\omega}^{i}$, and taking the inverse, we have

$$
\begin{aligned}
\left(2 \tau_{j}^{i+1} \widetilde{\gamma}_{G, j}\right)^{-1} & =\frac{1+2 \dot{\tau}_{j}^{i} \widetilde{\gamma}_{G, j}}{2 \tilde{\tau}_{j}^{i} \widetilde{\gamma}_{G, j} \sqrt{1+\min _{j=1, \ldots, m}\left(2 \dot{\tau}_{j}^{i} \widetilde{\gamma}_{G, j}\right)}} \\
& =\frac{1+\left(2 \tau_{j}^{i} \widetilde{\gamma}_{G, j}\right)^{-1}}{\sqrt{1+\left(\max _{j=1, \ldots, m}\left(2 \tau_{j}^{i} \widetilde{\gamma}_{G, j}\right)^{-1}\right)^{-1}}}
\end{aligned}
$$

We now apply Theorem B.1 with $z_{j}^{i}=\left(2 \dot{\tau}_{j}^{i} \widetilde{\gamma}_{G, j}\right)^{-1}$ to get

$$
\max _{j=1, \ldots, m}\left(2 \AA_{j}^{N} \widetilde{\gamma}_{G, j}\right)^{-1} \leq \bar{z}_{0}+N / 2
$$

with $\bar{z}_{0}>0$. Then from (4.13), we have

$$
\begin{aligned}
\phi_{j}^{N+1} & \geq\left(1+\min _{j=1, \ldots, m}\left(2 \stackrel{\circ}{i}_{j}^{i} \widetilde{\gamma}_{G, j}\right)\right) \phi_{j}^{N} \geq\left(1+\frac{1}{\bar{z}_{0}+N / 2}\right) \phi_{j}^{N}=\frac{2 \bar{z}_{0}+N+2}{2 \bar{z}_{0}+N} \phi_{j}^{N} \\
& =\frac{2 \bar{z}_{0}+N+2}{2 \bar{z}_{0}+N} \frac{2 \bar{z}_{0}+N+1}{2 \bar{z}_{0}+N-1} \phi_{j}^{N-1}=\ldots=\frac{\left(2 \bar{z}_{0}+N+2\right)\left(2 \bar{z}_{0}+N+1\right)}{2 \bar{z}_{0}\left(2 \bar{z}_{0}+1\right)} \phi_{j}^{0} .
\end{aligned}
$$

Therefore, $\phi_{j}^{N}$ grows as $\Omega\left(N^{2}\right)$, so we obtain the claimed convergence rates from (3.29).

In Algorithm 4.1, we chose $\omega_{j}^{i}$ to eliminate the $\nabla K\left(x^{i}\right)$-term from the dual step. Selecting $\omega_{j}^{i}=-1$ keeps this term but eliminates the necessity to have a finite $\rho_{\ell}$ as long as $p=2$, as (3.26) and (3.25b) will no longer depend on it. This yields Algorithm 4.2 and the following corollary: 


\section{ETNA}

Kent State University and

Johann Radon Institute (RICAM)

COROLlary 4.6. Theorems 4.4 and 4.5 apply to Algorithm 4.2 if Assumption 3.2 holds with $p=2$, and instead of (4.6c), (4.11c), (4.7b), and (4.12b), we assume

$$
\bar{L}:=L_{3}+n L^{2} \rho_{x}^{2} /\left(2 \alpha_{y}\right) \quad \text { and } \quad \mathbb{P}\left[\left\|x^{i+1}-\widehat{x}\right\| \leq \rho_{x} \mid \mathcal{O}_{i-1}\right]=1 .
$$

Proof. The proof remains exactly the same as those of Theorems 4.4 and 4.5 . Inserting $\omega_{j}^{i}=-1$, then (4.9) and (4.14) as well as (4.7a) and (4.12a) loose their dependency on $\rho_{\ell}$. Hence, $\rho_{\ell}$ can be taken infinitely large.

4.2. Linear convergence. If all the primal and dual blocks exhibit second-order growth, i.e., $\bar{\gamma}_{F^{*}, \ell}>0$ and $\gamma_{G, j}+\gamma_{K, j}>0$, then we obtain linear convergence:

THEOREM 4.7. Suppose that Assumptions 3.1, 3.2, and 3.4 hold with $L, L_{3} \geq 0$, $p \in[1,2], \gamma_{G, j}+\gamma_{K, j}>0(j=1, \ldots, m)$, and $\bar{\gamma}_{F^{*}, \ell}>0(\ell=1, \ldots, n)$, for some $\alpha_{y}, \zeta_{\ell} \geq 0$ as defined in (4.4). Let the iterates $\left\{u^{i}=\left(x^{i}, y^{i}\right)\right\}_{i \in \mathbb{N}}$ be generated by Algorithm 4.1 with iteration-independent probabilities $\stackrel{\circ}{\pi}_{j}^{i} \equiv \stackrel{\circ}{\pi}_{j}$ and step length parameters

$$
\begin{aligned}
\dot{\tau}_{j}^{i+1} & :=\frac{\stackrel{\circ}{\tau}_{j}^{i}}{\left(1+2 \dot{\tau}_{j}^{i} \widetilde{\gamma}_{G, j}\right) \bar{\omega}}, \quad \breve{\sigma}_{\ell}^{i+1}:=\frac{\breve{\sigma}_{\ell}^{i}}{\left(1+2 \breve{\sigma}_{\ell}^{i} \bar{\gamma}_{F^{*}, \ell}\right) \bar{\omega}}, \quad \text { and } \\
\bar{\omega}^{i} & \equiv \bar{\omega}:=\max \left\{\max _{j=1, \ldots, m} \frac{1}{1+2 \dot{\tau}_{j}^{0} \widetilde{\gamma}_{G, j}}, \max _{\ell=1, \ldots, n} \frac{1}{1+2 \breve{\sigma}_{\ell}^{0} \bar{\gamma}_{F^{*}, \ell}}\right\}
\end{aligned}
$$

with $0<\widetilde{\gamma}_{G, j}<\stackrel{\circ}{\pi}_{j}\left(\gamma_{G, j}+\gamma_{K, j}\right)$. Moreover, let the initial $\stackrel{\circ}{j}_{j}^{0}, \breve{\sigma}_{\ell}^{0}>0$ satisfy, for some $0<\delta<\kappa<1, \rho_{x}, \rho_{\ell} \geq 0$, $(\ell=1, \ldots, n)$ and with $w_{j, \ell}^{i}$ as in (4.3), the bounds

$$
1-\kappa \geq\left\|\sum_{j \in \dot{S}(i)} \sqrt{\frac{w_{j, \ell}^{i} \breve{\sigma}_{\ell}^{0} \tau_{j}^{0}}{\check{\pi}_{j}}} Q_{\ell} \nabla K\left(x^{i}\right) P_{j}\right\|^{2} \quad \text { and }
$$

$$
\begin{aligned}
& \delta \geq \stackrel{\circ}{j}_{j}^{0}\left(\bar{L}+2\left(1-\stackrel{\circ}{\pi}_{j}\right)\left(\gamma_{G, j}+\gamma_{K, j}\right) \frac{\gamma_{G, j}+\gamma_{K, j}-\widetilde{\gamma}_{G, j}}{\pi_{j}\left(\gamma_{G, j}+\gamma_{K, j}\right)-\widetilde{\gamma}_{G, j}}\right) \quad \text { with } \\
& \bar{L}:=L_{3}+\frac{L}{\bar{\omega}}\left(\max _{j=1, \ldots, m}\left(\frac{\bar{\omega}}{\tilde{\pi}_{j}}+1\right)^{2} \sum_{\ell=1}^{n} \rho_{\ell}+\frac{n L}{2 \alpha_{y}} \rho_{x}^{2}\right) \quad(i \in \mathbb{N}, j \in S(i)) .
\end{aligned}
$$

Further assume for $A:=\sum_{j \in S(i)}\left(\stackrel{\circ}{j}_{j}\right)^{-1} P_{j}$ that

$$
\begin{aligned}
& \mathbb{E}_{i-1}\left[\theta_{A}\right] \geq p^{-p} \sum_{\ell=1}^{n} \zeta_{\ell}^{1-p} \rho_{\ell}^{2-p} / \bar{\omega} \quad \text { and } \\
& 1=\mathbb{P}\left[\left\|x^{i+1}-\widehat{x}\right\| \leq \rho_{x},\left\|Q_{\ell}\left(y^{i+1}-\widehat{y}\right)\right\|_{P_{\mathrm{NL}}} \leq \rho_{\ell}, \quad(\ell=1, \ldots, n) \mid \mathcal{O}_{i-1}\right] .
\end{aligned}
$$

Then $\mathbb{E}\left[\left\|P_{j}\left(x^{N}-\widehat{x}\right)\right\|^{2}\right]$ and $\mathbb{E}\left[\left\|Q_{\ell}\left(y^{N}-\widehat{y}\right)\right\|^{2}\right]$ converge to zero at the linear rate $O\left((1 / \bar{\omega})^{N}\right)$ for all $j \in\{1, \ldots, m\}$ and $\ell \in\{1, \ldots, n\}$.

Proof. We use Theorem 3.11 whose conditions we need to verify. We have already verified the nesting condition (2.9) for the choices ${ }^{V}(i+1)=\emptyset, V(i+1)=\{1, \ldots, n\}$, and $\stackrel{S}{S}(i)=S(i)$ in Algorithm 4.1. The coupling condition (3.24) we have reduced to (4.1). To verify (4.1), we initialise $\phi_{j}^{0}:=\eta^{0}\left(\stackrel{\pi}{\pi}_{j}^{0} \tilde{\tau}_{j}^{0}\right)^{-1}$ and $\psi_{\ell}^{0}:=\eta^{0} / \breve{\sigma}_{\ell}^{0}$ for some $\eta^{0}>0$ and update

$$
\phi_{j}^{i+1}:=\left(1+2 \dot{\tau}_{j}^{i} \widetilde{\gamma}_{G, j}\right) \phi_{j}^{i}, \quad \psi_{\ell}^{i+1}:=\left(1+2 \breve{\sigma}_{\ell}^{i} \bar{\gamma}_{F^{*}, \ell}\right) \psi_{\ell}^{i}, \quad \text { and } \quad \eta^{i+1}:=\eta^{i} / \bar{\omega} .
$$

Then from (4.15), $\psi_{\ell}^{i+1} \breve{\sigma}_{\ell}^{i+1}=\psi_{\ell}^{i} \breve{\sigma}_{\ell}^{i} / \bar{\omega}$ and $\phi_{j}^{i+1} \overbrace{j}^{i+1}=\phi_{j}^{i} \overbrace{j}^{i} / \bar{\omega}$. Therefore, (4.1) holds by induction. Clearly, also (3.14) holds as the step length and testing parameters are updated deterministically. The conditions (3.25) follow from (4.17) given that $\theta_{\Phi_{i} T_{i}}=\eta^{i} \theta_{A}=\bar{\omega} \eta^{i+1} \theta_{A}$. 
We now prove (3.6). We start by proving by induction that

$$
\bar{\omega}=\max \left\{\max _{j=1, \ldots, m} \frac{1}{1+2 \tau_{j}^{i} \widetilde{\gamma}_{G, j}}, \max _{\ell=1, \ldots, n} \frac{1}{1+2 \breve{\sigma}_{\ell}^{i} \bar{\gamma}_{F^{*}, \ell}}\right\}
$$

in other words, $\bar{\omega}^{-1}=1+\min \left\{\min _{j=1, \ldots, m} 2 \dot{\tau}_{j}^{i} \widetilde{\gamma}_{G, j}, \min _{\ell=1, \ldots, n} 2 \breve{\sigma}_{\ell}^{i} \bar{\gamma}_{F^{*}, \ell}\right\}$. The inductive base for $i=0$ is clear from (4.15b). Using (4.15a), we obtain

$$
\begin{aligned}
\min \left\{\min _{j=1, \ldots, m} 2 \dot{\tau}_{j}^{i+1} \widetilde{\gamma}_{G, j}, \min _{\ell=1, \ldots, n} 2 \breve{\sigma}_{\ell}^{i+1} \bar{\gamma}_{F^{*}, \ell}\right\} \\
=\frac{1}{\bar{\omega}} \min \left\{\min _{j=1, \ldots, m} \frac{1}{1+\left(2 \tilde{\tau}_{j}^{i} \widetilde{\gamma}_{G, j}\right)^{-1}}, \min _{\ell=1, \ldots, n} \frac{1}{1+\left(2 \breve{\sigma}_{\ell}^{i} \bar{\gamma}_{F^{*}, \ell}\right)^{-1}}\right\} \\
=\frac{1}{\bar{\omega}} \frac{1}{1+\min ^{-1}\left\{\min _{j=1, \ldots, m} 2 \dot{\tau}_{j}^{i} \widetilde{\gamma}_{G, j}, \min _{\ell=1, \ldots, n} 2 \breve{\sigma}_{\ell}^{i} \bar{\gamma}_{F^{*}, \ell}\right\}} \\
=\min \left\{\min _{j=1, \ldots, m} 2 \dot{\tau}_{j}^{i} \widetilde{\gamma}_{G, j}, \min _{\ell=1, \ldots, n} 2 \breve{\sigma}_{\ell}^{i} \bar{\gamma}_{F^{*}, \ell}\right\} .
\end{aligned}
$$

This establishes the inductive step, hence (4.19). By (4.19) and (4.15a), $\stackrel{\tau}{j}^{i+1}$ and $\breve{\sigma}_{\ell}^{i+1}$ are non-increasing in $i$. Also using (4.16a), this verifies (4.2). Thus, Theorem 4.1 verifies (3.6).

We need to verify Theorem 3.11 (i) and (ii). Option (a) of the latter is trivially satisfied for every $\ell=1, \ldots, n$ based on (4.18). Regarding Theorem 3.11 (i), we first of all observe that (3.23) reduces to $c_{*}^{i}=n L^{2} \eta^{i+1} \rho_{x}^{2} /\left(2 \alpha_{y}\right)$. Consequently, (3.26) becomes

$$
L_{j}^{i}:=L_{3}+L \stackrel{\circ}{\pi}_{j}\left(\max _{j \in S(i)}\left(\omega_{j}^{i}+1\right)^{2} \sum_{\ell=1}^{n} \rho_{\ell}+\frac{n L}{2 \alpha_{y}} \rho_{x}^{2}\right) \eta^{i+1} / \eta^{i} \leq \bar{L}
$$

for $\omega_{j}^{i}:=\bar{\omega}^{i} / \stackrel{\circ}{\pi}_{j}$ as in Algorithm 4.1. And with $\widetilde{\gamma}_{G, j}<\stackrel{\circ}{\pi}_{j}\left(\gamma_{G, j}+\gamma_{K, j}\right)$, the inequalities (4.16b), (4.20), and $\dot{\tau}_{j}^{i+1} \leq \dot{\tau}_{j}^{0}$ show (3.40a). Therefore, Theorem 3.11(i) option (b) holds for every $j=1, \ldots, m$.

We can now apply Theorem 3.11 to obtain (3.29). By (4.18) and (4.19) we have

$$
\begin{aligned}
& \phi_{j}^{N+1}=\left(1+2 \dot{\tau}_{j}^{N} \widetilde{\gamma}_{G, j}\right) \phi_{j}^{N} \geq \phi_{j}^{N} / \bar{\omega} \geq \ldots \geq \phi_{j}^{0} / \bar{\omega}^{N+1} \text { and } \\
& \psi_{\ell}^{N+1}=\left(1+2 \breve{\sigma}_{\ell}^{N} \bar{\gamma}_{F^{*}, \ell}\right) \psi_{\ell}^{N} \geq \psi_{\ell}^{N} / \bar{\omega} \geq \ldots \geq \psi_{\ell}^{0} / \bar{\omega}^{N+1} .
\end{aligned}
$$

Applying these estimates in (3.29) establishes the claimed linear convergence rates.

Similarly to Algorithm 4.2, we could, in the derivation of Algorithm 4.1, set $\omega_{j}^{i}=-1$ to remove any dependencies on $\rho_{\ell}$ from (4.16c) and (4.17a). This yields Algorithm 4.2 and the following result:

COROLlary 4.8. Theorem 4.7 applies to Algorithm 4.2 if Assumption 3.2 holds with $p=2$ and (4.16c) and (4.17b) are replaced with

$$
\bar{L} \geq L_{3}+n L^{2} \rho_{x}^{2} /\left(2 \alpha_{y} \bar{\omega}\right) \quad \text { and } \quad \mathbb{P}\left[\left\|x^{i+1}-\widehat{x}\right\| \leq \rho_{x} \mid \mathcal{O}_{i-1}\right]=1 .
$$

Proof. The proof remains exactly the same as in Theorem 4.7 given all $\omega_{j}^{i}=-1$ in (4.20) and (4.17a) no longer depend on $\rho_{\ell}$, hence $\rho_{\ell}$ can be taken infinitely large.

REMARK 4.9 (Stochastic block-coordinate forward-backward splitting). Let $F(z):=z$ for $z \in \mathbb{R}$ and $K \in C^{1}(X)$. Then $F^{*}(y)=\delta_{\{1\}}(y)$. Taking $n=1$ and $Q_{1}=I$ results in $\left(I+\breve{\sigma}_{1}^{i+1} Q_{1} \partial F^{*} Q_{1}\right)^{-1} \equiv 1$. Consequently $y^{i} \equiv 1$ in all iterations, so that the updates of Algorithms 4.1 and 4.2 reduce to

$$
x_{j}^{i+1}:= \begin{cases}\left(I+\dot{\tau}_{j}^{i} P_{j} \partial G_{j} P_{j}\right)^{-1}\left(x_{j}^{i}-\dot{\tau}_{j}^{i} P_{j} \nabla K(x)\right), & j \in S(i), \\ x_{j}^{i}, & j \notin S(i),\end{cases}
$$


In the step length conditions of Theorems 4.4, 4.5, and 4.7, we can moreover take $\rho_{1}=0$ and let $\gamma_{F^{*}, 1} \nearrow \infty$, consequently $a_{y} \nearrow \infty$. In particular, in all the theorems, $\bar{L}=L_{3}$, so that when $\stackrel{\pi}{\pi}_{j}=1$, the upper bounds for the primal step lengths reduce to $\delta \geq \overbrace{j}^{0} L_{3}$ for some $\delta \in(0,1)$ similarly to the standard condition in forward-backward splitting-type methods. Moreover, by (A.1), $\gamma_{K, 1}$ is simply a (reduced) factor of strong monotonicity of $K$ at $\widehat{x}$ as defined in Assumption 3.4. Finally, since we can take $\breve{\sigma}_{1}^{0}>0$ arbitrarily small without affecting the updates (4.21), the conditions in the theorems corresponding to (3.5) become irrelevant.

5. Methods with full primal updates. We continue with developing more specific methods and their convergence results based on the updates of (2.13) and the conditions of Theorem 3.11. We now take $\stackrel{S}{S}(i)=\emptyset, S(i)=\{1, \ldots, m\}$, and $\dot{V}(i+1)=V(i+1)$ for all iterations $i$. Then the nesting condition (2.9) of Theorem 3.11 holds, and the coupling condition (3.24) becomes

$$
\phi_{j}^{i} \breve{\tau}_{j}^{i}=\eta^{i+1}=\stackrel{\circ}{\nu}_{\ell}^{i+2} \psi_{\ell}^{i+2} \stackrel{\circ}{\sigma}_{\ell}^{i+2} .
$$

Taking $\Omega_{i}=-I$, the updates of (2.13) simplify to those of Algorithm 5.1 since for the last two terms in the primal update we have

$$
\breve{\tau}_{j}^{i} y_{\ell}^{i+1}+\frac{\psi_{\ell}^{i+1} \sigma_{\ell}^{i+1}}{\phi_{j}^{i}}\left(y_{\ell}^{i+1}-y_{\ell}^{i}\right)=\breve{\tau}_{j}^{i}\left(y_{\ell}^{i+1}+\frac{\bar{\omega}^{i}}{\nu_{\ell}^{i+1}}\left(y_{\ell}^{i+1}-y_{\ell}^{i}\right)\right) \quad \text { for } \quad \bar{\omega}^{i}:=\frac{\eta^{i}}{\eta^{i+1}} .
$$

Moreover, (2.12) reduces to $\lambda_{j, \ell}^{i}=-\sigma_{\ell}^{i+1} \psi_{\ell}^{i+1}$. We thus verify (3.6) via the following lemma:

LEMma 5.1. Suppose that $\stackrel{\circ}{S}(i)=\emptyset, S(i)=\{1, \ldots, m\}$, and $\stackrel{\circ}{V}(i+1)=V(i+1)$ for $i \in \mathbb{N}$, the coupling condition (5.1) holds, and $\bar{\omega}^{i} \leq 1$. Moreover, suppose that for all $\ell=1, \ldots, n, j=1, \ldots, m$,

$$
\stackrel{\circ}{\sigma}_{\ell}^{i+1} \breve{\tau}_{j}^{i} \leq \stackrel{\sigma}{\sigma}_{\ell}^{1} \breve{\tau}_{j}^{0}, \quad \text { and } \quad 1-\kappa \geq\left\|\sum_{j=1}^{m} \sqrt{\frac{w_{j, \ell}^{i} \stackrel{\circ}{\ell}_{\ell}^{1} \breve{\tau}_{j}^{0}}{\nu_{\ell}^{i+1}}} Q_{\ell} \nabla K\left(x^{i}\right) P_{j}\right\|^{2}
$$

for some $0 \leq \kappa \leq 1$ and $w_{j, \ell, k}=1 / w_{j, k, \ell}>0$ such that

$$
w_{j, \ell}^{i}:=\chi_{\mathcal{V}_{j}^{i}}(\ell) \sum_{k \in \overline{\mathcal{V}}_{j}^{i}(\ell)} w_{j, \ell, k}
$$

with

$$
\overline{\mathcal{V}}_{j}^{i}(\ell)=\left\{k \in\{1, \ldots, n\} \mid Q_{\ell} \nabla K\left(x^{i}\right) P_{j} \nabla K\left(x^{i}\right)^{*} Q_{k} \neq 0, \ell \in \stackrel{\circ}{V}(i+1)\right\} .
$$

Then the lower bound (3.6) holds.

Proof. By the first part of (5.2), (5.1), and $\lambda_{j, \ell}^{i}=-\sigma_{\ell}^{i+1} \psi_{\ell}^{i+1}=-\stackrel{\circ}{\sigma}_{\ell}^{i+1} \psi_{\ell}^{i+1}$, we have

$$
\stackrel{\circ}{\sigma}_{\ell}^{1} \breve{\tau}_{j}^{0} \geq \stackrel{\circ}{\sigma}_{\ell}^{i+1} \breve{\tau}_{j}^{i}=\frac{\left(\stackrel{\circ}{\ell}_{\ell}^{i+1} \psi_{\ell}^{i+1}\right)^{2} \breve{\tau}_{j}^{i}}{\dot{\sigma}_{\ell}^{i+1}\left(\psi_{\ell}^{i+1}\right)^{2}}=\frac{\left(\lambda_{j, \ell}^{i}\right)^{2} \stackrel{\circ}{\ell}_{\ell}^{i+1}}{\psi_{\ell}^{i+1} \phi_{j}^{i}} \quad(j=1, \ldots, m) .
$$

By the orthogonality of the projections $P_{j}$, we may insert this estimate into the second part of (5.2), obtaining (3.5); compare the proof of Theorem 3.5. The definition of $\overline{\mathcal{V}}_{j}^{i}(\ell)$ in (3.3) also reduces to that in (5.3b), while the definition of $w_{j, \ell}^{i}$ in (5.3a) is exactly that in (3.4). We finish by applying Theorem 3.5 to verify (3.6).

REMARK 5.2. The first part of (5.2) is a relaxation of the property $\tau^{i} \sigma^{i+1}=\tau^{0} \sigma^{1}$ that would be satisfied by a dual-first variant of the basic PDPS; compare Theorem 4.2. 


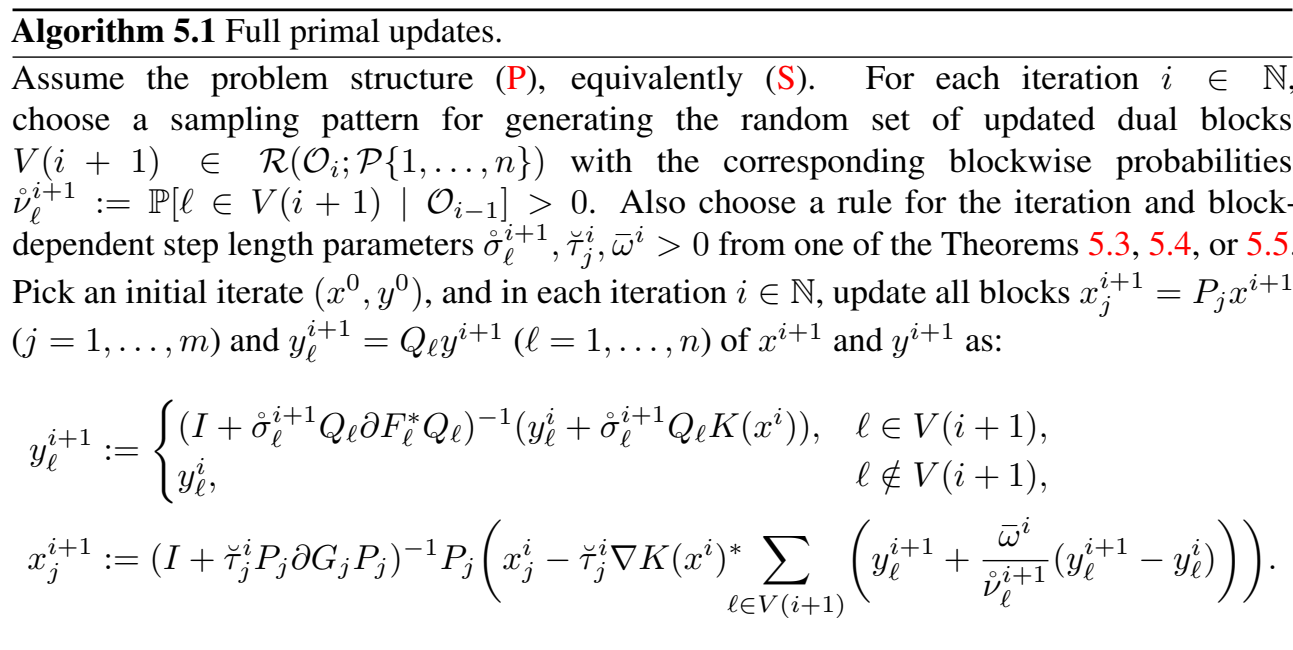

Finally, we also remind that for this section, (3.27) and (3.28) simplify to

$$
\bar{\gamma}_{G K, j}^{i} \equiv \gamma_{G, j}+\gamma_{K, j}-\alpha_{x}
$$

and

$$
\bar{\gamma}_{F^{*}, \ell}^{i+1} \equiv \bar{\gamma}_{F^{*}, \ell}:= \begin{cases}\gamma_{F^{*}, \ell}, & Q_{\ell} P_{\mathrm{NL}}=0 \\ \gamma_{F^{*}, \ell}-(p-1) \zeta_{\ell}, & Q_{\ell} P_{\mathrm{NL}} \neq 0\end{cases}
$$

5.1. Accelerated rates. As in Section 4 , we start with simple step length rules that yield $O(1 / N)$-convergence rates for those blocks that exhibit second-order growth.

THEOREM 5.3. Suppose that Assumption 3.1, 3.2, and 3.4 hold with $L, L_{3} \geq 0$, $p \in[1,2], \gamma_{G, j}+\gamma_{K, j}>0(j=1, \ldots, m)$, and $\gamma_{F^{*}, \ell} \geq(p-1) \zeta_{\ell}$, for some $\zeta_{\ell} \geq 0$ when $Q_{\ell} P_{\mathrm{NL}} \neq 0(\ell=1, \ldots, n)$. Let the iterates $\left\{u^{i}=\left(x^{i}, y^{i}\right)\right\}_{i \in \mathbb{N}}$ be generated by Algorithm 5.1 with iteration-independent probabilities $\stackrel{\nu}{\nu}_{\ell}^{i} \equiv \stackrel{\circ}{\nu}_{\ell}$ and step lengths

$$
\stackrel{\circ}{\sigma}_{\ell}^{i+1}:=\frac{\stackrel{\circ}{\sigma}_{\ell}^{i}}{1+2 \dot{\circ}_{\ell}^{i} \widetilde{\gamma}_{F^{*}, \ell}}, \quad \bar{\omega}^{i} \equiv 1, \quad \text { and } \quad \breve{\tau}_{j}^{i+1}:=\frac{\breve{\tau}_{j}^{i}}{1+2 \breve{\tau}_{j}^{i} \widetilde{\gamma}_{G, j}}
$$

with $0 \leq \widetilde{\gamma}_{G, j}<\gamma_{G, j}+\gamma_{K, j}(j=1, \ldots, m)$ and either $0 \leq \widetilde{\gamma}_{F^{*}, \ell}<\stackrel{\circ}{\nu}_{\ell} \bar{\gamma}_{F^{*}, \ell}$ or $\widetilde{\gamma}_{F^{*}, \ell}=\bar{\gamma}_{F^{*}, \ell}=0$ for each $\ell=1, \ldots, n$, and $\bar{\gamma}_{F^{*}, \ell}$ defined in (5.4). Moreover, suppose that the initial $\widetilde{\tau}_{j}^{0}, \stackrel{\circ}{\ell}_{\ell}^{1}>0$ satisfy, for some $\rho_{\ell} \geq 0(\ell=1, \ldots, n), 0<\delta<\kappa<1$ and with $w_{j, \ell}^{i}$ as in (5.3), the bounds

$$
\begin{aligned}
1-\kappa & \geq\left\|\sum_{j=1}^{m} \sqrt{\frac{w_{j, \ell}^{i} \dot{\sigma}_{\ell}^{1} \tilde{\tau}_{j}^{0}}{\nu_{\ell}}} Q_{\ell} \nabla K\left(x^{i}\right) P_{j}\right\|^{2}, \\
\delta & \geq \breve{\tau}_{j}^{0}\left(L_{3}+\frac{m L^{2}}{2 \min _{j=1, \ldots, m}\left(\gamma_{G, j}+\gamma_{K, j}-\widetilde{\gamma}_{G, j}\right)} \sum_{\ell=1}^{n} \rho_{\ell}^{2}\right), \quad \text { and } \\
\frac{\kappa-\delta}{1-\delta} & \geq 2 \chi_{V(i+1)}(\ell)\left(1-\stackrel{\nu}{\ell} \ell_{\ell}\right) \bar{\gamma}_{F^{*}, \ell} \dot{\circ}_{\ell}^{1} \frac{\bar{\gamma}_{F^{*}, \ell}-\widetilde{\gamma}_{F^{*}, \ell}}{\nu_{\ell} \bar{\gamma}_{F^{*}, \ell}-\widetilde{\gamma}_{F^{*}, \ell}} \quad(i \in \mathbb{N} ; j=1, \ldots, m) .
\end{aligned}
$$




\section{ETNA}

Kent State University and

Johann Radon Institute (RICAM)

Assume that

$$
\begin{aligned}
\theta_{I} & \geq p^{-p} \sum_{\ell=1}^{n}\left(\stackrel{\circ}{\ell} \ell^{2} \zeta_{\ell}^{1-p} \rho_{\ell}^{2-p} \quad\right. \text { and } \\
1 & =\mathbb{P}\left[\left\|Q_{\ell}\left(y^{i+1}-\widehat{y}\right)\right\|_{P_{\mathrm{NL}}} \leq \rho_{\ell},(\ell=1, \ldots, n) \mid \mathcal{O}_{i-1}\right] .
\end{aligned}
$$

Then $\mathbb{E}\left[\left\|P_{j}\left(x^{N}-\widehat{x}\right)\right\|^{2}\right] \rightarrow 0$ at the rate $O(1 / N)$ for all $j$ such that $\widetilde{\gamma}_{G, j}>0$ and $\mathbb{E}\left[\left\|Q_{\ell}\left(y^{N}-\widehat{y}\right)\right\|^{2}\right] \rightarrow 0$ at the rate $O(1 / N)$ for all $\ell$ such that $\widetilde{\gamma}_{F^{*}, \ell}>0$.

Proof. We will use Theorem 3.11, whose conditions we need to verify. With the choice of $\stackrel{\circ}{S}(i)=\emptyset, S(i)=\{1, \ldots, m\}$, and $\stackrel{\circ}{V}(i+1)=V(i+1)$ in Algorithm 5.1, we have already verified the nesting conditions (2.9) and reduced the coupling conditions (3.24) to (5.1). To verify (5.1), we set $\phi_{j}^{0}=\eta^{1} / \breve{\tau}_{j}^{0}, \psi_{\ell}^{2}=\eta^{1} /\left(\stackrel{\circ}{\sigma}_{\ell}^{2} \stackrel{\nu}{\ell}_{\ell}\right)$ for some $\eta^{1}>0$ and update

$$
\phi_{j}^{i+1}=\left(1+2 \widetilde{\tau}_{j}^{i} \widetilde{\gamma}_{G, j}\right) \phi_{j}^{i}, \quad \psi_{\ell}^{i+2}=\left(1+2 \dot{\sigma}_{\ell}^{i+1} \widetilde{\gamma}_{F^{*}, \ell}\right) \psi_{\ell}^{i+1}, \quad \text { and } \quad \eta^{i+1}:=\eta^{i} .
$$

Then $\stackrel{\circ}{\ell}_{\ell}{ }^{i+2} \psi_{\ell}^{i+2}=\eta^{i+1}=\phi_{j}^{i} \breve{\tau}_{j}^{i}$ due to (5.5) for all $\ell$ and $j$, and (5.1) follows. Clearly, also (3.14) holds because the step length and testing parameters are updated deterministically. The conditions (3.25) follow from (5.7) given that in Assumption 3.2 we can take $\theta_{\Phi_{i} T_{i}}=\eta^{i+1} \theta_{I}=\eta^{i} \theta_{I}=\psi_{\ell}^{i+1} \breve{\sigma}_{\ell}^{i+1} \theta_{I} / \stackrel{\nu}{\ell}_{\ell}$ and $\rho_{x}$ can be taken infinitely large.

The step length parameters $\dot{\sigma}^{i+1}$ and $\breve{\tau}_{j}^{i}$ are non-increasing in $i$ by the defining identities (5.5). Also using (5.6a), we thus verify (5.2). Hence Theorem 5.1 establishes (3.6).

We still need to verify Theorem 3.11 (i) and (ii). As far as the former is concerned, $\phi_{j}^{i+1} \leq\left(1+2 \widetilde{\tau}_{j}^{i} \widetilde{\gamma}_{G, j}\right) \phi_{j}^{i}$ follows from (5.8). Moreover, after applying (5.1), the identities (3.23) and (3.26) reduce to

$$
c_{*}^{i}=\frac{m L^{2} \eta^{i+1}}{2 \alpha_{x}} \sum_{\ell=1}^{n} \rho_{\ell}^{2} \quad \text { and } \quad L_{j}^{i}=L_{3}+\frac{m L^{2}}{2 \alpha_{x}} \sum_{\ell=1}^{n} \rho_{\ell}^{2} .
$$

Thus, by setting $\alpha_{x}=\min _{j=1, \ldots, m}\left(\gamma_{G, j}+\gamma_{K, j}-\widetilde{\gamma}_{G, j}\right)>0$, Theorem 3.11 (i) option (a) follows for every $j$ from (5.6b) and $\breve{\tau}_{j}^{i+1}$ being non-increasing. Regarding the dual test, we have $\psi_{\ell}^{i+2} \leq\left(1+2 \dot{\sigma}_{\ell}^{i+1} \widetilde{\gamma}_{F^{*}, \ell}^{i+1}\right) \psi_{\ell}^{i+1}$, which together with (5.6c) leads to (3.40b). Therefore, Theorem 3.11 (ii) option (b) holds for every $\ell$.

We can now apply Theorem 3.11 to obtain (3.29). From (5.8) we have

$$
\begin{aligned}
& \phi_{j}^{i+1}=\phi_{j}^{i}+2 \widetilde{\gamma}_{G, j} \eta^{i+1}=\phi_{j}^{i}+2 \widetilde{\gamma}_{G, j} \eta^{1}=\ldots=\phi_{j}^{0}+2 i \widetilde{\gamma}_{G, j} \eta^{1} \text { and } \\
& \psi_{\ell}^{i+2}=\psi_{\ell}^{i+1}+2 \widetilde{\gamma}_{F^{*}, \ell} \eta^{i} / \stackrel{\circ}{\ell}_{\ell}=\psi_{\ell}^{i+1}+2 \widetilde{\gamma}_{F^{*}, \ell} \eta^{1} / \stackrel{\circ}{\ell}_{\ell}=\ldots=\psi_{\ell}^{1}+2(i+1) \widetilde{\gamma}_{F^{*}, \ell} \eta^{1} / \stackrel{\circ}{\ell}_{\ell} .
\end{aligned}
$$

Therefore, for any primal block $j$ with $\widetilde{\gamma}_{G, j}>0$ and dual block $\ell$ with $\widetilde{\gamma}_{F^{*}, \ell}>0, \phi_{j}^{N}$ and $\psi_{\ell}^{N+1}$ grow as $\Omega(N)$, respectively. This together with (3.29) gives the claim.

We get improved $O\left(1 / N^{2}\right)$-rates if all primal blocks exhibit second-order growth:

TheOrem 5.4. Suppose that Assumption 3.1, 3.2, and 3.4 hold with $L, L_{3} \geq 0$, $p \in[1,2], \gamma_{G, j}+\gamma_{K, j}>0(j=1, \ldots, m)$ and $\gamma_{F^{*}, \ell} \geq(p-1) \zeta_{\ell}$, for some $\zeta_{\ell}$ when $Q_{\ell} P_{\mathrm{NL}} \neq 0(\ell=1, \ldots, n)$. Let the iterates $\left\{u^{i}=\left(x^{i}, y^{i}\right)\right\}_{i \in \mathbb{N}}$ be generated by Algorithm 5.1 with iteration-independent probabilities $\stackrel{\circ}{\nu}_{\ell}^{i} \equiv \stackrel{\circ}{\nu}_{\ell}$ and step length parameters

$$
\stackrel{\circ}{\sigma}_{\ell}^{i+2}=\frac{{\stackrel{\circ}{\sigma_{\ell}}}^{i+1}}{\bar{\omega}^{i}}, \quad \breve{\tau}_{j}^{i+1}=\frac{1}{1+2 \breve{\tau}_{j}^{i} \widetilde{\gamma}_{G, j}} \frac{\breve{\tau}_{j}^{i}}{\overline{\bar{\omega}}^{i+1}}, \quad \text { and } \quad \bar{\omega}^{i+1}:=\max _{j=1, \ldots, m} \frac{1}{\sqrt{1+2 \breve{\tau}_{j}^{i} \widetilde{\gamma}_{G, j}}},
$$

with $0<\widetilde{\gamma}_{G, j}<\gamma_{G, j}+\gamma_{K, j}(j=1, \ldots, m)$. Moreover, suppose that the initial $\bar{\omega}^{0}=1, \breve{\tau}_{j}^{0}$ and $\stackrel{\circ}{\ell}_{\ell}^{1}$ satisfy, for some $\rho_{\ell} \geq 0(\ell=1, \ldots, n), 0<\delta \leq \kappa<1$ and with $w_{j, \ell}^{i}$ as in (5.3), the 
bounds

$$
\begin{aligned}
& 1-\kappa \geq\left\|\sum_{j=1}^{m} \sqrt{\frac{w_{j, \ell}^{i} \stackrel{\circ}{\sigma}_{\ell}^{1} \breve{\tau}_{j}^{0}}{\stackrel{\circ}{\nu}_{\ell}}} Q_{\ell} \nabla K\left(x^{i}\right) P_{j}\right\|^{2} \quad \text { and } \\
& \delta \geq \breve{\tau}_{j}^{0}\left(L_{3}+\frac{m L^{2}}{2 \min _{j=1, \ldots, m}\left(\gamma_{G, j}+\gamma_{K, j}-\widetilde{\gamma}_{G, j}\right)} \sum_{\ell=1}^{n} \rho_{\ell}^{2}\right) \\
& (i \in \mathbb{N} ; j=1, \ldots, m) .
\end{aligned}
$$

Also assume

$$
\begin{aligned}
\theta_{I} & \geq p^{-p} \sum_{\ell=1}^{n}\left(\stackrel{\circ}{\ell} \ell^{2} \zeta_{\ell}^{1-p} \rho_{\ell}^{2-p} \quad\right. \text { and } \\
1 & =\mathbb{P}\left[\left\|Q_{\ell}\left(y^{i+1}-\widehat{y}\right)\right\|_{P_{\mathrm{NL}}} \leq \rho_{\ell}, \quad(\ell=1, \ldots, n) \mid \mathcal{O}_{i-1}\right] .
\end{aligned}
$$

Then $\mathbb{E}\left[\left\|P_{j}\left(x^{N}-\widehat{x}\right)\right\|^{2}\right] \rightarrow 0$ at the rate $O\left(1 / N^{2}\right)$ for all $j$.

Proof. We will use Theorem 3.11 whose conditions we need to verify. With the choice of $\stackrel{\circ}{S}(i)=\emptyset, S(i)=\{1, \ldots, m\}$, and $\stackrel{\circ}{V}(i+1)=V(i+1)$ in Algorithm 5.1, we have already verified the nesting conditions (2.9) and reduced the coupling conditions (3.24) to (5.1). To verify (5.1), we set $\phi_{j}^{0}=\eta^{1} / \breve{\tau}_{j}^{0}$ an $\psi_{\ell}^{2}:=\eta^{1} /\left(\stackrel{\circ}{\nu} \ell_{\ell}{ }^{2}\right)$ for some $\eta^{1}>0$ and update

$$
\phi_{j}^{i+1}:=\left(1+2 \breve{\tau}_{j}^{i} \widetilde{\gamma}_{G, j}\right) \phi_{j}^{i}, \quad \psi_{\ell}^{i+1}:=\psi_{\ell}^{i}, \quad \text { and } \quad \eta^{i+1}=\eta^{i} / \bar{\omega}^{i} .
$$

Then from (5.9), we inductively get $\stackrel{\circ}{\nu}_{\ell} \psi_{\ell}^{i+2} \stackrel{\circ}{\sigma}_{\ell}^{i+2}=\stackrel{\circ}{\nu}_{\ell} \psi_{\ell}^{i+1} \stackrel{\circ}{\sigma}_{\ell}^{i+1} / \bar{\omega}^{i}=\eta^{i+1}$ for all $\ell$. From (5.9), we also have inductively for all $j$ that $\phi_{j}^{i+1} \breve{\tau}_{j}^{i+1}=\phi_{j}^{i} \breve{\tau}_{j}^{i} / \bar{\omega}^{i+1}=\eta^{i+2}$. Therefore, (5.1) holds. Then, conditions (3.25) follow from (5.11) given that $\bar{\omega}^{i} \leq 1$ and in Assumption 3.2 we can take $\theta_{\Phi_{i} T_{i}}=\eta^{i+1} \theta_{I}=\eta^{i} \theta_{I} / \bar{\omega}^{i}=\psi_{\ell}^{i+1} \sigma_{\ell}^{i+1} \theta_{I} /\left(\nu_{\ell} \bar{\omega}^{i}\right)$ and $\rho_{x}$ can be taken infinitely large. Clearly, also (3.14) holds because the step length and testing parameters are updated deterministically.

We now verify (3.6). From (5.9) we obtain

$$
\begin{aligned}
& \dot{\sigma}_{\ell}^{i+2} \breve{\tau}_{j}^{i+1}=\frac{\stackrel{\circ}{\sigma}_{\ell}^{i+1} \breve{\tau}_{j}^{i}}{\bar{\omega}^{i} \bar{\omega}^{i+1}\left(1+2 \widetilde{\gamma}_{G, j} \breve{\tau}_{j}^{i}\right)} \leq \stackrel{\circ}{\sigma}_{\ell}^{i+1} \breve{\tau}_{j}^{i} \sqrt{\frac{1+2 \widetilde{\gamma}_{G, j} \breve{\tau}_{j}^{i-1}}{1+2 \widetilde{\gamma}_{G, j} \breve{\tau}_{j}^{i}}} \\
& \leq \ldots \leq \dot{\sigma}_{\ell}^{2} \breve{\tau}_{j}^{1} \sqrt{\frac{1+2 \widetilde{\gamma}_{G, j} \breve{\tau}_{j}^{0}}{1+2 \widetilde{\gamma}_{G, j} \breve{\tau}_{j}^{i}}} \\
& =\stackrel{\circ}{\sigma}_{\ell}^{1} \breve{\tau}_{j}^{0} \frac{1}{\bar{\omega}^{1} \sqrt{1+2 \widetilde{\gamma}_{G, j} \breve{\tau}_{j}^{0}}} \frac{1}{1+2 \widetilde{\gamma}_{G, j} \breve{\tau}_{j}^{i}} \leq \stackrel{\circ}{\sigma}_{\ell}^{1} \breve{\tau}_{j}^{0} .
\end{aligned}
$$

This and (5.10) verify (5.2). Thus, Theorem 5.1 establishes (3.6).

We still need to verify Theorem 3.11 (i) and (ii). Regarding the former, the inequality $\phi_{j}^{i+1} \leq\left(1+2 \widetilde{\tau}_{j}^{i} \widetilde{\gamma}_{G, j}\right) \phi_{j}^{i}$ follows from (5.12). Moreover, after applying (5.1), equalities (3.23) and (3.26) reduce to

$$
c_{*}^{i}=\frac{m L^{2} \eta^{i+1}}{2 \alpha_{x}} \sum_{\ell=1}^{n} \rho_{\ell}^{2} \quad \text { and } \quad L_{j}^{i}=L_{3}+\frac{m L^{2}}{2 \alpha_{x}} \sum_{\ell=1}^{n} \rho_{\ell}^{2} .
$$

Thus, by setting $\alpha_{x}=\min _{j=1, \ldots, m}\left(\gamma_{G, j}+\gamma_{K, j}-\widetilde{\gamma}_{G, j}\right)>0$, Theorem 3.11 (i) option (a) follows for every $j$ from the second inequality in (5.10) and $\widetilde{\tau}_{j}^{i+1}$ being decreasing. As for 
Theorem 3.11 (ii), $\psi_{\ell}^{i+1}=\psi_{\ell}^{i+2} \leq\left(1+2 \chi_{V(i+1)}(\ell) \sigma_{\ell}^{i+1} \bar{\gamma}_{F^{*}, \ell}^{i+1}\right) \psi_{\ell}^{i+1}$ holds trivially as we have assumed $\bar{\gamma}_{F^{*}, \ell}^{i+1} \geq 0$. Thus, Theorem 3.11 (ii) option (a) holds for every $\ell$.

We can now use Theorem 3.11 to verify (3.29). Multiplying the $\tau$-update of (5.9) by $2 \widetilde{\gamma}_{G, j}$, plugging in $\bar{\omega}^{i+1}$, and taking the inverse, we get

$$
\begin{aligned}
\left(2 \breve{\tau}_{j}^{i+1} \widetilde{\gamma}_{G, j}\right)^{-1} & =\frac{1+2 \breve{\tau}_{j}^{i} \widetilde{\gamma}_{G, j}}{2 \breve{\tau}_{j}^{i} \widetilde{\gamma}_{G, j} \sqrt{1+\min _{j=1, \ldots, m}\left(2 \breve{\tau}_{j}^{i} \widetilde{\gamma}_{G, j}\right)}} \\
& =\frac{1+\left(2 \breve{\tau}_{j}^{i} \widetilde{\gamma}_{G, j}\right)^{-1}}{\sqrt{1+\left(\max _{j=1, \ldots, m}\left(2 \breve{\tau}_{j}^{i} \widetilde{\gamma}_{G, j}\right)^{-1}\right)^{-1}}} .
\end{aligned}
$$

We then apply Theorem B.1 with $z_{j}^{i}=\left(2 \widetilde{\tau}_{j}^{i} \widetilde{\gamma}_{G, j}\right)^{-1}$ to obtain

$$
\max _{j=1, \ldots, m}\left(2 \breve{\tau}_{j}^{N} \widetilde{\gamma}_{G, j}\right)^{-1} \leq \bar{z}_{0}+N / 2
$$

with $\bar{z}_{0}>0$. Then, from (5.12), we have

$$
\begin{aligned}
\phi_{j}^{N+1} & \geq\left(1+\min _{j=1, \ldots, m}\left(2 \breve{\tau}_{j}^{i} \widetilde{\gamma}_{G, j}\right)\right) \phi_{j}^{N} \geq\left(1+\frac{1}{\bar{z}_{0}+N / 2}\right) \phi_{j}^{N}=\frac{2 \bar{z}_{0}+N+2}{2 \bar{z}_{0}+N} \phi_{j}^{N} \\
& =\frac{2 \bar{z}_{0}+N+2}{2 \bar{z}_{0}+N} \frac{2 \bar{z}_{0}+N+1}{2 \bar{z}_{0}+N-1} \phi_{j}^{N-1}=\ldots=\frac{\left(2 \bar{z}_{0}+N+2\right)\left(2 \bar{z}_{0}+N+1\right)}{2 \bar{z}_{0}\left(2 \bar{z}_{0}+1\right)} \phi_{j}^{0} .
\end{aligned}
$$

Therefore, $\phi_{j}^{N}$ grows as $\Omega\left(N^{2}\right)$. We obtain the claimed convergence rates from (3.29).

5.2. Linear convergence. If all the primal and dual blocks exhibit second-order growth, i.e., $\bar{\gamma}_{F^{*}, \ell}>0$ and $\gamma_{G, j}+\gamma_{K, j}>0$, then we obtain linear convergence:

THEOREM 5.5. Suppose that Assumptions 3.1, 3.2, and 3.4 hold with $L, L_{3} \geq 0$, $p \in[1,2], \gamma_{G, j}+\gamma_{K, j}>0(j=1, \ldots, m)$. Let the iterates $\left\{u^{i}=\left(x^{i}, y^{i}\right)\right\}_{i \in \mathbb{N}}$ be generated by Algorithm 5.1 with iteration-independent $\stackrel{\nu}{\ell}_{\ell}^{i} \equiv \stackrel{\nu}{\ell}_{\ell}$ and step lengths

$$
\begin{aligned}
\breve{\tau}_{j}^{i+1} & :=\frac{\breve{\tau}_{j}^{i}}{\left(1+2 \breve{\tau}_{j}^{i} \widetilde{\gamma}_{G, j}\right) \bar{\omega}}, \quad \stackrel{\sigma}{\sigma}_{\ell}^{i+2}:=\frac{\dot{\sigma}_{\ell}^{i+1}}{\left(1+2 \dot{\sigma}_{\ell}^{i+1} \widetilde{\gamma}_{\left.F^{*}, \ell\right) \bar{\omega}}\right.}, \quad \text { and } \\
\bar{\omega}^{i} & \equiv \bar{\omega}:=\max \left\{\max _{j=1, \ldots, m} \frac{1}{1+2 \breve{\tau}_{j}^{0} \widetilde{\gamma}_{G, j}}, \max _{\ell=1, \ldots, n} \frac{1}{1+2 \dot{\sigma}_{\ell}^{1} \widetilde{\gamma}_{F^{*}, \ell}}\right\}
\end{aligned}
$$

with $0<\widetilde{\gamma}_{G, j}<\gamma_{G, j}+\gamma_{K, j}(j=1, \ldots, m)$ and $0<\widetilde{\gamma}_{F^{*}, \ell}<\stackrel{\circ}{\ell} \ell_{F_{F^{*}, \ell}}(\ell=1, \ldots, n)$, and $\bar{\gamma}_{F^{*}, \ell}$ defined in (5.4). Moreover, let the initial $\breve{\tau}_{j}^{0}, \stackrel{\circ}{\sigma}_{\ell}^{1}>0$ satisfy, for some $0<\delta<\kappa<1$, $\rho_{\ell} \geq 0(\ell=1, \ldots, n)$ and with $w_{j, \ell}^{i}$ as in (5.3), the bounds

$$
\begin{aligned}
& \text { (5.14a) } 1-\kappa \geq\left\|\sum_{j=1}^{m} \sqrt{\frac{w_{j, \ell}^{i} \stackrel{\circ}{\sigma}_{\ell}^{1} \breve{\tau}_{j}^{0}}{\check{\nu}_{\ell}}} Q_{\ell} \nabla K\left(x^{i}\right) P_{j}\right\|^{2}, \\
& \text { (5.14b) } \quad \delta \geq \breve{\tau}_{j}^{0}\left(L_{3}+\frac{m L^{2}}{2 \min _{j=1, \ldots, m}\left(\gamma_{G, j}+\gamma_{K, j}-\widetilde{\gamma}_{G, j}\right)} \sum_{\ell=1}^{n} \rho_{\ell}^{2}\right), \quad \text { and } \\
& \text { (5.14c) } \frac{\kappa-\delta}{1-\delta} \geq 2(1-\stackrel{\circ}{\nu})_{\ell} \bar{\gamma}_{F^{*}, \ell} \stackrel{\circ}{\sigma}_{\ell}^{1} \frac{\bar{\gamma}_{F^{*}, \ell}-\widetilde{\gamma}_{F^{*}, \ell}}{\nu_{\ell} \bar{\gamma}_{F^{*}, \ell}-\widetilde{\gamma}_{F^{*}, \ell}} \\
& (\ell \in V(i+1) ; j=1, \ldots, m ; i \in \mathbb{N}) .
\end{aligned}
$$


Further assume that

$$
\begin{aligned}
\theta_{I} & \geq p^{-p} \bar{\omega} \sum_{\ell=1}^{n}\left(\grave{\nu}_{\ell}\right)^{2} \zeta_{\ell}^{1-p} \rho_{\ell}^{2-p} \quad \text { and } \\
1 & =\mathbb{P}\left[\left\|Q_{\ell}\left(y^{i+1}-\widehat{y}\right)\right\|_{P_{\mathrm{NL}}} \leq \rho_{\ell},(\ell=1, \ldots, n) \mid \mathcal{O}_{i-1}\right] .
\end{aligned}
$$

Then $\mathbb{E}\left[\left\|P_{j}\left(x^{N}-\widehat{x}\right)\right\|^{2}\right] \rightarrow 0$ and $\mathbb{E}\left[\left\|Q_{\ell}\left(y^{N}-\widehat{y}\right)\right\|^{2}\right] \rightarrow 0$ at the linear rate $O\left((1 / \bar{\omega})^{N}\right)$ for all $j \in\{1, \ldots, m\}$ and $\ell \in\{1, \ldots, n\}$.

Proof. We will use Theorem 3.11, whose conditions we need to verify. With the choice of $\stackrel{\circ}{S}(i)=\emptyset, S(i)=\{1, \ldots, m\}$, and $\dot{V}(i+1)=V(i+1)$ in Algorithm 5.1, we have already verified the nesting conditions (2.9) and reduced the coupling conditions (3.24) to (5.1). To

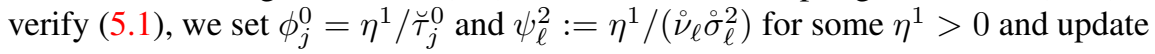

$$
\phi_{j}^{i+1}:=\left(1+2 \widetilde{\tau}_{j}^{i} \widetilde{\gamma}_{G, j}\right) \phi_{j}^{i}, \quad \psi_{\ell}^{i+1}:=\left(1+2 \dot{\sigma}_{\ell}^{i} \widetilde{\gamma}_{F^{*}, \ell}\right) \psi_{\ell}^{i}, \quad \text { and } \quad \eta^{i+1}=\eta^{i} / \bar{\omega} .
$$

Then from (5.13), we inductively get $\stackrel{\circ}{\nu}_{\ell} \psi_{\ell}^{i+2} \stackrel{\circ}{\sigma}_{\ell}^{i+2}=\stackrel{\circ}{\nu}_{\ell} \psi_{\ell}^{i+1}{\stackrel{\circ}{\sigma_{\ell}}}_{\ell} / \bar{\omega}=\eta^{i+1}$ for all $\ell$ and $\phi_{j}^{i+1} \breve{\tau}_{j}^{i+1}=\phi_{j}^{i} \breve{\tau}_{j}^{i} / \bar{\omega}=\eta^{i+2}$ for all $j$. Therefore, (5.1) holds. Then, conditions (3.25) follow from (5.15) given that in Assumption 3.2 we can take

$$
\theta_{\Phi_{i} T_{i}}=\eta^{i+1} \theta_{I}=\eta^{i} \theta_{I} / \bar{\omega}=\psi_{\ell}^{i+1} \sigma_{\ell}^{i+1} \theta_{I} /\left(\stackrel{\circ}{\nu}_{\ell} \bar{\omega}\right)
$$

and $\rho_{x}$ can be taken infinitely large. Clearly, also (3.14) holds because the step length and testing parameters are updated deterministically.

We now verify (3.6). We start by proving by induction that

$$
\bar{\omega}=\max \left\{\max _{j=1, \ldots, m} \frac{1}{1+2 \widetilde{\tau}_{j}^{i} \widetilde{\gamma}_{G, j}}, \max _{\ell=1, \ldots, n} \frac{1}{1+2 \dot{\circ}_{\ell}^{i+1} \widetilde{\gamma}_{F^{*}, \ell}}\right\},
$$

in other words,

$$
\bar{\omega}^{-1}=1+\min \left\{\min _{j=1, \ldots, m} 2 \breve{\tau}_{j}^{i} \widetilde{\gamma}_{G, j}, \min _{\ell=1, \ldots, n} 2 \stackrel{\sigma}{\sigma}_{\ell}^{i+1} \widetilde{\gamma}_{F^{*}, \ell}\right\} .
$$

The inductive base for $i=0$ holds by (5.13b). Using (5.13a) yields

$$
\begin{aligned}
& \min \left\{\min _{j=1, \ldots, m} 2 \breve{\tau}_{j}^{i+1} \widetilde{\gamma}_{G, j}, \min _{\ell=1, \ldots, n} 2 \dot{\sigma}_{\ell}^{i+2} \widetilde{\gamma}_{F^{*}, \ell}\right\} \\
& =\frac{1}{\bar{\omega}} \min \left\{\min _{j=1, \ldots, m} \frac{1}{1+\left(2 \widetilde{\tau}_{j}^{i} \widetilde{\gamma}_{G, j}\right)^{-1}}, \min _{\ell=1, \ldots, n} \frac{1}{1+\left(2 \sigma_{\ell}^{i+1} \widetilde{\gamma}_{F^{*}, \ell}\right)^{-1}}\right\} \\
& =\frac{1}{\bar{\omega}} \frac{1}{1+\min ^{-1}\left\{\min _{j=1, \ldots, m} 2 \breve{\tau}_{j}^{i} \widetilde{\gamma}_{G, j}, \min _{\ell=1, \ldots, n} 2 \dot{\sigma}_{\ell}^{i+1} \widetilde{\gamma}_{F^{*}, \ell}\right\}} \\
& =\min \left\{\min _{j=1, \ldots, m} 2 \breve{\tau}_{j}^{i} \widetilde{\gamma}_{G, j}, \min _{\ell=1, \ldots, n} 2 \dot{\sigma}_{\ell}^{i+1} \widetilde{\gamma}_{F^{*}, \ell}\right\} \text {. }
\end{aligned}
$$

This establishes the inductive step, hence (5.17), which in turn shows that $\breve{\tau}_{j}^{i}$ and $\stackrel{\circ}{\ell}_{\ell}^{i+1}$ as updated according to (5.13a) are non-increasing in $i$. Also using (5.14) proves (5.2). Thus, Theorem 5.1 verifies (3.6).

We need to verify Theorem 3.11 (i) and (ii). As for the former, (3.23) and (3.26) reduce to

$$
c_{*}^{i}=\frac{m L^{2}}{2 \alpha_{x}} \sum_{\ell=1}^{n} \rho_{\ell}^{2} \eta^{i+1} \quad \text { and } \quad L_{j}^{i}=L_{3}+\frac{m L^{2}}{2 \alpha_{x}} \sum_{\ell=1}^{n} \rho_{\ell}^{2},
$$




\section{ETNA}

Kent State University and

Johann Radon Institute (RICAM)

so (5.14) together with the non-increasing $\breve{\tau}_{j}^{i}$ and the update rule for $\phi_{j}^{i+1}$ in (5.16) verify Theorem 3.11 (i) option (a) for every $j$ and $\alpha_{x}=\min _{j=1, \ldots, m}\left(\gamma_{G, j}+\gamma_{K, j}-\widetilde{\gamma}_{G, j}\right)$. Regarding the latter, since we take $\widetilde{\gamma}_{F^{*}, \ell}<\grave{\nu}_{\ell} \bar{\gamma}_{F^{*}, \ell}$, we obtain (3.40b) using the last inequality of (5.14) and that $\stackrel{\circ}{\ell}_{\ell}^{i+1}$ is non-increasing by definition in (5.13). Hence, Theorem 3.11 (ii) option (b) holds for every $\ell$.

Therefore, we can apply Theorem 3.11 to obtain (3.29). By (5.16) and (5.17),

$$
\begin{aligned}
& \phi_{j}^{N+1}=\left(1+2 \breve{\tau}_{j}^{N} \widetilde{\gamma}_{G, j}\right) \phi_{j}^{N} \geq \phi_{j}^{N} / \bar{\omega} \geq \ldots \geq \phi_{j}^{0} / \bar{\omega}^{N+1} \quad \text { and } \\
& \psi_{\ell}^{N+1}=\left(1+2 \dot{\sigma}_{\ell}^{N} \widetilde{\gamma}_{F^{*}, \ell}\right) \psi_{\ell}^{N} \geq \psi_{\ell}^{N} / \bar{\omega} \geq \ldots \geq \psi_{\ell}^{1} / \bar{\omega}^{N} .
\end{aligned}
$$

Applying these estimates in (3.29) establishes the claimed linear convergence rates.

REMARK 5.6 (Stochastic sum-sampling forward-backward splitting). Consider the problem (1.1) with $F^{*}(y)=\delta_{\{\overrightarrow{1}\}}$ for $\overrightarrow{1}:=(1, \ldots, 1) \in \mathbb{R}^{n}$ and $\nabla K(x)^{*} y=\sum_{\ell=1}^{n} \nabla J_{\ell}(x) y_{(\ell)}$ with $y=\left(y_{(1)}, \ldots, y_{(n)}\right)$. Taking $Q_{\ell} y:=\left(0, \ldots, 0, y_{(\ell)}, 0, \ldots, 0\right)$, it follows that

$$
\left(I+\breve{\sigma}_{\ell}^{i+1} Q_{\ell} \partial F_{\ell}^{*} Q_{\ell}\right)^{-1} \equiv(0, \ldots, 0,1,0, \ldots, 0) .
$$

Consequently $y^{i} \equiv \overrightarrow{1}$ in all iterations, so that with just a single primal block with corresponding step length $\breve{\tau}^{i}=\breve{\tau}_{1}^{i}$, Algorithm 5.1 reduces to

$$
x^{i+1}:=\left(I+\breve{\tau}^{i} \partial G\right)^{-1}\left(x^{i}-\breve{\tau}^{i} \sum_{\ell \in V(i+1)} \nabla J_{\ell}\left(x^{i}\right)\right) .
$$

With random $V(i+1)$, this is a forward-backward splitting method that stochastically samples $\sum_{\ell} J_{\ell}$ in (1.1). We can take any $\gamma_{F^{*}, \ell} \in(0, \infty)$, which in Theorems 5.3-5.5 also allows us to take $\zeta_{\ell}$ arbitrarily large and $\stackrel{\circ}{\sigma}_{\ell}^{i}>0$ arbitrarily small. Consequently, the systems of step length bounds (5.6) and (5.14) reduce to their second part (with first and third part unnecessary), and (5.10) reduces to its second part. In other words, we only need to choose $\breve{\tau}^{0}$ sufficiently small.

6. Numerical experience. We will now study the performance of our proposed methods for two application problems: diffusion tensor imaging (DTI), which is a form of magnetic resonance imaging (MRI), and electrical impedance tomography (EIT).

6.1. Diffusion tensor imaging. Diffusion tensor imaging is covered by the StejskalTanner equation: given a tensor field $x: \Omega \rightarrow \operatorname{Sym}^{2}\left(\mathbb{R}^{3}\right)$ associating each point on the domain $\Omega \subset \mathbb{R}^{3}$ with a symmetric 2 -tensor (presentable as a symmetric $3 \times 3$ matrix) and a non-diffusion-weighted image $s_{0}: \Omega \rightarrow \mathbb{R}$, then the diffusion-weighted image $s_{k}: \Omega \rightarrow \mathbb{R}$ corresponding to a diffusion-sensitising gradient $b_{k} \in \mathbb{R}^{3}$ is given by

$$
s_{k}(\xi)=s_{0}(\xi) e^{-\left\langle x(\xi) b_{k}, b_{k}\right\rangle} \quad(\xi \in \Omega) .
$$

At each spatial point $\xi$, the tensor $x(\xi)$ models the covariance of a Gaussian probability distribution for the spatial directions of the diffusion of water at that point. Models more advanced than DTI, such as HARDI, consider composite probability distributions at each $\xi$. For our purposes, a simplified DTI model will be sufficient. One can measure $s_{k}$ and $s_{0}$ by suitable MRI pulse sequences, the inversion of a Fourier transform, and taking the absolute value of a complex number; for details we refer to [1,19] among others. We recommend [24] as an introduction to MRI.

We want to determine $x$ from noisy measurements of $s_{0}$ and $s_{k}(k=1, \ldots, N)$. Clearly, (6.1) can be converted into an invertible system of linear equations with respect to $x$ if $N \geq 6$ and the tensors $b_{k} \otimes b_{k}$ are linearly independent. With noise involved, to get a 


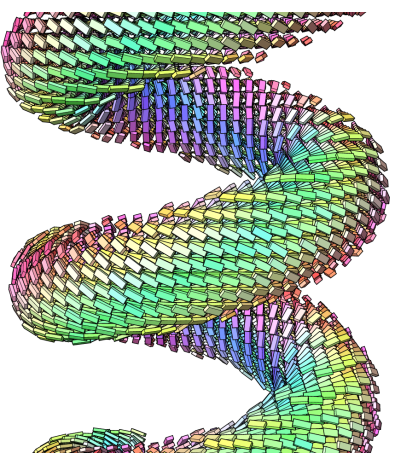

(a) Original helix.

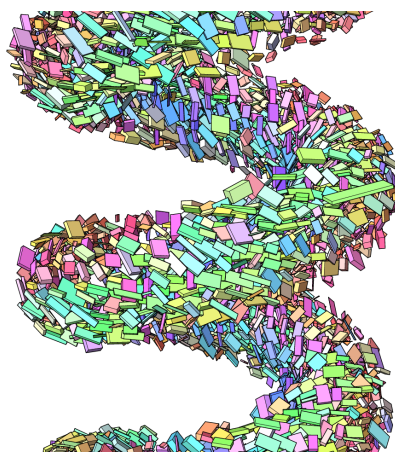

(b) Least squares reconstruction.

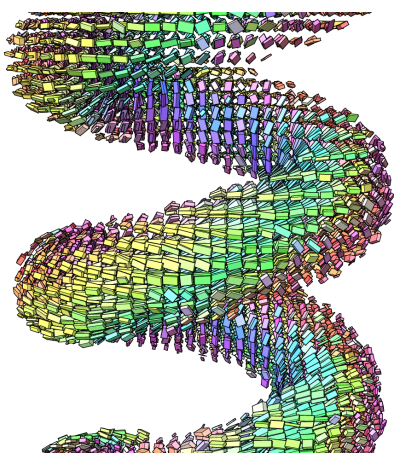

(c) Regularised reconstruction.

FIG. 6.1. Visualisation of original helix data (a) and the reconstruction from noisy diffusion-weighted measurements. The reference least-squares reconstruction in $(b)$ is based on linearising (6.1) with respect to $x$ by taking the logarithm. The regularised reconstruction (c) is the numerical solution of (6.2) for $\alpha=0.005$ with the variant (d2) of our method after 10000 iterations. The visualisation, generated with Teem [40], displays the tensor at each voxel of the $3 D$ volume as a cuboid oriented along the eigenvectors of the tensor with the size of each side proportional to the corresponding eigenvalue. The cuboids are also colour-coded based on the principal eigenvector. Tensors with too small eigenvalues are suppressed; in essence this suppresses the background outside the helix, letting the latter to be inspected unobstructedly.

good-quality image, we want to obtain a regularised solution. We therefore consider a problem of the form $\left(\mathrm{P}_{0}\right)$, where $G$ is a data term modelling (6.1) along with any noise and $F \circ K$ is the regulariser. Ideally, our data term would model the Rician noise distribution, which is the distribution of the absolute value of a complex number when the latter has Gaussian noise distribution. However, the numerical treatment of the Rician distribution is quite involved-we refer to $[17,20]$ for some variational approaches — and instead of modelling it directly, a more fruitful approach may be to work with complex data directly, even incorporating the Fourier transform into our model. For the purposes of the present work, since we only use synthetic data, we will therefore assume that the noise in $s_{k}$ is Gaussian. We note that (6.2) in infinite dimensions requires the use of the Banach space of functions of bounded deformation, so, since our algorithms require Hilbert spaces, only discretised versions of the model can be considered. Consequently, taking the discretised domain $\Omega_{d}:=\left\{1, \ldots, n_{1}\right\} \times\left\{1, \ldots, n_{2}\right\} \times\left\{1, \ldots, n_{3}\right\}$ and incorporating total deformation regularisation with parameter $\alpha>0$, we seek to solve

$$
\begin{aligned}
\min _{x: \Omega_{d} \rightarrow \operatorname{Sym}^{2}\left(\mathbb{R}^{3}\right)} & \frac{1}{2}\|T(x)\|^{2}+\alpha\left\|\mathcal{E}_{d} x\right\|_{F, 1}, \quad \text { where } \\
& {[T(x)]_{k}:=s_{k}(\xi)-s_{0}(\xi) e^{-\left\langle x(\xi) b_{k}, b_{k}\right\rangle} \quad(k=1, \ldots, N) . }
\end{aligned}
$$

Here $\left[\mathcal{E}_{d} x\right](\xi) \in \operatorname{Sym}^{3}\left(\mathbb{R}^{3}\right)$ is a forward-differences discretisation of the symmetrised gradient, a symmetric third-order tensor. The $F, 1$-norm is based on taking pointwisely the Frobenius norm of $\left[\mathcal{E}_{d} x\right](\xi)$ and an integration over the space variable (1-norm). This model is sightly simplified from our previous work in [37, 38, 39], where second-order total generalised variation regularisation was considered, and we included a positivity semi-definiteness constraint on $x(\xi)$.

To write (6.2) in the form (S), we take with $y=(\mu, \lambda)$ the functions

$$
\begin{gathered}
G(x):=0, \quad K(x):=\left(\mathcal{E}_{d} x, T(x)\right), \quad F^{*}(y):=F_{\mu}^{*}(\mu)+F_{\lambda}^{*}(\lambda), \\
F_{\mu}^{*}(\mu):=\delta_{\alpha \mathbb{B}}(\mu), F_{\lambda}^{*}(\lambda):=\frac{1}{2}\|\lambda\|^{2} .
\end{gathered}
$$




\section{ETNA}

Kent State University and

Johann Radon Institute (RICAM)

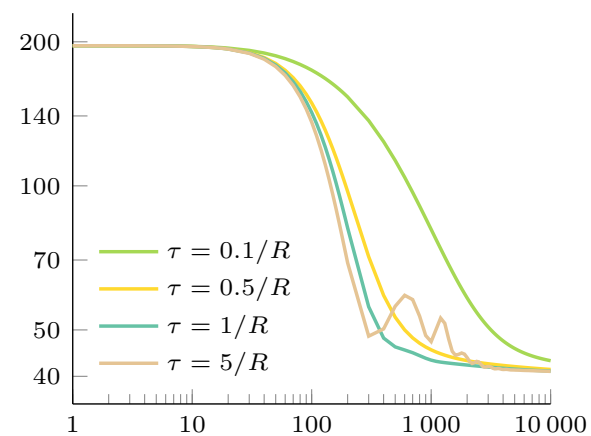

(a) Multiple step length parametrisations of the nonblock-adapted reference algorithm (d1) to justify the choice $\tau=1 / R$.

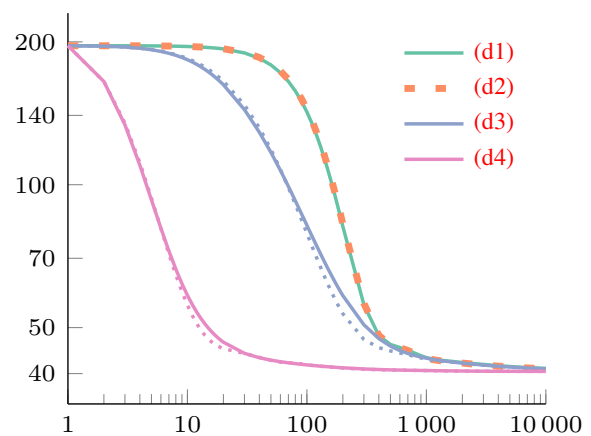

(b) Comparison of the algorithm variants (d1)-(d4). The dotted lines show the effect of accelerating the dual blocks in (d3) and (d4) following Theorem 4.4.

FIG. 6.2. Reference algorithm step length justification (a) and algorithm performance (b) for the DTI problem. Function values are on the vertical axis, and iteration counts are on the horizontal axis. Based on (a), we take $\tau=1 / R$ in (b); $\tau=5 / R$ appears to have convergence issues, and $\tau=0.5 / R$ yields slower convergence.

Here $\mathbb{B}$ is the product of the voxelwise unit balls of $\operatorname{Sym}^{3}\left(\mathbb{R}^{3}\right)$ over $\Omega_{d}$. To better satisfy the conditions of our convergence theorems, we replace $F_{\mu}^{*}$ by $F_{\mu, \gamma}^{*}(\mu):=\delta_{\alpha \mathbb{B}}(\mu)+\gamma \alpha^{-1}\|\mu\|^{2}$ with $\gamma=10^{-9}$. This is the same as applying Moreau-Yosida regularisation to \|\|$_{F, 1}$ in (6.2).

We generated our test data, a simple helix depicted in Figure 6.1, with the Teem toolkit [40]. The dimensions are $n_{1} \times n_{2} \times n_{3}=38 \times 39 \times 40$. In the background, outside the helix, the tensors are fully isotropic with the eigenvalues having a magnitude of $10 \%$ of the maximal eigenvalue of the tensors within the helix. The exact generation details can be deciphered from our codes [21] written in Julia [3]. After generating the helix data, we took $s_{0}(\xi)=\|x(\xi)\|_{F}$. Then we generated $s_{k}(k=1, \ldots, 6)$ from the Stejskal-Tanner equation (6.1) with the diffusion-sensitising gradients $b_{1}=(1,0,0), b_{2}=(0,1,0), b_{3}=(0,0,1), b_{4}=(\sqrt{2}, \sqrt{2}, 0)$, $b_{5}=(\sqrt{2}, 0, \sqrt{2})$, and $b_{6}=(0, \sqrt{2}, \sqrt{2})$. To these diffusion-weighted images we added synthetic Gaussian noise of standard deviation $30 \%$ of the mean magnitude of $s_{0}$. As the regularisation parameter in the model (6.2), we took $\alpha=0.005$.

We only consider deterministic updates. We develop step length rules for Algorithm 4.1 based on Theorem 4.4, however, although $F_{\lambda}^{*}$ is strongly convex and the Moreau-Yosida regularisation makes also $F_{\mu, \gamma}^{*}$ strongly convex, we generally do not employ acceleration and instead keep the step length parameters fixed throughout the iterations. Therefore the theorem does not generally provide any convergence claims.

For convenience, we will identify the linear primal indices $j$ and dual indices $\ell$ (used for arbitrary blocks) with symbolic indices corresponding to the different variables $x, \mu, \lambda$ and their sub-blocks (used for specific blocks). The primal variable will be just a single block " $x$ " or be divided into voxelwise blocks " $x_{\xi}$ " for $\xi \in \Omega_{d}$. The dual variable will consist of just a single block " $y$ ", the two blocks corresponding to the variables " $\mu$ " and " $\lambda$ ", or " $\mu$ ", and the sub-blocks " $\lambda_{k, \xi}$ " over $k=1, \ldots, N$ and $\xi \in \Omega_{d}$.

Of the conditions of Theorem 4.4, we will not seek to satisfy the boundedness (4.7); following Theorem 3.13 this seems likely to hold if we initialise close enough to a solution and take the primal step length parameters $\stackrel{\circ}{j}_{j}^{0}$ small enough. However, we do not know, how small and how close they are required theoretically. Likewise, (4.6b), which with deterministic updates simplifies to $\delta \geq \stackrel{\circ}{\tau}_{j}^{0} \bar{L}$, is satisfied by taking $\stackrel{\circ}{\tau}_{j}^{0}$ small enough. To do this exactly, we would need to calculate the constant $L$ that satisfies the Lipschitz requirement of Assumption 3.1. Theorem 3.4 readily holds (with the Moreau-Yosida regularisation, as 
discussed above) with $\gamma_{G, x}=0$ and any $0 \leq \gamma_{F^{*}, \mu} \leq \gamma \alpha^{-1}$ and $0 \leq \gamma_{F^{*}, \lambda} \leq 1$. We take the latter as well as $\alpha_{y}$ and $\zeta_{\ell}$ such that (4.4) yields $\bar{\gamma}_{F^{*}, \ell} \equiv 0$ for all $\ell$. Theorem 3.2 we do not hope to verify in the confines of the present manuscript. With (4.6b) out of the way, for the calculation of the step lengths, it would only be needed for the constants $\gamma_{K, j}$. We simply make the reasonable assumption that we start close enough to a local minimiser satisfying the "second-order necessary condition" $\gamma_{G, j}+\gamma_{K, j} \geq 0$, i.e., $\gamma_{K, j} \geq 0$. Then we may simply assume $\gamma_{K, j}=0$ and are justified in taking $\widetilde{\gamma}_{G, j}=0$.

It remains to satisfy the relationship (4.6a) between the primal and dual step lengths. Taking the weights $w_{j, \ell, k}=w_{j, \ell, k}^{i}$ and the set of connections $\overline{\mathcal{V}}_{j}^{i}(\ell)=\overline{\mathcal{V}}_{j}(\ell)$ given in (4.3b) independent of the iteration and inserting $w_{j, k}$ from (4.3a) into (4.6a), the latter holds if

$$
1-\kappa \geq\left\|\sum_{j=1}^{m} \sqrt{\breve{\sigma}_{\ell}^{0} \dot{\tau}_{j}^{0} \chi_{\mathcal{V}_{j}^{i}}(\ell) \sum_{\ell^{\prime} \in \overline{\mathcal{V}}_{j}^{i}(\ell)} w_{j, \ell, \ell^{\prime}}} Q_{\ell} \nabla K\left(x^{i}\right) P_{j}\right\|^{2} .
$$

In particular, with just a single primal block $x$, we then satisfy (6.3) by taking

(6.4) $\breve{\sigma}_{\ell}^{0}=\frac{1-\kappa}{\tau_{x}^{0} \sum_{\ell^{\prime} \in \overline{\mathcal{V}}_{j}(\ell)} w_{x, \ell, \ell^{\prime}} R_{\ell}^{2}}, \quad$ where we need the estimate $\quad R_{\ell} \geq\left\|Q_{\ell} \nabla K\left(x^{i}\right)\right\|$.

Similarly to [5] we estimate $\left\|\mathcal{E}_{d}\right\| \leq R_{\mathcal{E}}:=\sqrt{12}$. Assuming that each $x^{i}(\xi)$ for $\xi \in \Omega_{d}$ is positive semi-definite, we also estimate with $r_{k, \xi}:=\left|s_{0}(\xi)\right|\left\|b_{k}\right\|_{2}^{2}$ that

$$
\left\|\nabla T\left(x^{i}\right)\right\| \leq R_{T}:=\sqrt{\sum_{k=1}^{N} \sum_{\xi \in \Omega_{d}} r_{k, \xi}^{2}} \quad \text { and } \quad\left\|\nabla K\left(x^{i}\right)\right\| \leq R:=\sqrt{R_{\mathcal{E}}^{2}+R_{T}^{2}} .
$$

We obtain $R_{\ell}$ for (6.4) from the same constituents $r_{k, \xi}$ and $R_{\mathcal{E}}$ depending on the exact block structure.

It then remains to choose the primal step lengths and the weights $w_{j, k, \ell}$. We consider the following four block structures and choices of weights:

(d1) As our reference case, corresponding to earlier non-block-adapted works [9, 33], a single primal block $x(m=1)$ and a single dual block $y(n=1)$. Based on the rough optimisation of the step length parameters illustrated in Figure 6.2a for a range of $\tau=i_{x}^{0}$ with $\breve{\sigma}_{y}^{0}=\sigma:=(1-\kappa) /\left(\tau R^{2}\right)$ with $\kappa=0.05$, we take $\tau:=1 / R$.

(d2) A single primal block $x(m=1)$ and the two dual blocks $\mu$ and $\lambda(n=2)$. We take $\tau=\leftarrow_{1}^{0}$ as in (d1) and with $w_{x, \lambda, \mu}:=R_{\mathcal{E}} /\left(R-R_{\mathcal{E}}\right)$ calculate from (6.4) the dual step length parameters as $\breve{\sigma}_{\mu}^{0}=(1-\kappa) /\left(\tau\left(1+w_{x, \lambda, \mu}^{-1}\right) R_{\mathcal{E}}^{2}\right)$ and $\breve{\sigma}_{\lambda}^{0}=(1-\kappa) /\left(\tau\left(1+w_{x, \lambda, \mu}\right) R_{T}^{2}\right)$. Thus $\breve{\sigma}_{\mu}^{0} R_{\mathcal{E}}$ equals $\sigma R$ of $(\mathrm{d} 1)$.

(d3) A single primal block $x(m=1)$ and in addition to the dual block $\mu$, we split $\lambda$ into voxelwise and $b_{k}$-wise blocks $\lambda_{k, \xi}\left(n=1+N n_{1} n_{2} n_{3}\right)$ indexed by $k=1, \ldots, N$ and $\xi \in \Omega_{d}$. We still take $\tau=\tau_{1}^{0}$ as in (d1) and with the setting $w_{x, \lambda_{(k, \xi)}, \mu}:=\sum_{k^{\prime}, \xi^{\prime}} r_{k^{\prime}, \xi^{\prime}} R_{\mathcal{E}} /\left(\left(R-R_{\mathcal{E}}\right) r_{k, \xi}\right)$ and $w_{x, \lambda_{(k, \xi)}, \lambda_{\left(k^{\prime}, \xi^{\prime}\right)}} \equiv 1$ calculate from (6.4) the dual step length parameters $\breve{\sigma}_{\mu}^{0}:=(1-\kappa) /\left(\tau\left(1+\sum_{k, \xi} w_{x, \lambda_{(k, \xi)}, \mu}^{-1}\right) R_{\mathcal{E}}^{2}\right)$ and $\breve{\sigma}_{\lambda_{k, \xi}}^{0}:=(1-\kappa) /\left(\tau\left(N+w_{x, \lambda_{(k, \xi)}, \mu}\right) r_{k, \xi}^{2}\right)$. This also keeps $\breve{\sigma}_{\mu}^{0} R_{\mathcal{E}}$ equal to $\sigma R$ of (d1).

(d4) Voxelwise primal blocks $x_{\xi}$ for $\xi \in \Omega\left(n=n_{1} n_{2} n_{3}\right)$ in addition to dual blocks as in (d3). We take the blockwise primal step length parameters according to $\tau_{\xi}^{0}=\tau_{\xi}:=R \tau /\left(1+N \max _{k=1, \ldots, N} r_{k, \xi}\right)$ for $\xi \in \Omega_{d}$, where $\tau$ is as in (d1). Then we take $w_{x_{\xi}, \lambda_{(k, \xi)}, \mu}:=r_{k, \xi}$ and $w_{x_{\xi}, \lambda_{(k, \xi)}, \lambda_{\left(k^{\prime}, \xi^{\prime}\right)}}=1$. Observe that by the definition 


\section{ETNA}

Kent State University and

Johann Radon Institute (RICAM)

of the connection set $\overline{\mathcal{V}}_{j}(\ell)$ in (4.3b), the dual block $(k, \xi)$ is not connected by $K$ to $\left(k^{\prime}, \xi^{\prime}\right)$ for $\xi^{\prime} \neq \xi$. By taking $\breve{\sigma}_{\mu}^{0}=(1-\kappa) /\left(\max _{\xi \in \Omega_{d}} \tau_{\xi}\left(1+\sum_{k=1}^{N} r_{k, \xi}\right) R_{\mathcal{E}}^{2}\right)$ and $\breve{\sigma}_{\lambda_{k, \xi}}^{0}=(1-\kappa) /\left(\tau_{\xi}\left(N+r_{k, \xi}^{-1}\right) r_{k, \xi}^{2}\right)$, we therefore satisfy (6.3). The maximum comes from estimating the norm in (6.3).

We report in Figure 6.2b for the first 10000 iterations the function value achieved by each algorithm variant. For (d3) and (d4) we also display the effect of the $O(1 / N)$-acceleration of Theorem 4.4; in (d1) and (d2) this has no notable effect.

On a mid-2014 MacBook Pro with a $2.8 \mathrm{GHz}$ Intel Core i5 processor and 16GB RAM running Julia 1.1.0, each iteration of (d1)-(d3) takes roughly 0.048 seconds. For (d4) this is roughly 0.062 seconds due to a more complicated primal update. ${ }^{1}$ However, in terms of computational times, (d4) is clearly much faster than the other variants: $0.77 \mathrm{~s}$ against 14.7-19.2s for (d1) and 13.6-18.1s for (d2) and (d3) to reach a function value 50. The time ranges account for us sampling the function values only every 100 iterations after the first 100. The visual character of the approximate solution provided by (d4) is on closer inspection slightly smoothed out compared to the other variants. This may be due to a non-optimal $\alpha$ in the model (6.2) or due to a different local solution.

6.2. Electrical impedance tomography. In this problem, we want to solve

$$
\min _{x \in V} \sum_{k=1}^{N} \frac{1}{2}\left\|A_{k}(x)\right\|^{2}+\alpha\|\nabla x\|_{2,1}
$$

on a finite-dimensional subspace $V \subset L^{2}(\Omega)$ with $\Omega \subset \mathbb{R}^{2}$ and each $A_{k}: V \rightarrow \mathbb{R}^{N}$ a nonlinear operator corresponding to the fit of the solution of a partial differential equation controlled by $x$ to the measured data. We specifically use the complete electrode model of EIT [41]. Our implementation of the model will be described in detail in [18]. The rough idea is that $N$ electrodes are placed on the boundary of the domain $\Omega$ inside which we want to reconstruct an unknown conductivity $x$; see Figure 6.3, which presents a synthetic 2D slice model of an object in a cylindrical water tank. As our data, we only have $N$ boundary measurements corresponding to exciting in turn each of the electrodes $k=1, \ldots, N$ with a positive electric potential. In each of these excitations, the remaining electrodes are grounded, and the electric current generated by these excitations is measured at each electrode, yielding $N$ measurements. The operators $A_{k}$ correspond to each such excitation setup. In the example of Figure 6.3, the number of electrodes $N=16$.

We can again write this problem in the form $(\mathrm{S})$ with

$$
G(x):=0, \quad K(x):=\left(\nabla x, A_{1}(x), \ldots, A_{N}(x)\right), \quad \text { and } \quad F^{*}(y)=\delta_{\alpha \mathbb{B}}(\mu)+\sum_{k=1}^{N}\left\|\lambda_{k}\right\|_{2}^{2},
$$

where $y=\left(\mu, \lambda_{1}, \ldots, \lambda_{N}\right)$ and $\mathbb{B}$ is the product of the pointwise Euclidean unit balls of $\mathbb{R}^{2}$ over $\Omega$.

As a first case of the dual blocks, we take $y_{0}$ corresponding to the total variation term, and the full measurement vectors $y_{k}$ corresponding to each excitation $k=1, \ldots, N$. We estimate $\|\nabla\| \leq R_{\nabla}$ for $R_{\nabla}$ being the largest singular value of $\nabla$ on $V$. We do not have exact

\footnotetext{
${ }^{1}$ In the Julia code [21], we update $x^{i+1}(\xi):=x^{i}(\xi)-\tau_{\xi} \Delta x^{i}(\xi)$ and $\lambda^{i+1}(k, \xi):=\left(\lambda^{i}(k, \xi)+\right.$ $\left.\sigma_{k, \xi} \Delta \lambda^{i}(k, \xi)\right) /\left(1+\sigma_{k, \xi}\right)$ for some temporary $\Delta x^{i}$ and $\Delta \lambda^{i}$ and all $\xi \in \Omega_{d}$ and $k=1, \ldots, N$. The latter does not appear to cause a notable performance penalty compared to a spatially constant $\sigma$ while the former does. However, each $x^{i+1}(\xi)$ is a tensor consisting of multiple floating point numbers while $\lambda^{i+1}(k, \xi)$ is a single floating point number. Our guess is that, due to uneven memory indexing when $\tau$ is spatially varying, the tensor update cannot make as good use of the processor SIMD instructions.
} 


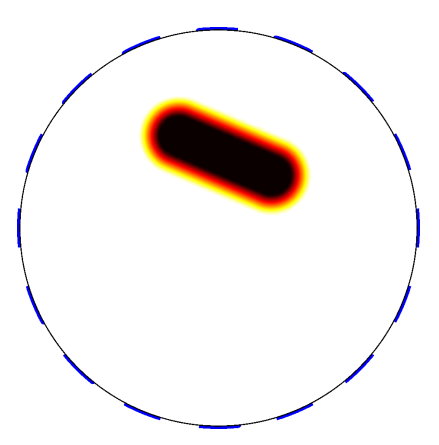

(a) Synthetic conductivity.

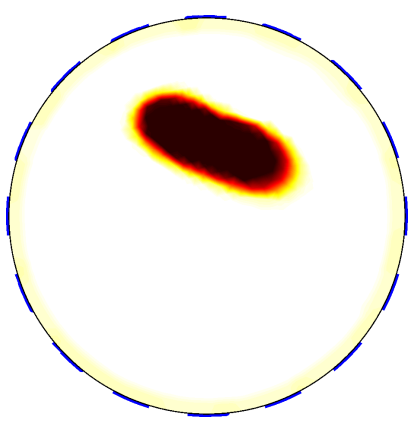

(b) Reconstructed conductivity.

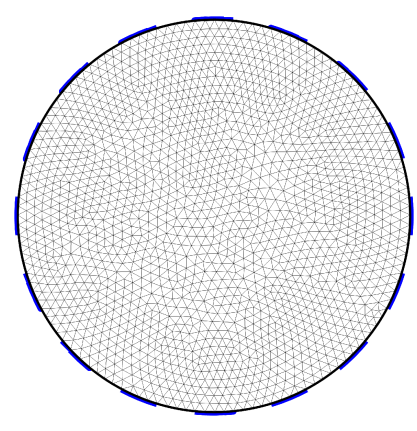

(c) Finite element mesh.

FIG. 6.3. Synthetic true conductivity and reconstructed conductivity for the EIT example. The reconstruction is the one obtained with the block structure and dual step length setup of (e3) with $\tau=500 / R$ after 15000 iterations. The blue patches on the boundary of the domain indicate the electrodes. We display in (c) the finite element mesh used to represent the conductivity.

estimates for the norm of $\nabla A_{k}\left(x^{i}\right)$. Therefore, we take a dynamic norm estimate $r_{k}=r_{k}(i)$ over the last 100 iterations,

$$
\left\|\nabla A_{k}\left(x^{i}\right)\right\| \leq r_{k}:=1.05 \max _{\max \{i-99,0\} \leq \iota \leq i}\left\|\nabla A_{k}\left(x^{\iota}\right)\right\| \quad(k=1, \ldots, N) .
$$

We may then estimate $\left\|\nabla K\left(x^{i}\right)\right\| \leq R:=\sqrt{R_{\nabla}^{2}+r_{1}^{2}+\cdots+r_{N}^{2}}$. As a second case, we further split each $y_{k}$ into sub-blocks $y_{k, j} \in \mathbb{R}$ corresponding to each individual electrode $j=1, \ldots, N$ being measured. We then take norm estimates $r_{k, j}=r_{k, j}(i)$ over the last 100 iterations,

$$
\left|\left[\nabla A_{k}\left(x^{i}\right)\right]_{j}\right| \leq r_{k, j}:=1.05 \max _{\max \{i-99,0\} \leq \iota \leq i}\left|\left[\nabla A_{k}\left(x^{\iota}\right)\right]_{j}\right| \quad(k, j=1, \ldots, N) .
$$

We work in the setting of Section 5. Note that unlike Algorithm 4.1 in the DTI experiments of Section 6.1, Algorithm 5.1 allows partial calculation of $K$ in both the primal and dual updates, which should in principle be beneficial in stochastic methods. We develop step length rules for Algorithm 5.1 based on Theorem 5.3. Similarly to (6.4), with $w_{j, \ell, k}=w_{j, \ell, k}^{i}$ and $\overline{\mathcal{V}}_{j}^{i}(\ell)=\overline{\mathcal{V}}_{j}(\ell)$ independent of the iteration, for non-stochastic methods with a single primal block $x,(5.6 a)$ in particular holds by taking

$$
\stackrel{\circ}{\sigma}_{\ell}^{1}=\frac{1-\kappa}{\breve{\tau}_{x}^{0} \sum_{\ell^{\prime} \in \overline{\mathcal{V}}_{j}(\ell)} w_{x, \ell, \ell^{\prime}} R_{\ell}^{2}}, \quad \text { where we estimate } \quad R_{\ell} \geq\left\|Q_{\ell} \nabla K\left(x^{i}\right)\right\|
$$

Again, for convenience, we identify the linear primal indices $j$ and dual indices $\ell$ and $\ell^{\prime}$ with symbolic indices $x, \mu$, and $\lambda_{k}$. It then remains to choose $\breve{\tau}_{x}^{0}$ and the weights $w_{x, \ell, \ell^{\prime}}$. For this we consider four different block and weight setups:

(e1) Again, as our reference case, corresponding to earlier non-block-adapted works $[9,33]$, a single primal block $x(m=1)$ and a single dual block $y(n=1)$. Based on rough optimisation of the step length parameters, illustrated in Figure 6.4a for a range of $\tau=\breve{\tau}_{x}^{0}$ with $\stackrel{\circ}{\sigma}_{y}^{1}=(1-\kappa) /\left(\tau R^{2}\right)$ with $\kappa=0.05$, we take $\tau:=5 / R$ for $R$ computed using just the initial iterate $x^{0}$ as explained above.

(e2) A single primal block $x(m=1)$ and the dual blocks $\mu, \lambda_{1}, \ldots, \lambda_{N}$. We take $\tau=\breve{\tau}_{x}^{0}$ as in (e1) and with $w_{x, \lambda_{p}, \mu}:=\sum_{k} r_{k} R_{\nabla} /\left(\left(R-R_{\nabla}\right) r_{p}\right)$ and $w_{x, \lambda_{p}, \lambda_{k}}:=1$ for $p, k=1, \ldots, N$, solve from (6.6) that $\circ_{\mu}^{1}:=(1-\kappa) /\left(\tau\left(1+\sum_{k} w_{x, \lambda_{k}, \mu}^{-1}\right) R_{\nabla}^{2}\right)$ and 


\section{ETNA}

Kent State University and

Johann Radon Institute (RICAM)

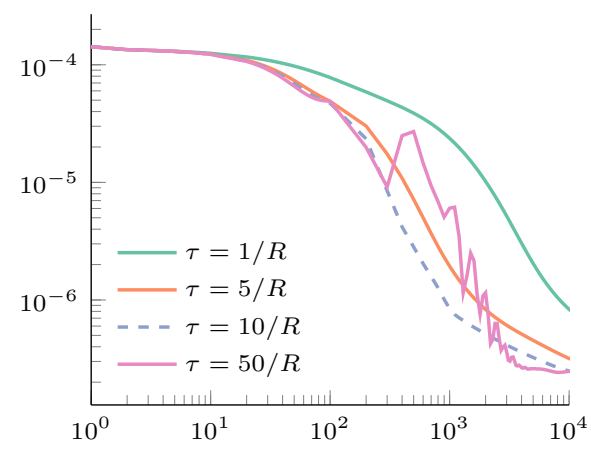

(a) Reference algorithm (e1), multiple step lengths.

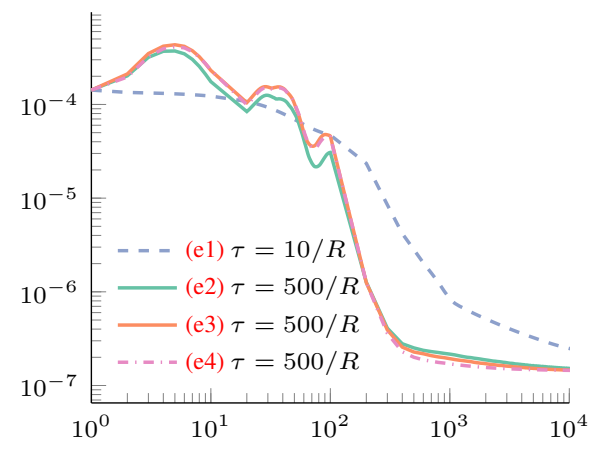

(b) Comparison of algorithm variants (e1)-(e4).

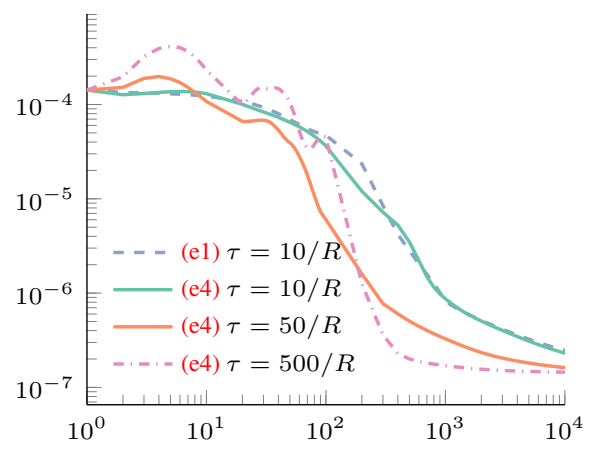

(c) Blocked algorithm (e4), multiple step lengths.

FIG. 6.4. EIT reconstruction performance: iteration counts are on the $x$ axis and primal objective function values (6.5) are on the $y$ axis. We start with step length justification for the non-blocked reference algorithm (e1) in (a). Based on this we use a step length $\tau=10 / R$ for the reference algorithm as higher step lengths become unstable. Comparison of the different blocked algorithm variants is given in (b) for $\tau=500 / R$ : with lower parameters the differences are less noticeable, and with higher parameters insignificant improvement is obtained. Based on this, in (c) we represent the performance of (e4) for multiple step lengths.

$\stackrel{\circ}{\sigma}_{\lambda_{p}}^{1}:=(1-\kappa) /\left(\tau\left(N+w_{x, \lambda_{p}, \mu}\right) r_{p}^{2}\right)$ for $p=1, \ldots, N$. This case and the step length rules are analogous to (d3) for DTI.

(e3) As (e2) but split each $\lambda_{p}$ into further measurement-wise dual blocks $y_{p, j}$ $(p, j=1, \ldots, N)$, replacing in the expressions of (e2) the indices $p$ and $k$ by $(p, j)$ and $\left(k, j^{\prime}\right)$ with $j, j^{\prime} \in\{1, \ldots, N\}$. Thus $r_{k}$ becomes $r_{k, j^{\prime}}$, etc.

(e4) Measurement-wise dual blocks as in (e3) but $w_{x, \lambda}(p, j), \mu:=r_{p, j}^{-1}$.

The performance of the algorithm variants (e1)-(e4) is depicted in Figure 6.4 with a sample reconstruction in Figure 6.3b. Observe how the block-adapted algorithms allow in practise larger $\tau$ than the reference algorithm without block-adaptation. This has significant performance benefits: To reach and stay below the objective function value of the order $10^{-7}$, (e4) with $\tau=500 / R$ requires 208 iterations while (e1) with $\tau=10 / R$ requires 906 iterations. (With $\tau=500 / R$ the latter requires 3544 iterations, no longer converging well with high $\tau$.) We also tested stochastic variants of the algorithms for the EIT problem, updating in each iteration only a random subset of the dual blocks. This did not, however, offer any performance benefits over the block-adapted variants, neither in terms of epoch count (iteration count scaled by the fraction of updated blocks) nor actual computational time. 


\section{ETNA}

Kent State University and

Johann Radon Institute (RICAM)

7. Conclusion. In this paper, we studied block-proximal primal-dual splitting methods for non-convex non-smooth optimisation. From an abstract starting point-also able to model doubly-stochastic methods-we derived explicit algorithms and step-length bounds for two particular cases: methods with full dual updates and methods with full primal updates. For both cases, we derived rules ensuring local $O(1 / N), O\left(1 / N^{2}\right)$, and linear rates under varying conditions and choices of the step lengths parameters.

We demonstrated the performance of the methods on practical inverse problems. Based on our experience with both the DTI and EIT examples, the block-adaptation provides significant performance benefits. Random updates, by contrast, did not offer benefits in our sample problems. We suspect that they might be more beneficial for very large scale problems that do not share work between the blocks, yet where the blocks have overlapping information, or where communication delays within a computing cluster become significant. This may be one of the possible directions for further research on the presented methods and their application.

Acknowledgements. This work was started when T. Valkonen and S. Mazurenko were at the University of Liverpool. There their work was supported by the EPSRC First Grant EP/P021298/1. More recently, T. Valkonen has been supported by the Academy of Finland grants 314701 and 320022 as well as the Escuela Politécnica Nacional internal grant PIJ-18-03. S. Mazurenko has been supported by the Operational Programme Research, Development and Education project MSCAfellow@MUNI (CZ.02.2.69/0.0/0.0/17_050/0008496). J. Jauhiainen has been supported by the European Union Horizon 2020 research and innovation programme under grant agreement No 764810.

A data statement for the EPSRC. The codes and data for the DTI experiments are available at [21]. The codes for EIT, based on historical work of several people, cannot be made available at this point.

\section{Appendix A. Satisfaction of the three-point condition.}

The following lemma provides simplified conditions under which Assumption 3.2 holds, e.g., whenever $x \mapsto\langle K(x), \widehat{y}\rangle$ is block-separable and strongly-convex.

LEMmA A.1. Suppose that Assumption 3.1 holds and the following is true for the given neighbourhood $\mathcal{X}_{K}$ of $\widehat{x}, \Gamma_{K}=\sum_{j=1}^{m} \gamma_{K, j} P_{j} \in \mathbb{L}(X ; X), \gamma_{K, j} \in \mathbb{R}$, some $\gamma_{x}>0$ :

$$
\begin{gathered}
\left\langle\left[\nabla K\left(x^{\prime}\right)-\nabla K(\widehat{x})\right]^{*} \widehat{y}, x^{\prime}-\widehat{x}\right\rangle \\
\geq\left\|x^{\prime}-\widehat{x}\right\|_{\Gamma_{K}}^{2}+\gamma_{x}\left\|x^{\prime}-\widehat{x}\right\|^{2}, \\
\left\langle\left[P_{j} \nabla K\left(x^{\prime}\right)-P_{j} \nabla K(\widehat{x})\right]^{*} \widehat{y}, x_{j}^{\prime}-\widehat{x}_{j}\right\rangle \geq \gamma_{K, j}\left\|x_{j}^{\prime}-\widehat{x}_{j}\right\|^{2} \quad(j=1, \ldots, m) .
\end{gathered}
$$

Let $\beta_{1}, \beta_{2}>0, A=\sum_{j=1}^{m} a_{j} P_{j}$, and $\underline{a}:=\min _{j} a_{j}$. Then Assumption 3.2 holds for $p=1$ when

$$
\begin{aligned}
L \theta_{A} & \leq \underline{a}\left(\gamma_{x}-\beta_{1}\right)-\beta_{2} \max _{j}\left(a_{j}-\underline{a}\right) \quad \text { and } \\
L_{3} & \geq L^{2}\left\|P_{\mathrm{NL}} \widehat{y}\right\|\left(\beta_{1}^{-1}+\left(\beta_{2} \underline{a}\right)^{-1} \sum_{j=1}^{m}\left(a_{j}-\underline{a}\right)\right) / 2+2 L \theta_{A} .
\end{aligned}
$$

Proof. We need to study (3.2). We have

$$
\begin{aligned}
R^{K}:= & \left\langle[\nabla K(x)-\nabla K(\widehat{x})]^{*} \widehat{y}, x^{\prime}-\widehat{x}\right\rangle_{A}-\left\|x^{\prime}-\widehat{x}\right\|_{A \Gamma_{K}}^{2} \\
=\underline{a} & \left(\left\langle[\nabla K(x)-\nabla K(\widehat{x})]^{*} \widehat{y}, x^{\prime}-\widehat{x}\right\rangle-\left\|x^{\prime}-\widehat{x}\right\|_{\Gamma_{K}}^{2}\right) \\
& \quad+\sum_{j=1}^{m}\left(a_{j}-\underline{a}\right)\left(\left\langle[\nabla K(x)-\nabla K(\widehat{x})]^{*} \widehat{y}, x_{j}^{\prime}-\widehat{x}_{j}\right\rangle-\gamma_{K, j}\left\|x_{j}^{\prime}-\widehat{x}_{j}\right\|^{2}\right) .
\end{aligned}
$$


We now apply (A.1a), Young's inequality with the factor $\beta_{1}>0$, and Assumption 3.1 to bound

$$
\begin{aligned}
\langle[\nabla K(x)- & \left.\nabla K(\widehat{x})]^{*} \widehat{y}, x^{\prime}-\widehat{x}\right\rangle-\left\|x^{\prime}-\widehat{x}\right\|_{\Gamma_{K}}^{2} \\
= & \left\langle\left[\nabla K\left(x^{\prime}\right)-\nabla K(\widehat{x})\right]^{*} \widehat{y}, x^{\prime}-\widehat{x}\right\rangle-\left\|x^{\prime}-\widehat{x}\right\|_{\Gamma_{K}}^{2} \\
& \quad+\left\langle\left[\nabla K(x)-\nabla K\left(x^{\prime}\right)\right]^{*} \widehat{y}, x^{\prime}-\widehat{x}\right\rangle \\
\geq & \left(\gamma_{x}-\beta_{1}\right)\left\|x^{\prime}-\widehat{x}\right\|^{2}-L^{2}\left\|P_{\mathrm{NL}} \widehat{y}\right\|^{2}\left(4 \beta_{1}\right)^{-1}\left\|x^{\prime}-x\right\|^{2}
\end{aligned}
$$

Similarly, for any $\beta_{2}>0$, we have

$$
\begin{aligned}
\langle[\nabla K(x)- & \left.\nabla K(\widehat{x})]^{*} \widehat{y}, x_{j}^{\prime}-\widehat{x}_{j}\right\rangle \\
= & \left\langle\left[P_{j} \nabla K\left(x^{\prime}\right)-P_{j} \nabla K(\widehat{x})\right]^{*} \widehat{y}, x_{j}^{\prime}-\widehat{x}_{j}\right\rangle \\
& \quad+\left\langle\left[\nabla K(x)-\nabla K\left(x^{\prime}\right)\right]^{*} \widehat{y}, x_{j}^{\prime}-\widehat{x}_{j}\right\rangle \\
\geq & \gamma_{K, j}\left\|x_{j}^{\prime}-\widehat{x}_{j}\right\|^{2}-L^{2}\left\|P_{\mathrm{NL}} \widehat{y}\right\|^{2}\left(4 \beta_{2}\right)^{-1}\left\|x^{\prime}-x\right\|^{2}-\beta_{2}\left\|x_{j}^{\prime}-\widehat{x}_{j}\right\|^{2} .
\end{aligned}
$$

Combining the two estimates, we arrive at

$$
\begin{aligned}
R^{K} \geq & \underline{a}\left(\gamma_{x}-\beta_{1}\right)\left\|x^{\prime}-\widehat{x}\right\|^{2}-\underline{a} L^{2}\left\|P_{\mathrm{NL}} \widehat{y}\right\|^{2}\left(4 \beta_{1}\right)^{-1}\left\|x^{\prime}-x\right\|^{2} \\
& \quad-\sum_{j=1}^{m}\left(a_{j}-\underline{a}\right)\left(\beta_{2}\left\|x_{j}^{\prime}-\widehat{x}_{j}\right\|+L^{2}\left\|P_{\mathrm{NL}} \widehat{y}\right\|^{2}\left\|x^{\prime}-x\right\|^{2}\right) \\
= & \sum_{j=1}^{m}\left(\underline{a}\left(\gamma_{x}-\beta_{1}\right)-\left(a_{j}-\underline{a}\right) \beta_{2}\right)\left\|x_{j}^{\prime}-\widehat{x}_{j}\right\|^{2} \\
& \quad-\underline{a} L^{2}\left\|P_{\mathrm{NL}} \widehat{y}\right\|\left(\beta_{1}^{-1}+\left(\beta_{2} \underline{a}\right)^{-1} \sum_{j=1}^{m}\left(a_{j}-\underline{a}\right)\right)\left\|x^{\prime}-x\right\|^{2} / 4 .
\end{aligned}
$$

At the same time, using Assumption 3.1, we get for the right-hand side of (3.2) the bound

$$
\|K(\widehat{x})-K(x)-\nabla K(x)(\widehat{x}-x)\| \leq \frac{L}{2}\|x-\widehat{x}\|^{2} \leq L\left\|x^{\prime}-\widehat{x}\right\|^{2}+L\left\|x^{\prime}-x\right\|^{2} .
$$

Hence, Assumption 3.2 holds if we take $p=1, L \theta_{A} \leq \min _{j} \underline{a}\left(\gamma_{x}-\beta_{1}\right)-\left(a_{j}-\underline{a}\right) \beta_{2}$, and $L_{3} \geq L^{2}\left\|P_{\mathrm{NL}} \widehat{y}\right\|\left(\beta_{1}^{-1}+\left(\beta_{2} \underline{a}\right)^{-1} \sum_{j=1}^{m}\left(a_{j}-\underline{a}\right)\right) / 2+2 L \theta_{A}$.

\section{Appendix B. A technical lemma.}

LemmA B.1. We have $\bar{z}_{N} \leq \bar{z}_{0}+N / 2$ whenever $z_{j}^{i}>0(i=1, \ldots, N ; j=1, \ldots, m)$ satisfy

$$
z_{j}^{i+1}=\frac{1+z_{j}^{i}}{\sqrt{1+\bar{z}_{i}^{-1}}} \text { with } \quad \bar{z}_{i}:=\max _{j=1, \ldots, m} z_{j}^{i} .
$$

Proof. Taking $\max _{j=1, \ldots, m}$ on both sides of the first part of (B.1), we obtain

$$
\bar{z}_{i+1}=\left(1+\bar{z}_{i}\right) \sqrt{\frac{\bar{z}_{i}}{\bar{z}_{i}+1}}=\sqrt{\bar{z}_{i}^{2}+\bar{z}_{i}}
$$

We thus obtain the claim by telescoping

$$
\bar{z}_{i+1}-\bar{z}_{i}=\sqrt{\bar{z}_{i}^{2}+\bar{z}_{i}}-\bar{z}_{i}=\frac{\bar{z}_{i}}{\sqrt{\bar{z}_{i}^{2}+\bar{z}_{i}}+\bar{z}_{i}}=\frac{1}{\sqrt{1+\bar{z}_{i}^{-1}}+1} \leq \frac{1}{2} .
$$

This finishes the proof. 


\section{ETNA}

Kent State University and

Johann Radon Institute (RICAM)

\section{REFERENCES}

[1] P. J. BASSER AND D. K. JONES, Diffusion-tensor MRI: theory, experimental design and data analysis - a technical review, NMR Biomed., 15 (2002), pp. 456-467.

[2] D. P. BERTSEKAS, Incremental aggregated proximal and augmented Lagrangian algorithms, Preprint on arXiv, 2015. https: / / arxiv.org/abs/1509.09257

[3] J. Bezanson, A. Edelman, S. KARPinski, And V. B. ShaH, Julia: a fresh approach to numerical computing, SIAM Rev., 59 (2017), pp. 65-98.

[4] P. BiAnCHI, W. HACHEM, AND I. FRANCK, A stochastic coordinate descent primal-dual algorithm and applications, in 2014 IEEE International Workshop on Machine Learning for Signal Processing (MLSP), IEEE Conference Proceedings, Los Alamitos, 2014, 6 pages.

[5] A. Chambolle, An algorithm for total variation minimization and applications, J. Math. Imaging Vision, 20 (2004), pp. 89-97.

[6] A. Chambolle, M. J. Ehrhardt, P. Richtárik, And C.-B. Schönlieb, Stochastic primal-dual hybrid gradient algorithm with arbitrary sampling and imaging applications, SIAM J. Optim., 28 (2018), pp. 2783-2808.

[7] A. CHAMBOLle AND T. POCK, A first-order primal-dual algorithm for convex problems with applications to imaging, J. Math. Imaging Vision, 40 (2011), pp. 120-145.

[8] R. CHAN, H. YANG, AND T. ZENG, A two-stage image segmentation method for blurry images with Poisson or multiplicative gamma noise, SIAM J. Imaging Sci., 7 (2014), pp. 98-127.

[9] C. Clason, S. Mazurenko, and T. Valkonen, Acceleration and global convergence of a first-order primal-dual method for nonconvex problems, SIAM J. Optim., 29 (2019), pp. 933-963.

[10] - Primal-dual proximal splitting and generalized conjugation in nonsmooth nonconvex optimization, Appl. Math. Optim, (2020). https://doi.org/10.1007/s00245-020-09676-1.

[11] C. Clason AND T. VAlKonen, Primal-dual extragradient methods for nonlinear nonsmooth PDEconstrained optimization, SIAM J. Optim., 27 (2017), pp. 1314-1339.

[12] P. L. COMBETTES AND J. PESQUET, Stochastic forward-backward and primal-dual approximation algorithms with application to online image restoration, in 2016 24th European Signal Processing Conference (EUSIPCO), IEEE Conference Proceedings, Los Alamitos, 2016, pp. 1813-1817.

[13] D. CSIBA, Z. QU, AND P. RICHTTÁRIK, Stochastic dual coordinate ascent with adaptive probabilities, in Proceedings of the 32nd International Conference on Machine Learning, F. Bach and D. Blei, eds., vol. 37 of Proceedings of Machine Learning Research, PMLR, 2015, pp. 674-683.

[14] D. DAVIS AND D. DRUSVYATSKIY, Stochastic model-based minimization of weakly convex functions, SIAM J. Optim., 29 (2019), pp. 207-239.

[15] O. FERCOQ AND P. BIANCHI, A coordinate-descent primal-dual algorithm with large step size and possibly nonseparable functions, SIAM J. Optim., 29 (2019), pp. 100-134.

[16] O. FERCOQ AND P. RICHTÁRIK, Accelerated, parallel, and proximal coordinate descent, SIAM J. Optim., 25 (2015), pp. 1997-2023.

[17] P. Getreuer, M. TONG, AND L. A. Vese, A variational model for the restoration of MR images corrupted by blur and Rician noise, in Advances in Visual Computing, G. Bebis et al., eds., vol. 6938 of Lecture Notes in Computer Science, Springer, Berlin, 2011, pp. 686-698.

[18] J. Jauhiainen, P. Kuusela, A. Seppënen, And T. VAlKonen, Relaxed Gauss-Newton methods with applications to electrical impedance tomography, SIAM J. Imaging Sci., 13 (2020), pp. 1415-1445.

[19] P. KINGSLEY, Introduction to diffusion tensor imaging mathematics: Parts I-III, Concepts Magn. Res. Part A, 28 (2006), pp. 101-179.

[20] A. Martín AND E. SCHIAVI, Automatic total generalized variation based DTI Rician denoising, Int. Conf. in Image Analysis and Recognition, M. Kamel and A. Campilho, eds., vol. 7950 of Lecture Notes in Computer Science, Springer, Heidelberg, 2013, pp. 581-588.

[21] S. Mazurenko, J. Jauhiainen, And T. VAlkonen, Diffusion tensor imaging codes from "primal-dual block-proximal splitting for a class of non-convex problems”. Software on Zenodo, November 2019. doi:10.5281/zenodo.3528137

[22] A. Milzarek, X. Xiao, S. Cen, Z. Wen, And M. Ulbrich, A stochastic semismooth Newton method for nonsmooth nonconvex optimization, SIAM J. Optim., 29 (2019), pp. 2916-2948.

[23] Y. NESTEROV, Efficiency of coordinate descent methods on huge-scale optimization problems, SIAM J. Optim., 22 (2012), pp. 341-362.

[24] D. Nishimura, Principles of Magnetic Resonance Imaging, Stanford University, Stanford, 1996.

[25] Z. PENG, Y. XU, M. YAN, AND W. YIN, ARock: an algorithmic framework for asynchronous parallel coordinate updates, SIAM J. Sci. Comput., 38 (2016), pp. A2851-A2879.

[26] M. PILANCI AND M. J. WAINWRIGHT, Iterative Hessian sketch: fast and accurate solution approximation for constrained least-squares, J. Mach. Learn. Res., 17 (2016), Paper No. 53, 38 pages.

[27] Z. QU, P. RICHTÁRIK, M. TAKÁČ, AND O. FERCOQ, SDNA: Stochastic dual newton ascent for empirical risk minimization, in Proceedings of The 33rd International Conference on Machine Learning, M. F. Balcan 
and K. Q. Weinberger, eds., vol. 48 of Proceedings of Machine Learning Research, PMLR, 2016, pp. $1823-$ 1832.

[28] P. RichtÁRIK AND M. TAKÁČ, Distributed coordinate descent method for learning with big data, J. Mach. Learn. Res., 17 (2016), Paper No. 75, 25 pages.

[29] _ _ Parallel coordinate descent methods for big data optimization, Math. Program., 156 (2016), pp. 433484.

[30] R. T. Rockafellar And R. J.-B. Wets, Variational Analysis, Springer, Berlin, 1998.

[31] S. SHALEV-SHWARTZ AND T. ZHANG, Accelerated proximal stochastic dual coordinate ascent for regularized loss minimization, Math. Program., 155 (2016), pp. 105-145.

[32] T. SUZUKI, Stochastic dual coordinate ascent with alternating direction method of multipliers, in Proceedings of the 31st International Conference on Machine Learning, E. P. Xing and T. Jebara, eds., vol. 32 of Proceedings of Machine Learning Research, PMLR, 2014, pp. 736-744.

[33] T. VALKONEN, A primal-dual hybrid gradient method for non-linear operators with applications to MRI, Inverse Problems, 30 (2014), Art. 055012, 45 pages.

[34] _ Testing and non-linear preconditioning of the proximal point method, Appl. Math. Optim., (2018). https://doi.org/10.1007/s00245-018-9541-6

[35] _ _ Block-proximal methods with spatially adapted acceleration, Electron. Trans. Numer. Anal., 51 (2019), pp. 15-49. http://etna.ricam.oeaw.ac.at/vol.51.2019/pp15-49.dir/pp15-49.pdf

[36] - First-order primal-dual methods for nonsmooth nonconvex optimisation, in Handbook of Mathematical Models and Algorithms in Computer Vision and Imaging, K. Chen, C.-B. Schönlieb, X.-C. Tai, and L. Younes, eds., Springer, 2020, in press.

[37] T. VAlKONEN, K. BREDIES, AND F. KNOLL, TGV for diffusion tensors: a comparison of fidelity functions, J. Inverse Ill-Posed Probl., 21 (2013), pp. 355-377.

[38] - Total generalised variation in diffusion tensor imaging, SIAM J. Imaging Sci. 6 (2013), pp. 487-525.

[39] T. VALKONEN AND M. LiEBMANN, GPU-accelerated regularisation of large diffusion tensor volumes, Computing, 95 (2013), pp. S771-S784.

[40] VARIOUS AUTHORS, Teem toolkit, version 1.11.0, 2012. http: / / teem. source forge. net.

[41] P. J. VauhKonen, M. VauhKonen, T. SaVolainen, and J. P. Kaipio, Three-dimensional electrical impedance tomography based on the complete electrode model, IEEE Trans. Biomed. Engrg., 46 (1999), pp. $1150-1160$

[42] S. J. WRIGHT, Coordinate descent algorithms, Math. Program., 151 (2015), pp. 3-34.

[43] Y. XU AND W. YIN, Block stochastic gradient iteration for convex and nonconvex optimization, SIAM J. Optim., 25 (2015), pp. 1686-1716.

[44] _ A globally convergent algorithm for nonconvex optimization based on block coordinate update, J. Sci. Comput., 72 (2017), pp. 700-734.

[45] Y. ZHANG AND L. XIAO, Stochastic primal-dual coordinate method for regularized empirical risk minimization, J. Mach. Learn. Res., 18 (2017), Paper No. 84, 42 pages.

[46] P. ZHAO AND T. ZHANG, Stochastic optimization with importance sampling for regularized loss minimization, in Proceedings of the 32nd International Conference on Machine Learning, F. Bach and D. Blei, eds., vol. 37 of Proceedings of Machine Learning Research, 2015, PMLR, pp. 1-9. 